\title{
The First Spectrum of Ytterbium (Yb I)
}

\author{
William F. Meggers* and Jack L. Tech \\ Institute for Basic Standards, National Bureau of Standards, Washington, D.C. 20234
}

\begin{abstract}
(September 15, 1977)
Wavelengths and estimated intensities for 1791 lines of neutral ytterbium in the region 2155 to $31308 \AA$ are reported. The Zeeman effect has been investigated for 249 of these lines. Analysis of the data has resulted in classification of 787 lines as transitions between 102 even and 77 odd levels. Even levels have been assigned to the electron configurations $4 f^{14} 6 s^{2}$ (the ground state), $4 f^{14} 6 s n s, 4 f^{14} 6 s n d, 4 f^{13} 6 s^{2} 6 p, 4 f^{14} 6 p^{2}, 4 f^{14} 5 d^{2}$, and $4 f^{13} 5 d 6 s 6 p$. Odd levels have been assigned to $4 f^{14} 6 s n p, 4 f^{14} 6 s n f$, and $4 f^{13} 5 d 6 s^{2}$. Many odd levels are still unassigned, but the majority undoubtedly belong to the configuration $4 f^{13} 5 d^{2} 6 s$.
\end{abstract}

Key words: Energy Levels, Yb I; spectrum, Yb I; wavelengths, Yb I; Yb I; ytterbium; Zeeman effect.

\section{Introduction}

The first regularities in $\mathrm{Yb}$ I were reported by Meggers and Scribner [1937 $]^{1}$ in their description of the conventional are and spark spectra of this element in the range 2000 to $11000 \AA$. In that paper they reported some 400 lines of $\mathrm{Yb}$ I observed by burning $\mathrm{Yb}$ salt in an arc. Their assignment of lines of successive spectra agrees almost perfectly with King's [1931] separation of ionization stages based upon a study of the spectra of ytterbium excited in an electricfurnace. Their first measurements of $\mathrm{Yb}$ spectra were, however, made in 1929 when the investigation of Lu spectra was begun. Since all available samples of lutecium at that time were contaminated with ytterbium, it was necessary to study $\mathrm{Yb}$ spectra simultaneously in order to derive trustworthy descriptions of Lu spectra. Meggers thus carried on the work intermittently for a period spanning 37 years until his death in 1966. He left most of the analysis presented here.

Most of the $\mathrm{Yb}$ I lines used in the present analysis were published in the general description of ytterbium spectra by Meggers and Corliss [1966]. That list extends from 2155 to $11603 \AA$ and includes wavelengths, estimated intensities as observed with a "Meggers lamp" and with a "Thomson lamp," and the Zeeman type. A typical spectrogram from which the wavelengths were measured is shown in figure 1 .

Humphreys and Paul [1959] extended the observations to $24552 \AA$ with an electrodeless lamp as source, and in 1964 they reported three more lines at still longer wavelengths, reaching $31308 \AA$ (unpublished).

Sugar [1962] developed a pulsed-are light source for producing self-reversed lines in the first and second spectra of rare-earth elements. The first observations he made with this source were with ytterbium electrodes and covered the range 2400 to $7000 \AA$. These spectrograms were evaluated by Meggers, who found that 22 lines of $\mathrm{Yb}$ I were absorbed or self-reversed.

In 1963 N. Spector photographed the spectra of ytterbium emitted by a sliding spark in the region from 6600 to

\footnotetext{
* Deceased.

${ }^{1}$ Years in brackets indicate the literature references at the end of this paper.
}
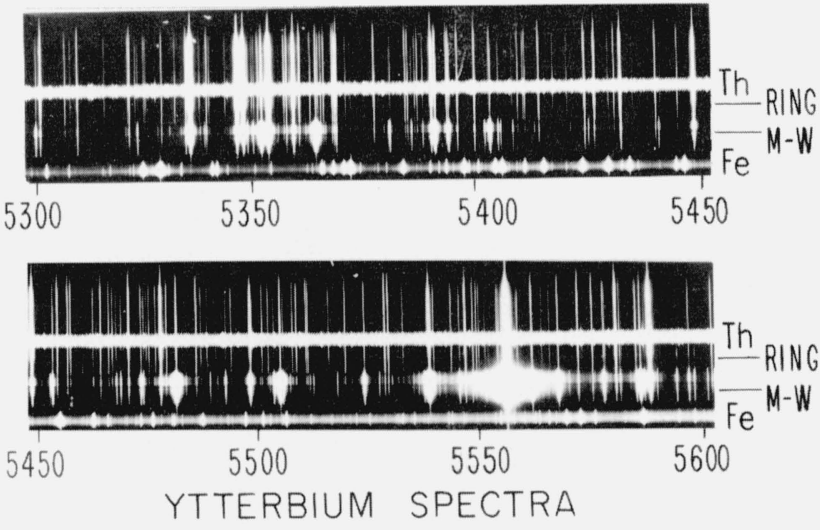

FiguRE 1. Typical spectrogram of ytterbium in the green region of the spectrum.

$11600 \AA$, and Meggers found some new Yb I lines on these spectrograms in the region longer than $9000 \AA$.

This analysis is thus based mainly on the wavelengths, intensities and Zeeman patterns published by Meggers and Corliss [1966] and on the infrared wavelengths and intensities reported by Humphreys and Paul [1959].

Meggers and Scribner [1937] reported that as early as 1930 three energy levels with separations of $703.5 \mathrm{~cm}^{-1}$ and $1718.4 \mathrm{~cm}^{-1}$ were detected from repeated differences among the wavenumbers. These were immediately interpreted by H. N. Russell as the intervals of the term $4 f^{14} 6 s 6 p{ }^{3} \mathrm{P}^{\circ}$. He also identified two singlet terms, $4 f^{14} 6 s 6 p^{1} \mathrm{P}^{\circ}$ and the ground term $4 f^{14} 6 s^{2}{ }^{2} \mathrm{~S}_{0}$, The raie ultime $3987.990 \AA$ was thus classified as this singlet transition ${ }^{1} \mathrm{~S}_{0}-{ }^{1} \mathrm{P}_{1}^{\circ}$. Eight terms and 22 classified lines were reported by Meggers and Scribner in the 1937 paper, all of which formed the beginning of the present work. At that time, the authors pointed out that Zeeman observations were needed to extend the analysis of the spectrum. The Zeeman observations are discussed in section 4.1. 
The lowest nine atomic energy levels of $\mathrm{Yb}$ I with their observed $g$-values are listed in the abstract by Meggers and Corliss [1960], which concludes with the statement that "nearly all of the strong lines of $\mathrm{YbI}$ are found to be combinations of these nine levels with some 70 higher energy levels. In that abstract, the ${ }^{3} \mathrm{D}$ and ${ }^{1} \mathrm{D}$ terms from the configuration $4 f^{14} 5 d 6$ s were added for the first time to the list of levels published in 1937.

Until 1964 the analysis of $\mathrm{Yb}$ I did not extend beyond the identification of levels belonging to two-electron configurations of the type $4 f^{14} n l n l^{\prime}$, which produce a spectrum resembling that of an alkaline earth. Meggers then encouraged N. Spector to perform some theoretical calculations to predict the positions of several configurations of the type $4 f^{13} n l n l^{\prime} n l^{\prime \prime}$, in which one of the $4 f$ electrons is excited. As a result of these calculations and a number of computer searches, Spector [1971] was able to identify for the first time levels belonging to the configuration $4 f^{13} 5 d 6 s^{2}$, thus shedding new light on the probable positions of such important configurations as $4 f^{13} 5 d 6 s 6 p, 4 f^{13} 5 d^{2} 6 s$, and $4 f^{13} 6 s^{2} 6 p$.

During the years of fruitful collaboration on the analysis of $\mathrm{Yb}$ II with the late G. Racah [1967], there was also some discussion of work on YbI. In 1964 Racah requested the new list of levels in order to complete the theoretical interpretation. A letter to Racah from Meggers in 1965 stated that the analysis of $\mathrm{Yb}$ I had been extended to include classifications for a large number of the 1800 observed lines.

After Racah's death in 1966, Z. B. Goldschmidt agreed to continue his theoretical work on $\mathrm{YbI}$ and began to construct the energy matrices for the high even configurations $f^{13}\left(d^{2}+d s+s^{2}\right) p$. In the same year, Meggers sent his first list of levels to her and noted that "this is the first complex spectrum in my experience that has more even than odd levels."

\section{Structure of the Spectrum}

Neutral ytterbium has two systems of energy levels: (1) those arising from electron configurations of the type $4 f^{14} n \ln l^{\prime}$, in which the $4 f$ electrons form a closed shell, and (2) those from configurations of the type $4 f^{13} n l n l^{\prime} n l^{\prime \prime}$, in which one of the $4 f$ electrons is excited. The configurations involving the closed $4 f$ shell produce a spectrum resembling that of an alkaline earth. In all the neutral lanthanides, the lowest configuration of the first type is $4 f^{\mathrm{N}} 6 s^{2}$ and the lowest of the second type is $4 f^{\mathrm{N}-1} 5 d 6 s^{2}$.

The ground state of neutral ytterbium is $4 f^{14} 6 s^{2}{ }^{1} \mathrm{~S}_{0}$. It combines strongly with the $4 f^{14} 6 s 6 p^{1,3} \mathrm{P}^{\circ}$ terms, as well as with the higher terms of the 6snp series. In the present observations this series has been observed to $n=13$. In absorption it has been observed by Camus and Tomkins [1969] to $n=48$. The first series number, $4 f^{14} 6 s^{2}{ }^{1} \mathrm{~S}_{0}-$ $4 f^{14} 6 s 6 p{ }^{1} \mathrm{P}_{0}^{\circ}$ at $3988 \AA$, is the strongest line of $\mathrm{Yb} \mathrm{I}$, and the second member $6 s^{2}{ }^{1} \mathrm{~S}_{0}-6 s 7 p{ }^{1} \mathrm{P}_{0}^{\circ}$ at $2464 \AA$, is the fifth strongest line according to Meggers, Corliss, and Scribner [1975].

The configuration $4 f^{14} 6 s 6 p$ in turn combines with the series 6sns which has been observed to $n=13$. The first member of the triplet series, $4 f^{14} 6 s 6 p{ }^{3} \mathrm{P}_{2}^{\circ}-4 f 6 s 7 s{ }^{3} \mathrm{~S}_{1}$ at $7699 \AA$, is the fourth strongest line of the spectrum. The $6 s 6 p$ configuration is also observed to combine with the 6snd series of terms to $n=9$.
S. Nir [1970] identified higher members of the 6sns, 6snp, and 6snd series by using calculations based on the Ritz-Rydberg formula and by analogy with known data on similar configurations in Eu I and Ba I.

As with the heavy elements of group II of the periodic table, intersystem transitions are strong in the two-electron spectrum of $\mathrm{Yb}$ I. The resonance intersystem transition $6 s^{2}{ }^{1} \mathrm{~S}_{0}$ - $6 s 6 p{ }^{3} \mathrm{P}_{1}^{\circ}$ at $5556 \AA$ is actually the third strongest line in the spectrum. It gives rise to the brilliant characteristic green color of an ytterbium arc or metal vapor lamp.

The lowest configuration of the rare-earth type, in which one of the $4 f$ electrons is excited, is $4 f^{13} 5 d 6 s^{2}$ starting at 23 $188 \mathrm{~cm}^{-1}$. There are 20 levels grouped into four terms in $J_{1} j$ coupling. The $\left(3^{1 / 2}, 1^{1 / 2}\right)^{\circ}$ and $\left(3^{1 / 2}, 2^{1 / 2}\right)^{\circ}$ terms in the lower half of the configuration are known, but the $\left(2^{1 / 2}\right.$, $\left.1^{1 / 2}\right)^{\circ}$ and $\left(2^{1 / 2}, 2^{1 / 2}\right)^{\circ}$ terms, which have been calculated to lie between 33000 and $41000 \mathrm{~cm}^{-1}$, have not been found. The transition from the level $\left(3^{1 / 2}, 2^{1 / 2}\right)_{1}^{\circ}$ at $28857 \mathrm{~cm}^{-1}$ to the ground state produces the second strongest line in the spectrum. The high intensity of this transition is qualitatively explained by the fact that the calculation in $L S$ coupling shows that the level at $28857 \mathrm{~cm}^{-1}$ is 17 percent $6 s 6{ }^{1} \mathrm{P}_{1}^{\circ}$. These calculations were made by S. Nir and Z. B. Goldschmidt (unpublished). N. Spector [1971], who originally collaborated with Meggers in identifying this configuration, has interpreted it as being $J_{1} l$-coupled, but the calculations of Nir and Goldschmidt indicate somewhat higher purity in $J_{1} j$ coupling.

The rare-earth type configuration $4 f^{13} 6 s^{2} 6 p$ starts at 32 $065 \mathrm{~cm}^{-1}$ and has been calculated in $J_{1} j$ coupling by Nir and Goldschmidt (unpublished) and, in part, in $J_{1} l$ coupling by Spector [1971]. Ten of the twelve levels of this configuration have been found. In $1967 \mathrm{Z}$. Goldschmidt noted in a letter to Meggers that "a prominent feature of all $4 f^{13} 6 s^{2} 6 p$ levels is the weakness of their transitions (with only a few exceptions) both to the low odd levels belonging to the configuration $4 f^{14} 6 s 6 p$ and to the high (as yet unidentified) odd levels. The forbidden transitions $4 f^{13} 6 s^{2} 6 p$ to $4 f^{14} 6 s 6 p$ exist as a result of configuration interaction of the types $4 f^{13} 6 s^{2} 6 p+4 f^{13} 5 d 6 s 6 p$ and/or $4 f^{14} 6 s 6 p+4 f^{13} 5 d 6 s^{2} . "$

The positions of energy levels in the configurations $4 f^{13} 6 s^{2} 6 p$ and $4 f^{13} 5 d 6 s^{2}$ are shown in figures 2 and 3 , respectively. Dashed bars denote levels that have not yet been found, and the positions shown are based on unpublished calculations by Nir and Goldschmidt.

Using a photoionization mass spectrometer, Parr and Elder [1968] measured the photoionization efficiency of ytterbium in the range 1350-2000 A and tentatively analyzed the observed line structure as arising from the configurations $4 f^{14} 5 d n p, 4 f^{14} 6 p n d, 4 f^{13} 6 s^{2} n d$, and $4 f^{13} 5 d 6 s n d$. Their identification of lines involving the configuration $4 f^{14} 5 d 6 p$ places the levels ${ }^{3} \mathrm{D}_{1}^{\circ},{ }^{3} \mathrm{P}_{1}^{\circ}$, and ${ }^{1} \mathrm{P}_{1}^{\circ}$ of this configuration above the ionization limit.

The relative positions of the configurations discussed above are displayed in figure 4 .

\section{Ionization Potential}

The most accurate value for the ionization potential for $\mathrm{Yb}$ I has been determined from absorption spectra by Camus and Tomkins [1969] using the series $6 s^{2}{ }^{1} \mathrm{~S}_{0} \rightarrow 6 s n p{ }^{1} \mathrm{P}_{1}^{\circ}$ for values of $n$ from 6 to 48 . They obtained the value 50441.0 


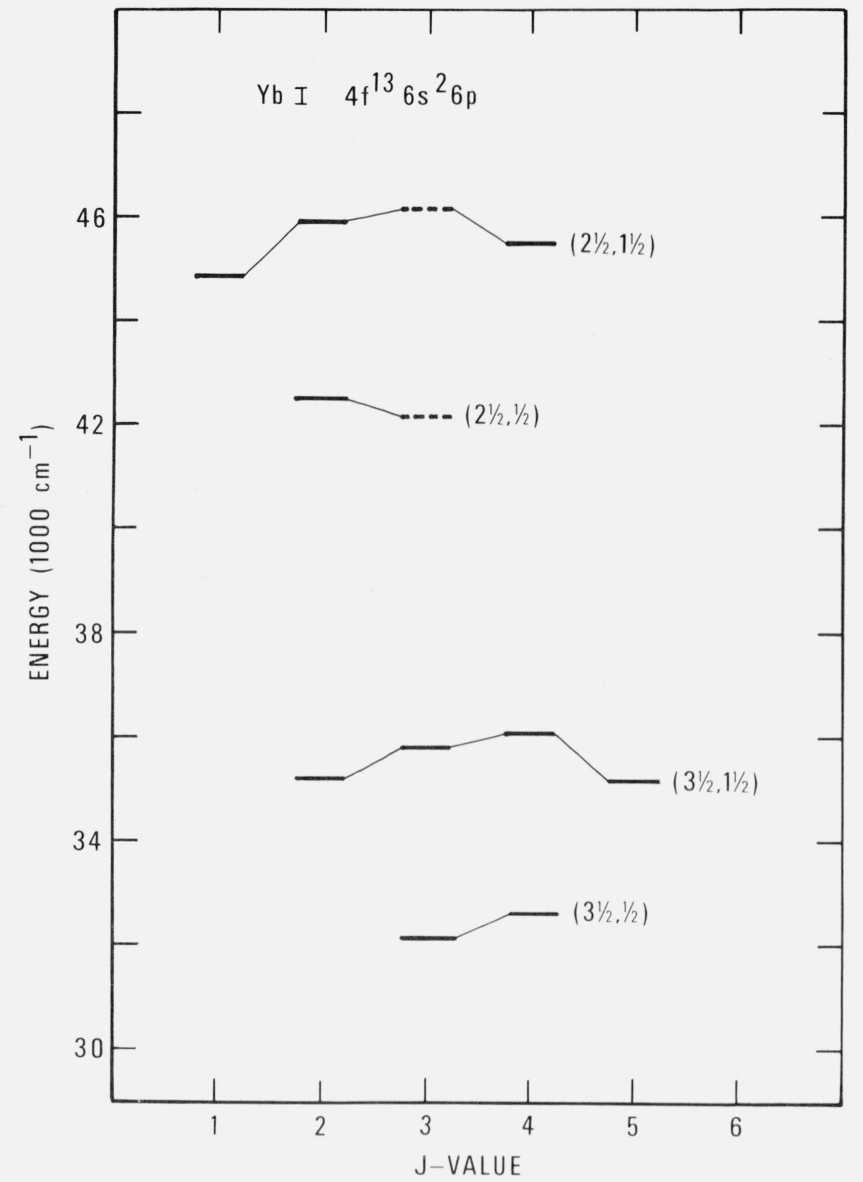

FigurE 2. Schematic representation of the energy levels of the configuration $4 \mathrm{f}^{13} 6 \mathrm{~s}^{2} 6 \mathrm{p}$.

The positions of known levels are represented by solid horizontal bars. Dashed bars indicate alculated positions of levels that have not yet been found. Levels are connected into terms in the $J_{j}$ coupling scheme.

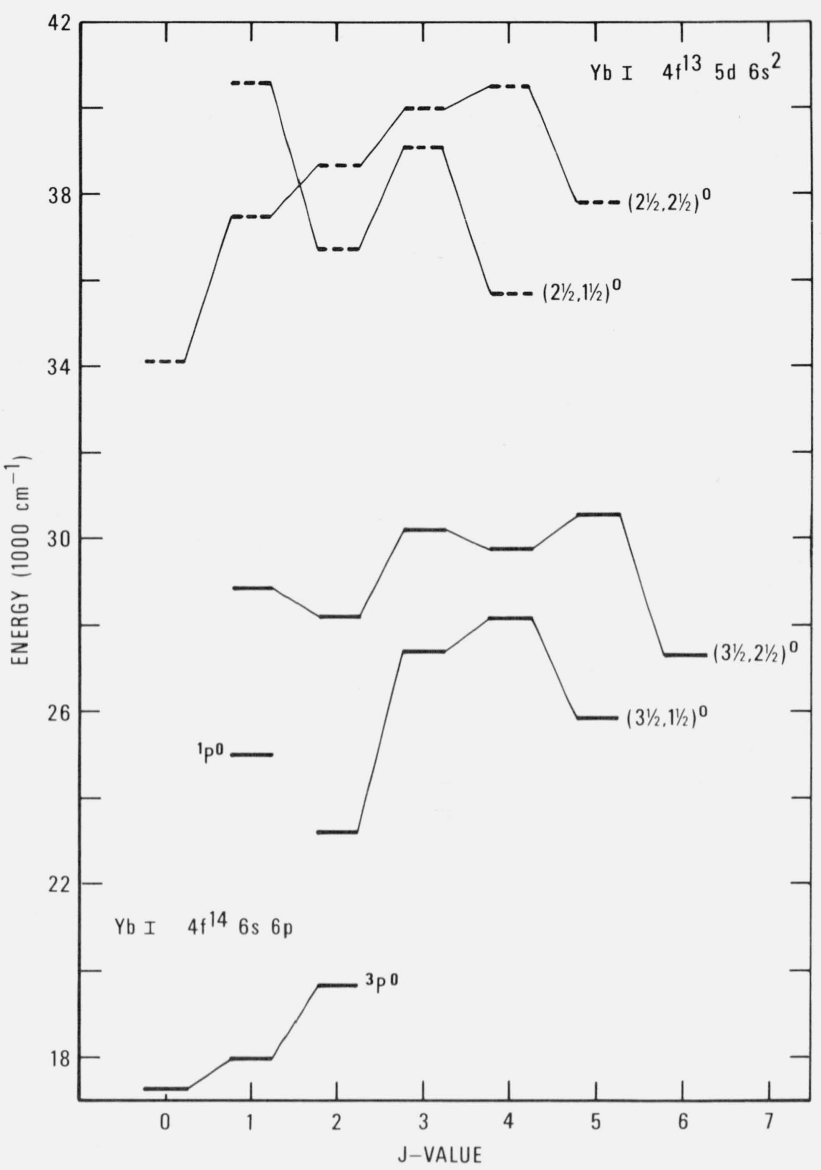

Figure 3. Energy level diagram for the configurations $4 \mathrm{f}^{13} 5 \mathrm{~d} 6 \mathrm{~s}^{2}$ and $4 f^{14} 6 s 6 p$.

Levels are connected into terms in the $\mathrm{J}_{1} j$ coupling scheme. Dashed bars indicate calculated positions of levels that have not yet been found.

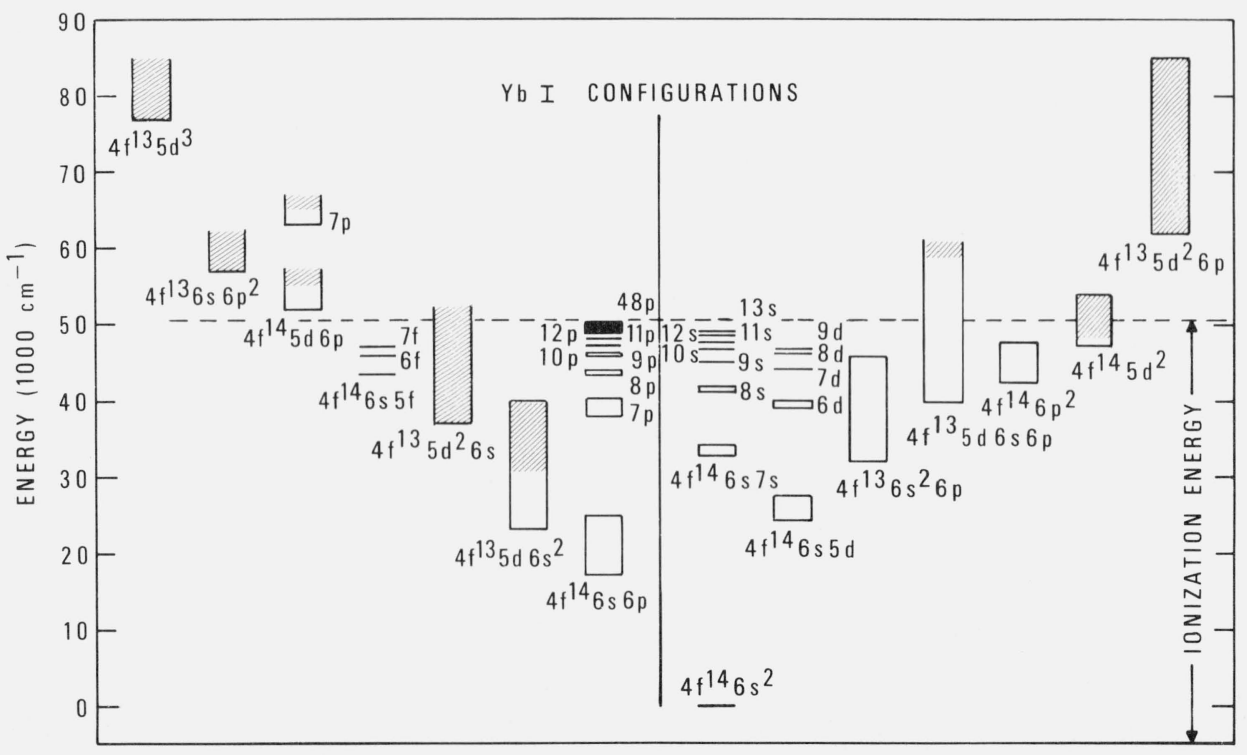

FigURE 4. Relative positions of known and predicted configurations in $Y b \mathrm{I}$. The cross-hatching indicates calculated positions of levels that have not yet been found. 
$\pm 0.2 \mathrm{~cm}^{-1}$ for the limit, which is the ground state $4 f^{14} 6 \mathrm{~s}^{2} \mathrm{~S}_{1 / 2}$ of $\mathrm{Yb}$ II. The conversion factor $8065.479 \pm 0.021 \mathrm{~cm}^{-1} / \mathrm{eV}$ has been used to calculate an ionization potential: 6.25394 $\pm 0.00003 \mathrm{eV}$. This value is in agreement with the value $6.25 \mathrm{eV}$ calculated by Reader and Sugar [1966] from the 6sns series.

\section{Tables}

\subsection{Zeeman Effect}

A major contribution to this analysis was provided by the Zeeman effect. The first set of observations of $\mathrm{Yb}$ Zeeman effect was made by Meggers at M.I.T. with the Bitter magnet in 1939. The high excitation provided by the $\mathrm{d}-\mathrm{c}$ arc in the large magnetic field produced Zeeman patterns for $\mathrm{Yb}$ II and Yb III but none for Yb I. The later development of electrodeless lamps, which produce lower excitation, paved the way for the first observation of Zeeman patterns for $\mathrm{Yb}$ I as described in detail by Meggers and Corliss [1966].

All of the observed Zeeman data are tabulated in table 1. Column 1 contains the wavelength as given in the list of observed lines (table 4 ). Column 2 gives the type of pattern. The standard Zeeman types, 1, 2, 3 for odd multiplicity and Type 7 which has a single undisplaced parallel $(p)$ component and two symmetrically displaced normal $(n)$ components, are excellently illustrated in a paper on hafnium spectra by Corliss and Meggers [1958]. Columns 3 and 4 list the measured splitting, in Lorentz units, of the observed $p$ and $n$ components, respectively. Except for resolved patterns these data refer to the strongest $p$ and $n$ components. Italics denote the splitting of the strong components in the case of a resolved pattern. Columns 5 and 6 give the observed $g$-values for the low and high levels, respectively, involved in the transition producing the line (see table 4). For example, the line at $5955.34 \AA$ is classified as $27445_{3}^{\circ}-$ $44232_{4}$. In table 1 the "lst $g$ ", 1.24 , is an observed $g$-value of the level $27445_{3}^{\circ}$, the "2nd $g$ ", 1.48, is that of the level $44232_{4}$.

In the case of unresolved patterns, it is often possible to derive a $g$-value for one level if the $g$-value for the other is already known. In the table, values that have been assumed in deriving the other are enclosed in parentheses.

Most of the final $g$-values were derived by Meggers, who completed the measurement and reduction of all Zeeman observations and interpreted practically all of the resolved patterns. A number of additional $g$-values from unresolved patterns have been derived by Charlotte E. Moore as described above.

The technique of optical double resonance has been used by Baumann and Wandel [1968] (BW) and by Budick and Snir [1967] (BS) to measure very accurate $g$-values for several levels of $\mathrm{Yb} \mathrm{I}$, as listed below. The values derived by Meggers (WFM) are in excellent agreement with these.

\begin{tabular}{|c|c|c|}
\hline Level & $g$-value & Reference \\
\hline $4 f^{14} 6 s 6 p{ }^{3} \mathrm{P}_{1}^{\circ}$ & $\begin{array}{l}1.49285(5) \\
1.49280(4) \\
1.48\end{array}$ & $\begin{array}{l}\text { BW } \\
\text { BS } \\
\text { WFM }\end{array}$ \\
\hline $4 f^{14} 6 s 6 p{ }^{1} \mathrm{P}_{1}^{\circ}$ & $\begin{array}{l}1.035(5) \\
1.03\end{array}$ & $\begin{array}{l}\text { BW } \\
\text { WFM }\end{array}$ \\
\hline $4 f^{13} 5 d 6 s^{2}\left(3 \frac{1}{2}, 1 \frac{1}{2}\right)_{1}^{\circ}$ & $\begin{array}{l}1.2635(9) \\
1.26\end{array}$ & $\begin{array}{l}\text { BS } \\
\text { WFM }\end{array}$ \\
\hline
\end{tabular}

\subsection{Energy Levels}

The even levels and terms of $\mathrm{Yb} \mathrm{I}$ are listed in table 2 and the odd levels and terms in table 3. Each table has six columns containing the following data: (1) configuration, (2) term designation, (3) $J$-value, (4) level value in $\mathrm{cm}^{-1}$, (5) observed $g$-value, and (6) number of observed combinations. Since levels with $J=0$ do not have a defined $g$-value, a Z in column 5 has been used for such a level to denote that the Zeeman effect has been observed for lines involving that level.

A number of levels in the original working list of Meggers have been rejected as spurious or unconfirmed. The present list includes, also, a number of important new levels added to Meggers' list in the present work. A few changes in the $J$ values assigned by Meggers have also been made.

The configuration and term assignments for many of the even levels have been generously provided by $Z$. Goldschmidt in advance of her publication of the theoretical interpretation of these configurations. For some of these levels, the calculated eigenvector purities are so low that meaningful names cannot be assigned. In such cases, only a configuration assignment has been made. The remaining unassigned odd levels undoubtedly belong for the most part to the configuration $4 f^{13} 5 d^{2} 6 s$.

The two even levels at $42437 \mathrm{~cm}^{-1}$ are peculiar in several ways. It was believed at first that this pair of levels was actually a single one, but that assumption resulted in a spread of observed-minus-calculated positions for the lines classified by this level that is much greater than for other levels. We concluded, therefore, that we are dealing with a pair of very close levels and have tentatively identified the upper member of the pair as $4 f^{14} 6 p^{2}{ }^{3} \mathrm{P}_{0}^{\circ}$. This interpretation is also supported by the isotope shifts of spectral lines involving these levels as measured by Miller and Ross [1976]. It should be noted that the Zeeman pattern of each of the observed lines involving these levels is anomalous in some respect.

The first three members of the $4 f^{14} 6 s n f^{3} \mathrm{~F}_{2}^{\circ}$ series have been tentatively identified. As in Yb II [1973] the quantum defects of these series members are close to unity, as expected. 
TABLE 1. Zeeman effect of $\mathrm{Yb} \mathrm{I}$

\begin{tabular}{|c|c|c|c|c|c|c|c|c|c|}
\hline \multirow{2}{*}{$\begin{array}{c}\text { Wavelength } \\
\text { (air) } \\
\AA\end{array}$} & \multirow{2}{*}{ Type } & \multicolumn{6}{|c|}{ Zeeman components (L.U.) } & \multirow{2}{*}{ lst $g$} & \multirow{2}{*}{ 2nd $g$} \\
\hline & & \multicolumn{3}{|c|}{$p$} & \multicolumn{3}{|c|}{$n$} & & \\
\hline 8325.18 & 3 & 0.81 & & & 1.04 & 1.45 & & 1.45 & 1.04 \\
\hline 8091.73 & 1 & 0.00 & 0.24 & & 0.76 & $1.00 ?$ & & 1.47 & 1.23 \\
\hline 8039.83 & 1 & 0.00 & 0.60 & & 0.00 & & & $(1.22)$ & 1.82 \\
\hline 7922.40 & 1 & 0.00 & 0.36 & & 0.34 & & & 1.44 & 1.08 \\
\hline 7895.08 & 2 & 0.00 & 0.13 & & 1.30 & & & 1.17 & 1.04 \\
\hline 7758.04 & 7 & 0.00 & & & 1.01 & & & 1.01 & 1.01 \\
\hline 7734.53 & 3 & 0.52 & & & 0.50 & 1.02 & & 0.50 & 1.02 \\
\hline 7699.49 & 1 & 0.00 & 0.50 & & 0.99 & & & 1.49 & 1.99 \\
\hline 7679.91 & 1 & 0.00 & 0.24 & & 0.62 & & & 1.10 & 1.34 \\
\hline 7527.45 & 1 & 0.00 & 0.17 & & 0.99 & & & 1.33 & 1.50 \\
\hline 7448.28 & 7 & 0.00 & & & 1.18 & & & $(1.16)$ & 1.14 \\
\hline 7350.04 & 7 & 0.00 & & & 0.49 & & & 0.49 & 0.00 \\
\hline 7327.87 & 1 & 0.00 & 0.38 & & & & & (1.14) & 1.52 \\
\hline 7313.05 & 2 & $0.00 \mathrm{~W}$ & & & 1.25 & & & (1.16) & 1.07 \\
\hline 7305.23 & 3 & 0.64 & & & & & & $(0.50)$ & 1.14 \\
\hline 7244.41 & 3 & 0.67 & & & 1.17 & 1.50 & & 1.17 & 1.50 \\
\hline 7187.07 & 1 & 0.00 & & & 0.94 & & & & \\
\hline 6799.60 & 3 & 0.51 & & & 1.50 & 2.01 & & 1.50 & 2.01 \\
\hline 6782.17 & 3 & 0.52 & & & 0.50 & 1.02 & & 1.02 & 0.50 \\
\hline 6768.70 & 2 & 0.00 & 0.13 & & 1.30 & & & 1.04 & 1.17 \\
\hline 6765.24 & 7 & 0.00 & & & 1.04 & & & 1.09 & (1.08) \\
\hline 6749.40 & 2 & 0.00 & 0.79 & & 2.62 & & & 1.04 & 1.83 \\
\hline 6715.79 & 7 & 0.00 & & & 1.18 & & & 1.18 & 1.18 \\
\hline 6692.42 & 1 & 0.00 & 0.30 & & 0.20 & & & 1.10 & 1.40 \\
\hline 6678.17 & 3 & 0.45 & & & 1.01 & 1.25 & & 1.01 & 1.25 \\
\hline 6667.82 & 7 & 0.00 & & & 1.03 & & & 1.02 & 1.03 \\
\hline 6643.55 & 7 & 0.00 & & & 1.01 & & & 1.01 & 1.01 \\
\hline 6626.73 & 2 & 0.00 & 0.21 & & 1.66 & & & 1.24 & 1.03 \\
\hline 6607.07 & 1 & 0.00 & 0.21 & & 0.69 & & & 1.09 & 1.29 \\
\hline 6555.15 & 7 & $0.00 \mathrm{w}$ & & & 1.26 & & & 1.16 & (1.14) \\
\hline 6550.14 & 3 & 0.08 & & & $1.30 \mathrm{w}$ & & & & \\
\hline 6489.06 & 2 & 0.00 & & & 2.02 & & & 0.00 & 2.02 \\
\hline 6421.53 & 2 & $0.00 \mathrm{w}$ & & & 1.25 & & & 1.10 & (1.14) \\
\hline 6417.91 & 2 & 0.00 & & & 1.15 & & & (1.01) & 1.06 \\
\hline 6404.62 & 1 & 0.00 & 0.44 & & 0.57 & & & 1.01 & 1.45 \\
\hline 6400.35 & 7 & 0.00 & & & 1.04 & & & (1.01) & 1.02 \\
\hline 6393.73 & 2 & $0.00 \mathrm{~W}$ & & & 1.48 & & & (1.09) & 1.17 \\
\hline 6372.71 & 1 & 0.00 & 0.11 & & 0.76 & & & 1.09 & 1.20 \\
\hline 6344.97 & 3 & 0.64 & & & 0.68 & 1.00 & & 1.00 & 0.68 \\
\hline 6335.72 & 1 & 0.00 & 0.13 & & 0.96 & & & 1.21 & 1.34 \\
\hline 6286.25 & 2 & $0.00 \mathrm{w}$ & & & 1.44 & & & 1.25 & $(1.34)$ \\
\hline 6247.99 & 3 & 0.18 & & & & & & 1.09 & (1.14) \\
\hline 6194.84 & 2 & 0.00 & 0.20 & & 1.63 & & & 1.02 & 1.22 \\
\hline 6181.78 & 7 & 0.00 & & & 1.26 & & & $(1.23)$ & 1.24 \\
\hline 6118.28 & 7 & 0.00 & & & 1.02 & & & (1.01) & 1.00 \\
\hline 6111.27 & 2 & 0.00 & & & 1.52 & & & 1.52 & 0.00 \\
\hline 6065.72 & 2 & 0.00 & & & 1.35 & & & 1.25 & $(1.30)$ \\
\hline 6054.57 & 7 & 0.00 & & & 1.13 & & & (1.18) & 1.17 \\
\hline 6048.44 & 1 & 0.00 & 0.24 & & 0.43 & & & 1.15 & 1.39 \\
\hline 6035.72 & 7 & 0.00 & & & 1.17 & & & $(1.18)$ & 1.16 \\
\hline 6031.80 & 3 & 0.74 & & & 1.02 & 1.39 & & 1.02 & 1.39 \\
\hline 6014.95 & 1 & 0.00 & 0.25 & & 0.36 & & & 1.11 & 1.36 \\
\hline 6004.52 & 3 & 0.66 & & & 1.15 & 1.48 & & 1.48 & 1.15 \\
\hline 6003.62 & 1 & $0.00 \mathrm{~W}$ & & & $1.04+$ & & & & \\
\hline 5989.33 & 3 & 0.72 & & & 1.48 & 1.84 & & 1.48 & 1.84 \\
\hline 5959.33 & 2 & $0.00 w$ & & & 1.26 & & & (1.01) & 1.09 \\
\hline 5958.70 & 1 & 0.00 & 0.12 & & 1.09 & & & 1.45 & 1.33 \\
\hline 5955.34 & 2 & 0.00 & 0.24 & 0.47 & 1.73 & 1.96 & 2.20 & 1.24 & 1.48 \\
\hline
\end{tabular}


TABLE 1. Zeeman effect of $\mathrm{Yb} \mathrm{I}$ - Continued

\begin{tabular}{|c|c|c|c|c|c|c|}
\hline \multirow{2}{*}{$\begin{array}{c}\text { Wavelength } \\
\text { (air) } \\
\AA\end{array}$} & \multirow{2}{*}{ Type } & \multicolumn{3}{|c|}{ Zeeman components (L.U.) } & \multirow{2}{*}{ lst $g$} & \multirow{2}{*}{ 2nd $g$} \\
\hline & & $p$ & \multicolumn{2}{|c|}{$n$} & & \\
\hline 5950.66 & 3 & 0.20 & $1.13 w$ & & 1.15 & 1.10 \\
\hline 5936.49 & 1 & $0.00 \mathrm{~W}$ & 0.42 & & 1.01 & $(0.86)$ \\
\hline 5925.46 & 2 & 0.00 & 1.03 & & 1.03 & 0.00 \\
\hline 5854.510 & 3 & 0.74 & 0.89 & 1.04 & 1.04 & 0.89 \\
\hline 5831.82 & 3 & 0.56 & 1.04 & 1.32 & 1.04 & 1.32 \\
\hline 5810.67 & 1 & 0.00 & 0.62 & & 1.48 & 1.05 \\
\hline 5803.44 & 2 & $0.00 \mathrm{~W}$ & 1.31 & & (1.14) & 1.18 \\
\hline 5755.89 & $3 ?$ & 0.32 & 0.72 & 1.36 & & \\
\hline 5749.91 & 3 & 0.16 & 1.19 & & 1.18 & 1.21 \\
\hline 5745.80 & 1 & 0.00 & 0.83 & & 1.01 & 1.19 \\
\hline 5728.853 & 7 & 0.00 & 1.11 & & & \\
\hline 5724.58 & 1 & 0.00 & 0.98 & & (1.03) & 1.00 \\
\hline 5719.99 & 3 & 0.00 & 0.99 & & (1.01) & 0.97 \\
\hline 5701.922 & 3 & 0.20 & $1.15 \mathrm{~W}$ & & 1.17 & 1.13 \\
\hline 5699.95 & 1 & 0.00 & 0.68 & & 1.20 & 1.07 \\
\hline 5689.917 & 7 & 0.00 & 1.16 & & & \\
\hline 5683.09 & 7 & 0.00 & 1.14 & & 1.14 & 1.14 \\
\hline 5597.189 & 7 & 0.00 & 1.05 & & (1.09) & 1.08 \\
\hline 5586.362 & 3 & 0.14 & 1.21 & & 1.17 & 1.24 \\
\hline 5578.232 & 7 & 0.00 & 1.23 & & & \\
\hline 5568.11 & 2 & $0.00 \mathrm{w}$ & 1.30 & & $(1.04)$ & 1.08 \\
\hline 5562.093 & 3 & 0.29 & 1.02 & 1.17 & 1.17 & 1.02 \\
\hline 5556.466 & 2 & 0.00 & 1.48 & & 0.00 & 1.48 \\
\hline 5539.053 & 1 & $0.00 \mathrm{~W}$ & 1.01 & & & \\
\hline 5524.544 & 7 & 0.00 & 1.16 & & (1.16) & 1.16 \\
\hline 5505.49 & 2 & 0.00 & 2.00 & & 0.50 & 1.25 \\
\hline 5498.75 & 7 & 0.00 & 1.01 & & & \\
\hline 5493.088 & 2 & 0.32 & 2.00 & & 1.04 & 1.36 \\
\hline 5481.925 & 2 & 0.52 & 1.55 & & 0.49 & 1.02 \\
\hline 5474.037 & 1 & $0.00 \mathrm{w}$ & 1.06 & & 1.18 & (1.21) \\
\hline 5454.007 & 1 & $0.00 \mathrm{w}$ & 0.97 & & $(1.22)$ & 1.15 \\
\hline 5403.079 & 1 & $0.00 \mathrm{~W}$ & 0.88 & & $(1.16)$ & 1.06 \\
\hline 5393.757 & 1 & $0.00 \mathrm{~W}$ & 1.01 & & $(1.34)$ & 1.49 \\
\hline 5390.845 & 3 & 0.33 & $1.27 \mathrm{~W}$ & & 1.33 & 1.22 \\
\hline 5390.622 & 1 & $0.00 \mathrm{~W}$ & 0.77 & & $(1.16)$ & 1.03 \\
\hline 5380.54 & 2 & $0.00 \mathrm{w}$ & 1.56 & & (1.08) & 1.20 \\
\hline 5380.24 & 7 & 0.00 & 0.98 & & (1.08) & 1.10 \\
\hline 5363.66 & 2 & $0.00 \mathrm{~W}$ & 1.66 & & $(1.45)$ & 1.51 \\
\hline 5351.29 & 3 & 0.96 & 0.68 & 1.15 & 1.15 & 0.68 \\
\hline 5323.10 & 1 & 0.40 & 0.74 & & 1.14 & 1.54 \\
\hline 5299.852 & 3 & 0.81 & 0.86 & 1.06 & 1.06 & 0.86 \\
\hline 5288.51 & 7 & 0.00 & 1.21 & & $(1.14)$ & 1.16 \\
\hline 5287.45 & 1 & 0.32 & 0.83 & & 1.15 & 1.47 \\
\hline 5277.04 & 2 & $0.00 \mathrm{w}$ & 0.85 & & $(0.50)$ & 0.68 \\
\hline 5275.592 & 3 & 0.30 & 1.20 & 1.30 & 1.20 & 1.30 \\
\hline 5244.11 & 2 & $0.00 \mathrm{~W}$ & 1.27 & & (1.16) & 1.20 \\
\hline 5228.172 & 7 & $0.00 w$ & 1.16 & & $(1.34)$ & $1.30 ?$ \\
\hline 5227.271 & 1 & $0.00 \mathrm{~W}$ & 0.87 & & 1.04 & $(1.08)$ \\
\hline 5214.95 & 3 & 0.99 & 0.49 & 1.48 & 0.49 & 1.48 \\
\hline 5211.604 & 3 & 0.75 & 1.10 & 1.35 & 1.35 & 1.10 \\
\hline 5196.085 & 3 & 0.60 & 1.15 & 1.25 & 1.15 & 1.25 \\
\hline 5193.850 & $1 ?$ & 0.28 & 1.17 & 1.74 & $(1.45)$ & $1.73 ?$ \\
\hline 5182.755 & 7 & 0.00 & $1.18 ?$ & & $(1.03)$ & 1.10 \\
\hline 5139.53 & 1 & $0.00 w$ & 0.92 & & $(1.14)$ & 1.10 \\
\hline 5126.80 & 3 & 0.42 & & & $(1.16)$ & 1.37 \\
\hline 5113.34 & 3 & 0.65 & $1.09 \mathrm{~W}$ & & 1.03 & 1.16 \\
\hline 5104.85 & 1 & 0.00 & & & & \\
\hline 5080.981 & 2 & $0.00 w$ & 1.34 & & (1.14) & 1.06 \\
\hline
\end{tabular}


TABLE 1. Zeeman effect of $\mathrm{Yb} \mathrm{I}$ - Continued

\begin{tabular}{|c|c|c|c|c|c|c|c|}
\hline \multirow{2}{*}{$\begin{array}{c}\text { Wavelength } \\
\text { (air) } \\
\AA\end{array}$} & \multirow{2}{*}{ Type } & \multicolumn{4}{|c|}{ Zeeman components (L.U.) } & \multirow{2}{*}{ lst $g$} & \multirow{2}{*}{ 2nd $g$} \\
\hline & & \multicolumn{2}{|c|}{$p$} & \multicolumn{2}{|c|}{$n$} & & \\
\hline 5076.744 & 2 & 0.00 & 0.32 & 1.66 & & 1.02 & 1.34 \\
\hline 5074.34 & 1 & 0.00 & & 1.04 & & $(1.16)$ & 1.12 \\
\hline 5069.144 & 7 & 0.00 & & 1.17 & & $(1.16)$ & 1.18 \\
\hline 5067.800 & 2 & $0.00 \mathrm{~W}$ & & 1.50 & & (1.14) & 1.21 \\
\hline 5058.613 & 2 & 0.00 & 0.88 & 2.27 & & 0.51 & 1.39 \\
\hline 5043.708 & 7 & 0.00 & & 0.99 & & $(1.01)$ & 1.03 \\
\hline 5027.67 & 2 & 0.00 & 0.35 & 2.04 & & 1.34 & 0.99 \\
\hline 5019.691 & 1 & 0.00 & 0.10 & 0.60 & & 1.00 & 1.10 \\
\hline 4974.16 & 2 & 0.00 & 1.01 & 2.52 & & 1.51 & 0.50 \\
\hline 4966.902 & 3 & 0.69 & & 1.16 & 1.51 & 1.51 & 1.16 \\
\hline 4956.512 & 3 & 0.66 & & 1.52 & 1.85 & 1.52 & 1.85 \\
\hline 4935.500 & 1 & 0.00 & 0.17 & 0.99 & & 1.50 & 1.33 \\
\hline 4931.953 & 2 & 0.00 & 0.28 & 1.58 & & 1.02 & 1.30 \\
\hline 4918.118 & 7 & 0.00 & & 1.22 & & 1.22 & 1.22 \\
\hline 4912.365 & 3 & 0.96 & & 1.04 & 1.52 & 1.52 & 1.04 \\
\hline 4899.78 & 3 & 0.36 & & 0.97 & 1.15 & 1.15 & 0.97 \\
\hline 4893.465 & 7 & 0.00 & & $1.12 ?$ & & (1.16) & $1.20 ?$ \\
\hline 4891.992 & 7 & 0.00 & & 1.41 & & & \\
\hline 4853.826 & 1 & $0.00 \mathrm{w}$ & & 0.96 & & (1.16) & 1.20 \\
\hline 4837.46 & 2 & 0.00 & 0.48 & 1.47 & & 0.51 & 0.99 \\
\hline 4831.30 & 3 & 0.78 & & 0.50 & 1.28 & 0.50 & 1.28 \\
\hline 4816.43 & 1 & 0.00 & 0.28 & 0.24 & & & \\
\hline 4812.918 & 3 & 0.28 & & 1.00 & 1.14 & 1.00 & 1.14 \\
\hline 4781.867 & 1 & 0.00 & 0.15 & 0.80 & & 1.40 & 1.25 \\
\hline 4780.32 & 1 & 0.00 & 0.17 & 0.98 & & 1.33 & 1.50 \\
\hline 4778.982 & 3 & 0.35 & & 1.02 & 1.11 & 1.11 & 1.02 \\
\hline 4762.587 & 2 & W & & $1.84 \mathrm{~V}$ & & & \\
\hline 4758.320 & 7 & 0.00 & & 0.94 & & & \\
\hline 4751.789 & 1 & $0.00 \mathrm{~W}$ & & 0.60 & & (1.14) & 1.32 \\
\hline 4743.356 & 3 & 0.19 & & $1.17 \mathrm{~V}$ & & 1.15 & 1.20 \\
\hline 4720.79 & 2 & $0.00 \mathrm{~W}$ & 0.54 & 1.85 & & (1.04) & 1.18 \\
\hline 4718.56 & $7 ?$ & $0.00 \mathrm{w}$ & & 1.02 & & & \\
\hline 4687.593 & $1 ?$ & 0.17 & & 1.00 & & 1.17 & 1.34 \\
\hline 4684.268 & 7 & 0.00 & & 1.24 & & $(1.22)$ & 1.22 \\
\hline 4666.735 & 7 & 0.00 & & 1.13 & & (1.16) & 1.19 \\
\hline 4656.971 & 2 & 0.00 & 0.21 & 0.93 & & 0.51 & 0.72 \\
\hline 4651.67 & 3 & 0.40 & & $1.19 \mathrm{w}$ & & 1.16 & 1.22 \\
\hline 4650.05 & 7 & 0.00 & & 1.13 & & (1.16) & 1.15 \\
\hline 4644.54 & 1 & 0.00 & & 1.27 & & $(1.45)$ & 1.39 \\
\hline 4624.41 & 2 & $0.00 \mathrm{w}$ & & 1.26 & & (1.16) & 1.06 \\
\hline 4615.947 & 1 & $0.00 \mathrm{~W}$ & & 0.53 & & $(1.22)$ & 1.04 \\
\hline 4610.172 & 3 & 0.72 & & 0.52 & 1.24 & 0.52 & 1.24 \\
\hline 4590.834 & 3 & 0.21 & & $1.08 \mathrm{~V}$ & & 1.06 & 1.10 \\
\hline 4589.211 & 2 & 0.00 & 0.25 & 1.84 & & 1.34 & 1.09 \\
\hline 4585.916 & 7 & 0.00 & & 0.98 & & & \\
\hline 4582.355 & 3 & 0.99 & & 0.50 & 1.50 & 1.50 & 0.50 \\
\hline 4580.724 & $7 ?$ & 0.00 & & 1.13 & & & \\
\hline 4576.209 & 1 & 0.00 & 0.33 & 0.80 & & 1.46 & 1.13 \\
\hline 4568.853 & 3 & 0.57 & & 0.50 & 1.07 & 0.50 & 1.07 \\
\hline 4567.368 & 2 & 0.00 & 0.32 & 2.16 & & 1.52 & 1.84 \\
\hline 4563.95 & 1 & 0.00 & 0.50 & 1.02 & & 1.52 & 2.02 \\
\hline 4533.506 & 3 & 0.90 & & 1.04 & 1.22 & 1.04 & 1.22 \\
\hline 4531.333 & 1 & $0.00 \mathrm{w}$ & & 1.06 & & & \\
\hline 4529.87 & 1 & 0.00 & 0.46 & 0.57 & & 1.49 & 1.03 \\
\hline 4513.408 & 3 & 0.30 & & 1.30 & 1.45 & 1.45 & 1.30 \\
\hline 4503.636 & 3 & 0.45 & & 1.05 & 1.14 & 1.05 & 1.14 \\
\hline 4488.282 & 1 & 0.00 & 0.24 & 0.73 & & 1.45 & 1.21 \\
\hline 4482.422 & 3 & 0.16 & & 1.08 & 1.16 & 1.16 & 1.08 \\
\hline
\end{tabular}


TABLE 1. Zeeman effect of $\mathrm{Yb}$ I-Continued

\begin{tabular}{|c|c|c|c|c|c|c|c|}
\hline \multirow{2}{*}{$\begin{array}{c}\text { Wavelength } \\
\text { (air) } \\
\AA\end{array}$} & \multirow{2}{*}{ Type } & \multicolumn{4}{|c|}{ Zeeman components (L.U.) } & \multirow{2}{*}{ lst $g$} & \multirow{2}{*}{2 nd $g$} \\
\hline & & \multicolumn{2}{|c|}{$p$} & \multicolumn{2}{|c|}{$n$} & & \\
\hline 4472.470 & 7 & 0.00 & & 1.06 & & $(1.03)$ & 1.04 \\
\hline 4439.19 & 2 & 0.00 & & 0.51 & & 0.00 & 0.51 \\
\hline 4430.208 & $3 ?$ & $0.66 ?$ & & 0.40 & $1.06 ?$ & & \\
\hline 4411.095 & $3 ?$ & 0.54 & & $1.26 \mathrm{~W}$ & & & \\
\hline 4402.605 & 7 & 0.00 & & 1.03 & & & \\
\hline 4398.96 & $1 ?$ & 0.23 & & 1.29 & & $(1.50)$ & $1.73 ?$ \\
\hline 4396.254 & 7 & 0.00 & & 1.06 & & & \\
\hline 4393.688 & 7 & 0.00 & & 1.05 & & (1.03) & 1.04 \\
\hline 4376.456 & 3 & 0.45 & & 0.50 & 0.95 & 0.50 & 0.95 \\
\hline 4359.528 & 3 & 0.88 & & 0.48 & 1.36 & 0.48 & 1.36 \\
\hline 4352.948 & 3 & 0.25 & & $1.28 \mathrm{~W}$ & & 1.32 & 1.24 \\
\hline 4329.718 & 7 & $0.00 \mathrm{~h}$ & & $1.08 \mathrm{~h}$ & & & \\
\hline 4326.404 & 1 & 0.00 & 0.12 & 0.98 & & 1.11 & 1.23 \\
\hline 4309.823 & 7 & 0.00 & & 0.49 & & & \\
\hline 4305.966 & 3 & 0.27 & & 1.24 & 1.33 & 1.33 & 1.24 \\
\hline 4300.984 & 7 & 0.00 & & 1.02 & & $(1.04)$ & 1.04 \\
\hline 4284.170 & 7 & 0.00 & & 1.01 & & & \\
\hline 4277.738 & 3 & 0.80 & & 0.50 & 1.30 & 0.50 & 1.30 \\
\hline 4251.521 & 3 & 0.30 & & 1.34 & 1.49 & 1.49 & 1.34 \\
\hline 4233.445 & 1 & $0.00 \mathrm{w}$ & & 1.04 & & $(1.01)$ & 1.02 ? \\
\hline 4231.972 & 3 & 0.51 & & 1.51 & 2.02 & 1.51 & 2.02 \\
\hline 4218.693 & 7 & 0.00 & & 1.15 & & 1.15 & 1.15 \\
\hline 4211.82 & 2 & 0.00 & & 1.30 & & (1.16) & 1.21 \\
\hline 4210.299 & 7 & 0.00 & & 1.21 & & & \\
\hline 4174.56 & 2 & 0.00 & & 1.50 & & 1.50 & 0.00 \\
\hline 4149.066 & 7 & 0.00 & & 1.51 & & 1.51 & 1.51 \\
\hline 4109.574 & 2 & 0.00 & & 2.01 & & 0.00 & 2.01 \\
\hline 4089.68 & $3 ?$ & 0.34 & & 1.47 & 1.82 & & \\
\hline 4059.470 & 7 & 0.00 & & 1.20 & & & \\
\hline 4052.283 & 1 & 0.00 & 0.17 & 0.99 & & 1.49 & 1.32 \\
\hline 4052.072 & 1 & 0.00 & 0.41 & 0.19 & & 1.44 & 1.02 \\
\hline 4007.356 & 3 & 0.14 & & 1.37 & 1.44 & 1.44 & 1.37 \\
\hline 3993.753 & 3 & 0.30 & & $1.19 \mathrm{~W}$ & & & \\
\hline 3990.885 & 3 & 0.30 & & 1.34 & 1.49 & 1.49 & 1.34 \\
\hline 3987.99 & 7 & 0.00 & & 1.02 & & 0.00 & 1.02 \\
\hline 3975.283 & $2 ?$ & 0.32 & & 1.74 & & 0.00 & 1.74 ? \\
\hline 3961.98 & 1 & 0.00 & 0.14 & 1.20 & & 1.47 & 1.33 \\
\hline 3911.272 & 3 & 0.30 & & $1.13 \mathrm{w}$ & & 1.16 & 1.10 \\
\hline 3900.85 & 3 & 0.37 & & 1.31 & 1.50 & 1.50 & 1.31 \\
\hline 3872.852 & 7 & 0.00 & & 1.47 & & 1.47 & 1.47 \\
\hline 3839.907 & 1 & 0.00 & 0.14 & 1.04 & & 1.47 & 1.33 \\
\hline 3838.287 & 2 & 0.00 & 0.48 & 0.97 & & & \\
\hline 3791.741 & 1 & 0.00 & 0.38 & 0.71 & & 1.47 & 1.09 \\
\hline 3774.323 & 2 & 0.00 & 0.20 & 2.22 & & & \\
\hline 3770.095 & 7 & 0.00 & & 1.46 & & 0.00 & 1.46 \\
\hline 3736.237 & 3 & 0.71 & & 1.12 & 1.48 & 1.48 & 1.12 \\
\hline 3734.694 & 1 & 0.00 & 0.14 & 1.21 & & 1.49 & 1.35 \\
\hline 3734.435 & 1 & 0.00 & 0.16 & 1.00 & & & \\
\hline 3706.023 & $3 ?$ & 0.66 & & 1.33 & & & \\
\hline 3700.580 & 2 & 0.00 & 0.07 & 1.51 & & 1.07 & 1.14 \\
\hline 3684.997 & 3 & 0.50 & & 1.48 & 1.98 & 1.48 & 1.98 \\
\hline 3666.62 & 7 & 0.00 & & 0.85 & & & \\
\hline 3655.729 & 1 & 0.00 & 0.18 & 1.11 & & 1.47 & 1.29 \\
\hline 3629.233 & 7 & 0.00 & & 0.66 & & 0.00 & 0.66 \\
\hline 3614.994 & 7 & 0.00 & & 1.13 & & (1.09) & 1.10 \\
\hline 3578.561 & 1 & $0.00 \mathrm{~W}$ & & 0.96 & & (1.50) & 1.32 \\
\hline 3559.032 & 7 & 0.00 & & 1.48 & & 1.48 & 0.00 \\
\hline 3517.001 & 3 & 0.21 & & $1.41 \mathrm{w}$ & & 1.46 & 1.36 \\
\hline
\end{tabular}


TABLE 1. Zeeman effect of $\mathrm{Yb} \mathrm{I}-$ Continued

\begin{tabular}{|c|c|c|c|c|c|c|c|}
\hline \multirow{2}{*}{$\begin{array}{c}\text { Wavelength } \\
\text { (air) } \\
\AA\end{array}$} & \multirow{2}{*}{ Type } & \multicolumn{4}{|c|}{ Zeeman Components (L.U.) } & \multirow{2}{*}{ lst $g$} & \multirow{2}{*}{ 2nd $g$} \\
\hline & & 1 & & & & & \\
\hline 3513.573 & 3 & 0.95 & \multirow{17}{*}{0.33} & 0.49 & \multirow[t]{17}{*}{1.44} & 1.44 & 0.49 \\
\hline 3510.764 & 1 & 0.00 & & 0.80 & & 1.46 & 1.13 \\
\hline 3464.37 & 7 & 0.00 & & 1.26 & & 0.00 & 1.26 \\
\hline 3460.269 & 7 & 0.00 & & 1.07 & & & \\
\hline 3459.663 & 7 & 0.00 & & 1.04 & & & \\
\hline 3452.398 & 3 & 0.21 & & 1.11 & & 1.09 & 1.14 \\
\hline 3443.587 & 7 & 0.00 & & 1.18 & & & \\
\hline 3431.140 & 7 & 0.00 & & 1.09 & & & \\
\hline 3431.107 & 7 & 0.00 & & 1.12 & & & \\
\hline 3426.044 & 7 & 0.00 & & 1.00 & & & \\
\hline 3418.390 & 7 & 0.00 & & 1.04 & & (1.14) & 1.12 \\
\hline 3412.453 & 7 & 0.00 & & 1.13 & & (1.14) & 1.13 \\
\hline 3387.505 & 1 & $0.00 \mathrm{w}$ & & 0.96 & & & \\
\hline 3319.412 & 7 & 0.00 & & 1.16 & & & \\
\hline 3316.496 & $i$ & $0.00 \mathrm{w}$ & & 1.28 & & $(1.48)$ & 1.38 \\
\hline 3299.828 & 7 & 0.00 & & 0.73 & & 0.00 & 0.73 \\
\hline 2671.958 & 7 & 0.00 & & 1.01 & & 0.00 & 1.01 \\
\hline
\end{tabular}


TABLE 2. Even levels of $\mathrm{YbI}$

\begin{tabular}{|c|c|c|c|c|c|}
\hline Configuration & $\begin{array}{l}\text { Desig- } \\
\text { nation }\end{array}$ & $J$ & $\begin{array}{l}\text { Level } \\
\mathrm{cm}^{-1}\end{array}$ & $\begin{array}{l}\text { Obs. } \\
g\end{array}$ & $\begin{array}{c}\text { No. } \\
\text { Comb. }\end{array}$ \\
\hline $4 f^{14}\left({ }^{1} \mathrm{~S}\right) 6 s^{2}$ & ${ }^{1} \mathrm{~S}$ & 0 & 0.000 & $\mathrm{Z}$ & 11 \\
\hline $4 f^{14}\left({ }^{1} \mathrm{~S}\right) 5 d 6 s$ & ${ }^{3} \mathrm{D}$ & $\begin{array}{l}1 \\
2 \\
3\end{array}$ & $\begin{array}{l}24489.102 \\
24751.948 \\
25270.902\end{array}$ & $\begin{array}{l}0.50 \\
1.16 \\
1.34\end{array}$ & $\begin{array}{l}41 \\
47 \\
31\end{array}$ \\
\hline $4 f^{14}\left({ }^{1} \mathrm{~S}\right) 5 d 6 s$ & ${ }^{1} \mathrm{D}$ & 2 & 27677.665 & 1.01 & 43 \\
\hline $4 f^{13}\left({ }^{2} \mathrm{~F}_{3 \frac{1}{2}}^{\circ}\right) 6 s^{2} 6 p$ & $\left(3 \frac{1}{2}, \frac{1}{2}\right)$ & $\begin{array}{l}3 \\
4\end{array}$ & $\begin{array}{l}32065.282 \\
32273.597\end{array}$ & 1.23 & $\begin{array}{l}18 \\
15\end{array}$ \\
\hline $4 f^{14}\left({ }^{1} \mathrm{~S}\right) 6 s 7 s$ & ${ }^{3} \mathrm{~S}$ & 1 & 32694.692 & 2.01 & 24 \\
\hline $4 f^{14}\left({ }^{1} \mathrm{~S}\right) 6 s 7 s$ & ${ }^{1} \mathrm{~S}$ & 0 & 34350.65 & Z & 10 \\
\hline $4 f^{13}\left({ }^{2} \mathrm{~F}_{3 \frac{1}{2}}^{\circ}\right) 6 s^{2} 6 p$ & $\left(3 \frac{1}{2}, 1 \frac{1}{2}\right)$ & $\begin{array}{l}5 \\
2 \\
3 \\
4\end{array}$ & $\begin{array}{l}35178.78 \\
35196.98 \\
35807.52 \\
36060.98\end{array}$ & $\begin{array}{l}1.05 \\
1.08\end{array}$ & $\begin{array}{r}8 \\
12 \\
13 \\
13\end{array}$ \\
\hline $4 f^{14}\left({ }^{1} \mathrm{~S}\right) 6 s 6 d$ & ${ }^{3} \mathrm{D}$ & $\begin{array}{l}1 \\
2 \\
3\end{array}$ & $\begin{array}{l}39808.72 \\
39838.04 \\
39966.09\end{array}$ & $\begin{array}{l}0.50 \\
1.16 \\
1.33\end{array}$ & $\begin{array}{l}5 \\
6 \\
3\end{array}$ \\
\hline $4 f^{13}\left({ }^{2} \mathrm{~F}_{3 \frac{1}{2}}^{\circ}\right) 5 d\left({ }^{2} \mathrm{D}\right) 6 s 6 p\left({ }^{3} \mathrm{P}^{\circ}\right)\left({ }^{4} \mathrm{~F}_{1 \frac{1}{2}}^{\circ}\right)$ & $\left(3 \frac{1}{2}, 1 \frac{1}{2}\right)$ & $\begin{array}{l}2 \\
5 \\
4 \\
3\end{array}$ & $\begin{array}{l}39880.26 \\
42935.78 \\
44984.75 \\
45462.54\end{array}$ & $\begin{array}{l}1.83 \\
0.89 \\
1.08 \\
1.20\end{array}$ & $\begin{array}{l}6 \\
6 \\
8 \\
7\end{array}$ \\
\hline $4 f^{14}\left({ }^{1} \mathrm{~S}\right) 6 s 6 d$ & ${ }^{1} \mathrm{D}$ & 2 & 40061.51 & 1.03 & 5 \\
\hline $4 f^{14}\left({ }^{1} \mathrm{~S}\right) 6 s 8 s$ & ${ }^{3} \mathrm{~S}$ & 1 & 41615.04 & 2.02 & 5 \\
\hline
\end{tabular}


TABLE 2. Even levels of $\mathrm{YbI}-$ Continued

\begin{tabular}{|c|c|c|c|c|c|}
\hline Configuration & $\begin{array}{l}\text { Desig- } \\
\text { nation }\end{array}$ & $J$ & $\begin{array}{l}\text { Level } \\
\mathrm{cm}^{-1}\end{array}$ & $\begin{array}{c}\text { Obs. } \\
g\end{array}$ & $\begin{array}{c}\text { No. } \\
\text { Comb. }\end{array}$ \\
\hline $4 f^{13}\left({ }^{2} \mathrm{~F}_{3 \frac{1}{2}}^{\circ}\right) 5 d\left({ }^{2} \mathrm{D}\right) 6 s 6 p\left({ }^{3} \mathrm{P}^{\circ}\right)\left({ }^{4} \mathrm{~F}_{2 \frac{1}{2}}^{\circ}\right)$ & $\left(3 \frac{1}{2}, 2 \frac{1}{2}\right)$ & $\begin{array}{l}3 \\
1 \\
6 \\
5 \\
4\end{array}$ & $\begin{array}{l}41827.30 \\
42436.70 \\
43814.11 \\
45410.91 \\
45775.68\end{array}$ & $\begin{array}{l}1.52 \\
1.73 \\
1.08 \\
1.17 \\
1.14\end{array}$ & $\begin{array}{l}7 \\
5 \\
9 \\
6 \\
8\end{array}$ \\
\hline $4 f^{14}\left({ }^{1} \mathrm{~S}\right) 6 s 8 s$ & ${ }^{1} \mathrm{~S}$ & 0 & 41939.90 & Z & 3 \\
\hline $4 f^{14}\left({ }^{1} \mathrm{~S}\right) 6 p^{2}$ & ${ }^{3} \mathrm{P}$ & $\begin{array}{l}0 \\
1 \\
2\end{array}$ & $\begin{array}{l}42436.91 \\
43805.42 \\
44760.37\end{array}$ & $\begin{array}{c}\mathrm{Z} \\
1.47 \\
1.34\end{array}$ & $\begin{array}{l}2 \\
7 \\
8\end{array}$ \\
\hline $4 f^{13}\left({ }^{2} \mathrm{~F}_{2 \frac{1}{2}}^{\circ}\right) 6 s^{2} 6 p$ & $\left(2 \frac{1}{2}, \frac{1}{2}\right)$ & $\begin{array}{l}3 \\
2\end{array}$ & 42531.87 & 1.01 & 7 \\
\hline $4 f^{13} 5 d 6 s 6 p$ & & 2 & 43224.78 & 1.34 & 7 \\
\hline $4 f^{13} 5 d 6 s 6 p$ & & 4 & 44232.66 & 1.48 & 4 \\
\hline $4 f^{14}\left({ }^{1} \mathrm{~S}\right) 6 s 7 d$ & ${ }^{3} \mathrm{D}$ & $\begin{array}{l}1 \\
2 \\
3\end{array}$ & $\begin{array}{l}44311.38 \\
44313.05 \\
44380.82\end{array}$ & 1.32 & $\begin{array}{l}5 \\
6 \\
5\end{array}$ \\
\hline $4 f^{14}\left({ }^{1} \mathrm{~S}\right) 6 s 7 d$ & ${ }^{1} \mathrm{D}$ & 2 & 44357.60 & 1.10 & 5 \\
\hline $4 f^{13} 5 d 6 s 6 p$ & & 3 & 44713.12 & 1.39 & 6 \\
\hline $4 f^{13}\left({ }^{2} \mathrm{~F}_{2 \frac{1}{2}}^{\circ}\right) 6 s^{2} 6 p$ & $\left(2 \frac{1}{2}, 1 \frac{1}{2}\right)$ & $\begin{array}{l}1 \\
4 \\
2 \\
3\end{array}$ & $\begin{array}{l}44834.61 \\
45497.62 \\
45913.86\end{array}$ & 0.66 & $\begin{array}{l}6 \\
5 \\
7\end{array}$ \\
\hline $4 f^{14}\left({ }^{1} \mathrm{~S}\right) 6 s 9 s$ & ${ }^{3} \mathrm{~S}$ & 1 & 45121.37 & 1.98 & 5 \\
\hline
\end{tabular}


TABLE 2. Even levels of $\mathrm{YhI}-$ Continued

\begin{tabular}{|c|c|c|c|c|c|}
\hline Configuration & $\begin{array}{l}\text { Desig- } \\
\text { nation }\end{array}$ & $J$ & $\begin{array}{l}\text { Level } \\
\mathrm{cm}^{-1}\end{array}$ & $\begin{array}{c}\text { Obs. } \\
g\end{array}$ & $\begin{array}{c}\text { No. } \\
\text { Comb. }\end{array}$ \\
\hline $4 f^{13}\left({ }^{2} \mathrm{~F}_{3 \frac{1}{2}}^{\circ}\right) 5 d\left({ }^{2} \mathrm{D}\right) 6 s 6 p\left({ }^{3} \mathrm{P}^{\circ}\right)\left({ }^{2} \mathrm{D}_{2 \frac{1}{2}}^{\circ}\right)$ & $\left(3 \frac{1}{2}, 2 \frac{1}{2}\right)$ & $\begin{array}{l}2 \\
6 \\
4\end{array}$ & $\begin{array}{l}45338.53 \\
47036.62 \\
48787.71\end{array}$ & $\begin{array}{l}1.30 \\
1.18 \\
1.21\end{array}$ & $\begin{array}{l}8 \\
6 \\
4\end{array}$ \\
\hline $4 f^{13}\left({ }^{2} \mathrm{~F}_{3 \frac{1}{2}}^{\circ}\right) 5 d\left({ }^{\circ} \mathrm{D}\right) 6 s 6 p\left({ }^{3} \mathrm{P}^{\circ}\right)\left({ }^{4} \mathrm{~F}_{3 \frac{1}{2}}^{\circ}\right)$ & $\left(3 \frac{1}{2}, 3 \frac{1}{2}\right)$ & $\begin{array}{l}1 \\
0 \\
4 \\
5 \\
6\end{array}$ & $\begin{array}{l}45595.14 \\
46081.54 \\
47772.94 \\
48057.77 \\
48688.18\end{array}$ & $\begin{array}{c}1.19 \\
\mathrm{Z} \\
1.22 \\
1.13\end{array}$ & $\begin{array}{l}7 \\
3 \\
5 \\
5 \\
6\end{array}$ \\
\hline $4 f^{13} 5 d 6 s 6 p$ & & 3 & 46395.60 & 1.35 & 7 \\
\hline $4 f^{13} 5 d 6 s 6 p$ & & 2 & 46431.49 & & 5 \\
\hline $4 f^{14}\left({ }^{1} \mathrm{~S}\right) 6 s 8 d$ & ${ }^{3} \mathrm{D}$ & $\begin{array}{l}1 \\
2 \\
3\end{array}$ & $\begin{array}{l}46444.96 \\
46467.70 \\
46480.65\end{array}$ & $\begin{array}{l}0.49 \\
1.12 \\
1.35\end{array}$ & $\begin{array}{l}5 \\
6 \\
2\end{array}$ \\
\hline $4 f^{13} 5 d 6 s 6 p$ & & 6 & 46554.81 & 1.25 & 7 \\
\hline $4 f^{14}\left({ }^{1} \mathrm{~S}\right) 6 s 10 s$ & ${ }^{3} \mathrm{~S}$ & 1 & 46877.10 & & 3 \\
\hline $4 f^{13}\left({ }^{2} \mathrm{~F}_{3 \frac{1}{2}}^{\circ}\right) 5 d\left({ }^{2} \mathrm{D}\right) \operatorname{ss} 6 p\left({ }^{3} \mathrm{P}^{\circ}\right)\left({ }^{4} \mathrm{D}_{\frac{1}{2}}^{\circ}\right)$ & $\left(3 \frac{1}{2}, \frac{1}{2}\right)$ & 4 & 47047.69 & 0.86 & 8 \\
\hline $4 f^{13}\left({ }^{2} \mathrm{~F}_{3 \frac{1}{2}}^{\circ}\right) 5 d\left({ }^{2} \mathrm{D}\right) 6 s 6 p\left({ }^{3} \mathrm{P}^{\circ}\right)\left({ }^{4} \mathrm{D}_{1 \frac{1}{2}}^{\circ}\right)$ & $\left(3 \frac{1}{2}, 1 \frac{1}{2}\right)$ & $\begin{array}{l}5 \\
3 \\
4\end{array}$ & $\begin{array}{l}47088.16 \\
47646.62 \\
47673.71\end{array}$ & $\begin{array}{l}1.16 \\
1.32\end{array}$ & $\begin{array}{l}8 \\
6 \\
6\end{array}$ \\
\hline $4 f^{14}\left({ }^{1} \mathrm{~S}\right) 5 d^{2}$ & & 2 & 47341.82 & & 5 \\
\hline $4 f^{14}\left({ }^{1} \mathrm{~S}\right) 6 s 9 d$ & ${ }^{1} \mathrm{D}$ & 2 & $\begin{array}{r}47420.96 \\
47584.34\end{array}$ & 0.73 & 7 \\
\hline
\end{tabular}


TABLE 2. Even levels of $\mathrm{Yb} \mathrm{I}-$ Continued

\begin{tabular}{|c|c|c|c|c|c|}
\hline Configuration & $\begin{array}{l}\text { Desig- } \\
\text { nation }\end{array}$ & $J$ & $\begin{array}{l}\text { Level } \\
\mathrm{cm}^{-1}\end{array}$ & $\begin{array}{c}\text { Obs. } \\
g\end{array}$ & $\begin{array}{c}\text { No. } \\
\text { Comb. }\end{array}$ \\
\hline $4 f^{14}\left({ }^{1} \mathrm{~S}\right) 5 d^{2}$ & ${ }^{3} \mathrm{~F}$ & $\begin{array}{l}2 \\
3 \\
4\end{array}$ & $\begin{array}{l}47634.41 \\
47860.28\end{array}$ & 1.02 & $\begin{array}{l}4 \\
7\end{array}$ \\
\hline & & 3 & 47673.45 & & 4 \\
\hline $4 f^{13} 5 d 6 s 6 p$ & & 5 & 47636.11 & 1.09 & 9 \\
\hline $4 f^{13} 5 d 6 s 6 p$ & & 1 & 47645.40 & & 7 \\
\hline $4 f^{14}\left({ }^{1} \mathrm{~S}\right) 6 p^{2}$ & ${ }^{1} \mathrm{D}$ & 2 & 47821.78 & 1.04 & 8 \\
\hline $4 f^{14}\left({ }^{1} \mathrm{~S}\right) 6 s 11 s$ & ${ }^{3} \mathrm{~S}$ & 1 & 47885.81 & & 3 \\
\hline \multirow[t]{4}{*}{$4 f^{13}\left({ }^{2} \mathrm{~F}_{3 \frac{1}{2}}^{\circ}\right) 5 d\left({ }^{2} \mathrm{D}\right) 6 s 6 p\left({ }^{3} \mathrm{P}^{\circ}\right)\left({ }^{2} \mathrm{D}_{1 \frac{1}{2}}^{\circ}\right)$} & $\left(3 \frac{1}{2}, 1 \frac{1}{2}\right)$ & $\begin{array}{l}5 \\
2 \\
4\end{array}$ & $\begin{array}{l}47911.48 \\
48135.60 \\
49103.64\end{array}$ & $\begin{array}{l}1.21 \\
1.37 \\
1.04\end{array}$ & $\begin{array}{l}8 \\
6 \\
7\end{array}$ \\
\hline & & 1 & 48309.41 & & 6 \\
\hline & & $1 ?$ & 48357.54 & & 5 \\
\hline & & 1 & 48360.43 & & 6 \\
\hline $4 f^{14}\left({ }^{1} \mathrm{~S}\right) 6 s 12 s$ & ${ }^{3} \mathrm{~S}$ & 1 & 48519.71 & & 2 \\
\hline $4 f^{13}\left({ }^{2} \mathrm{~F}_{3 \frac{1}{2}}^{\circ}\right) 5 d\left({ }^{2} \mathrm{D}\right) 6 s 6 p\left(\left({ }^{3} \mathrm{P}^{\circ}\right)\left({ }^{4} \mathrm{D}_{2 \frac{1}{2}}^{\circ}\right)\right.$ & $\left(3 \frac{1}{2}, 2 \frac{1}{2}\right)$ & $\begin{array}{l}3 \\
5\end{array}$ & $\begin{array}{l}48647.78 \\
49079.34\end{array}$ & & $\begin{array}{l}7 \\
9\end{array}$ \\
\hline $4 f^{13}\left({ }^{2} \mathrm{~F}_{3 \frac{1}{2}}^{\circ}\right) 5 d\left({ }^{2} \mathrm{D}\right) 6 s 6 P\left({ }^{3} \mathrm{P}^{\circ}\right)\left({ }^{4} \mathrm{D}_{3 \frac{1}{2}}^{\circ}\right)$ & $\left(3 \frac{1}{2}, 3 \frac{1}{2}\right)$ & $\begin{array}{l}6 \\
5\end{array}$ & $\begin{array}{l}48806.57 \\
49638.73\end{array}$ & 1.22 & $\begin{array}{l}8 \\
6\end{array}$ \\
\hline $4 f^{13}\left({ }^{2} \mathrm{~F}_{3 \frac{1}{2}}^{\circ}\right) 5 d\left({ }^{2} \mathrm{D}\right) 6 s 6 p\left({ }^{3} \mathrm{P}^{\circ}\right)\left({ }^{4} \mathrm{~F}_{4 \frac{1}{2}}^{\circ}\right)$ & $\left(3 \frac{1}{2}, 4 \frac{1}{2}\right)$ & $\begin{array}{l}1 ? \\
2\end{array}$ & $\begin{array}{l}48838.33 \\
48883.11\end{array}$ & & $\begin{array}{l}6 \\
6\end{array}$ \\
\hline $4 f^{14}\left({ }^{1} \mathrm{~S}\right) 6 s 13 s$ & ${ }^{3} \mathrm{~S}$ & 1 & 48943.42 & & 3 \\
\hline
\end{tabular}


TABle 2. Even levels of $\mathrm{YbI}-$ Continued

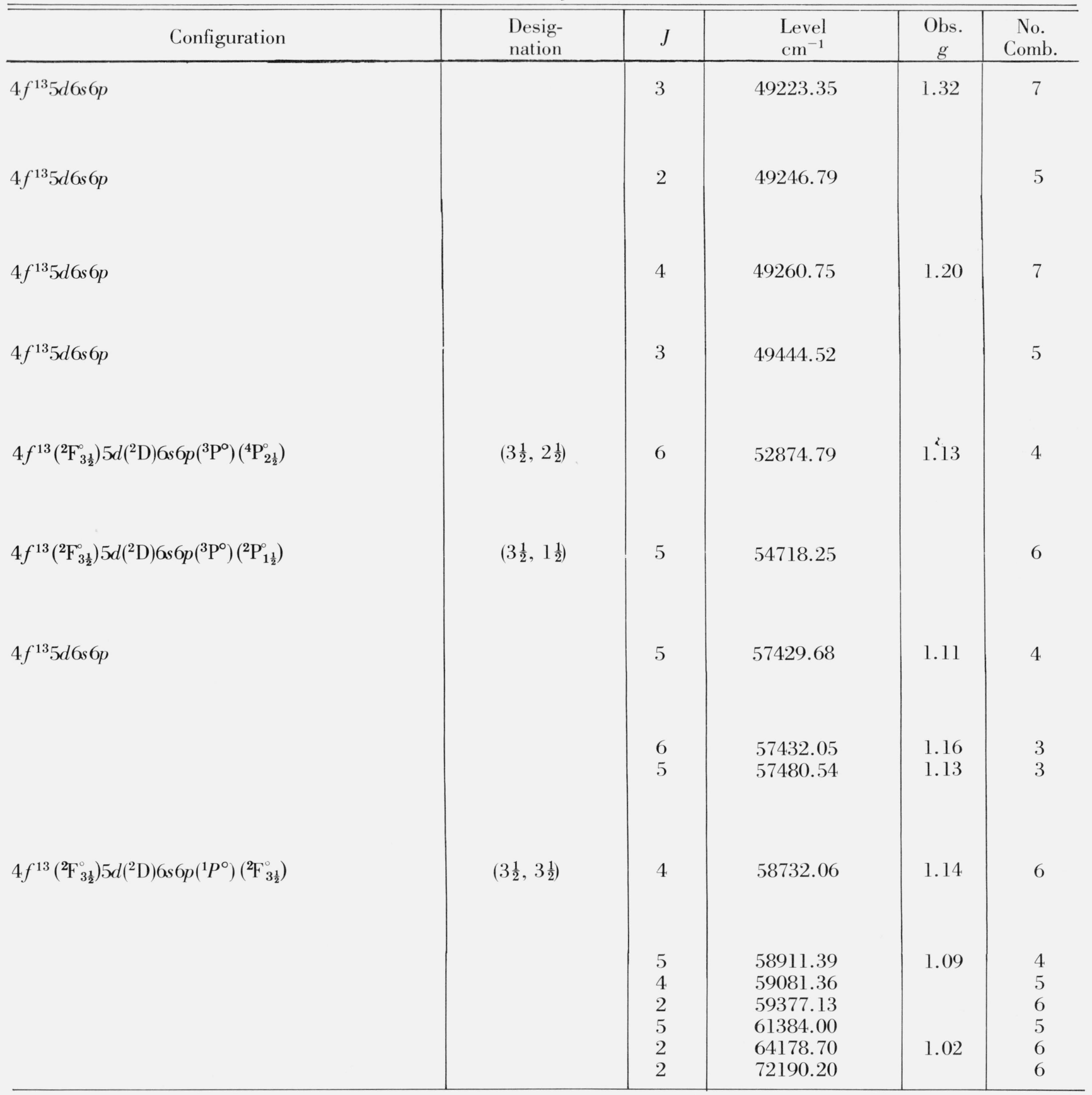


TABLE 3. Odd levels of $\mathrm{YbI}$

\begin{tabular}{|c|c|c|c|c|c|}
\hline Configuration & $\begin{array}{l}\text { Desig- } \\
\text { nation }\end{array}$ & $J$ & Level & $\begin{array}{l}\text { Obs. } \\
g\end{array}$ & $\begin{array}{l}\text { No. } \\
\text { Comb. }\end{array}$ \\
\hline $4 f^{14}\left({ }^{1} \mathrm{~S}\right) 6 s 6 p$ & ${ }^{3} \mathrm{P}^{\circ}$ & $\begin{array}{l}0 \\
1 \\
2\end{array}$ & $\begin{array}{l}17288.439 \\
17992.007 \\
19710.388\end{array}$ & $\begin{array}{c}\mathrm{Z} \\
1.48 \\
1.50\end{array}$ & $\begin{array}{l}18 \\
46 \\
53\end{array}$ \\
\hline $4 f^{13}\left({ }^{2} \mathrm{~F}_{3 \frac{1}{2}}^{\circ}\right) 5 d 6 s^{2}$ & $\left(3 \frac{1}{2}, 1 \frac{1}{2}\right)^{\circ}$ & $\begin{array}{l}2 \\
5 \\
3 \\
4\end{array}$ & $\begin{array}{l}23188.518 \\
25859.682 \\
27445.638 \\
28184.512\end{array}$ & $\begin{array}{l}1.45 \\
1.04 \\
1.22 \\
1.14\end{array}$ & $\begin{array}{l}41 \\
32 \\
38 \\
36\end{array}$ \\
\hline $4 f^{14}\left({ }^{1} \mathrm{~S}\right) 6 s 6 p$ & ${ }^{1} \mathrm{P}^{\circ}$ & 1 & 25068.227 & 1.03 & 35 \\
\hline $4 f^{13}\left({ }^{2} \mathrm{~F}_{3 \frac{1}{2}}^{\circ}\right) 5 d 6 s^{2}$ & $\left(3 \frac{1}{2}, 2 \frac{1}{2}\right)^{\circ}$ & $\begin{array}{l}6 \\
2 \\
1 \\
4 \\
3 \\
5\end{array}$ & $\begin{array}{l}27314.919 \\
28195.960 \\
28857.014 \\
29774.958 \\
30207.380 \\
30524.714\end{array}$ & $\begin{array}{l}1.16 \\
1.02 \\
1.26 \\
1.09 \\
1.08 \\
1.18\end{array}$ & $\begin{array}{l}19 \\
28 \\
30 \\
34 \\
31 \\
29\end{array}$ \\
\hline $4 f^{13}\left({ }^{2} \mathrm{~F}^{\circ}\right) 5 d^{2} 6 s ?$ & & 1 & 37414.59 & 1.02 & 7 \\
\hline $4 f^{14}\left({ }^{1} \mathrm{~S}\right) 6 s 7 p$ & ${ }^{3} \mathrm{P}^{\circ}$ & $\begin{array}{l}0 \\
1 \\
2\end{array}$ & $\begin{array}{l}38090.71 \\
38174.17 \\
38551.93\end{array}$ & $\begin{array}{l}1.14 \\
1.50\end{array}$ & $\begin{array}{l}3 \\
7 \\
9\end{array}$ \\
\hline $4 f^{13}\left({ }^{2} \mathrm{~F}^{\circ}\right) 5 d^{2} 6 s ?$ & & 1 & 38422.36 & 1.07 & 7 \\
\hline $4 f^{14}\left({ }^{1} \mathrm{~S}\right) 6 s 7 p$ & ${ }^{1} \mathrm{P}^{\circ}$ & 1 & 40563.97 & 1.01 & 6 \\
\hline & & $\begin{array}{l}2 \\
2 \\
3 \\
3\end{array}$ & $\begin{array}{l}42647.72 \\
42725.76 \\
43254.78 \\
43297.51\end{array}$ & $\begin{array}{l}1.25 \\
1.02 \\
1.06 \\
1.03\end{array}$ & $\begin{array}{l}4 \\
6 \\
3 \\
3\end{array}$ \\
\hline $4 f^{14}\left({ }^{1} \mathrm{~S}\right) 6 s 5 f$ & ${ }^{3} \mathbf{F}^{\circ}$ & $\begin{array}{l}2 \\
3 \\
4\end{array}$ & 43433.85 & 0.68 & 4 \\
\hline & & 1 & 43532.77 & 1.54 & 3 \\
\hline
\end{tabular}


TABLE 3. Odd levels of $\mathrm{YbI}-$ Continued

\begin{tabular}{|c|c|c|c|c|c|}
\hline Configuration & $\begin{array}{l}\text { Desig- } \\
\text { nation }\end{array}$ & $J$ & Level & $\begin{array}{c}\text { Obs. } \\
g\end{array}$ & $\begin{array}{c}\text { No. } \\
\text { Comb. }\end{array}$ \\
\hline \multirow[t]{2}{*}{$4 f^{14}\left({ }^{1} \mathrm{~S}\right) 6 s 8 p$} & ${ }^{3} \mathrm{P}^{\circ}$ & $\begin{array}{l}0 \\
1 \\
2\end{array}$ & $\begin{array}{l}43614.27 \\
43659.38 \\
43805.69\end{array}$ & $\begin{array}{l}1.48 \\
1.49\end{array}$ & $\begin{array}{l}2 \\
6 \\
6\end{array}$ \\
\hline & & 3 & 43815.67 & 1.21 & 5 \\
\hline \multirow[t]{6}{*}{$4 f^{14}\left({ }^{1} \mathrm{~S}\right) 6 s 8 p$} & ${ }^{1} \mathrm{P}^{\circ}$ & 1 & 44017.60 & 1.00 & 7 \\
\hline & & 2 & 44251.88 & 1.38 & 5 \\
\hline & & $4 ?$ & 44392.70 & 1.30 ? & 4 \\
\hline & & 3 & 44453.47 & 1.10 & 6 \\
\hline & & 2 & 45155.33 & 0.98 & 6 \\
\hline & & 1 & 45181.69 & 1.28 & 5 \\
\hline $4 f^{14}\left({ }^{1} \mathrm{~S}\right) 6 s 6 f$ & ${ }^{3} \mathrm{~F}^{\circ}$ & $\begin{array}{l}2 \\
3 \\
4\end{array}$ & 45956.27 & 0.72 & 6 \\
\hline \multirow[t]{2}{*}{$4 f^{14}\left({ }^{1} \mathrm{~S}\right) 6 s 9 p$} & ${ }^{3} \mathrm{P}^{\circ}$ & $\begin{array}{l}0 \\
1 \\
2\end{array}$ & $\begin{array}{l}46082.17 \\
46078.91 \\
46184.15\end{array}$ & $\begin{array}{l}1.34 \\
1.50\end{array}$ & $\begin{array}{l}2 \\
6 \\
5\end{array}$ \\
\hline & & $\begin{array}{l}1 \\
3\end{array}$ & $\begin{array}{l}46174.21 \\
46251.06\end{array}$ & $\begin{array}{l}1.22 \\
1.15\end{array}$ & $\begin{array}{l}4 \\
7\end{array}$ \\
\hline \multirow[t]{3}{*}{$4 f^{14}\left({ }^{1} \mathrm{~S}\right) 6 s 9 p$} & ${ }^{1} \mathrm{P}^{\circ}$ & 1 & 46370.30 & 1.07 & 5 \\
\hline & & 2 & 47055.05 & 1.08 & 5 \\
\hline & & $\begin{array}{l}3 ? \\
1\end{array}$ & $\begin{array}{l}47325.19 \\
47332.26\end{array}$ & 0.95 & $\begin{array}{l}5 \\
3\end{array}$ \\
\hline $4 f^{14}\left({ }^{1} \mathrm{~S}\right) 6 s 7 f$ & ${ }^{3} \mathrm{~F}^{\circ}$ & $\begin{array}{l}2 \\
3 \\
4\end{array}$ & 47326.65 & & 4 \\
\hline $4 f^{14}\left({ }^{1} \mathrm{~S}\right) 6 s 10 p$ & ${ }^{3} \mathrm{P}^{\circ}$ & $\begin{array}{l}0 \\
1 \\
2\end{array}$ & $\begin{array}{l}47409.82 \\
47420.93 \\
47471.10\end{array}$ & 1.36 & $\begin{array}{l}1 \\
2 \\
5\end{array}$ \\
\hline \multirow[t]{2}{*}{$4 f^{14}\left({ }^{1} \mathrm{~S}\right) 6 s 10 p$} & ${ }^{1} \mathrm{P}^{\circ}$ & 1 & 47498.83 & 1.03 & 4 \\
\hline & & $\begin{array}{l}3 ? \\
1 \\
4 \\
3\end{array}$ & $\begin{array}{l}47843.13 \\
47859.31 \\
47939.10 \\
48154.71 \\
\end{array}$ & 1.30 & $\begin{array}{l}6 \\
4 \\
6 \\
3 \\
\end{array}$ \\
\hline
\end{tabular}


TABLE 3. Odd levels of $\mathrm{YbI}-$ Continued

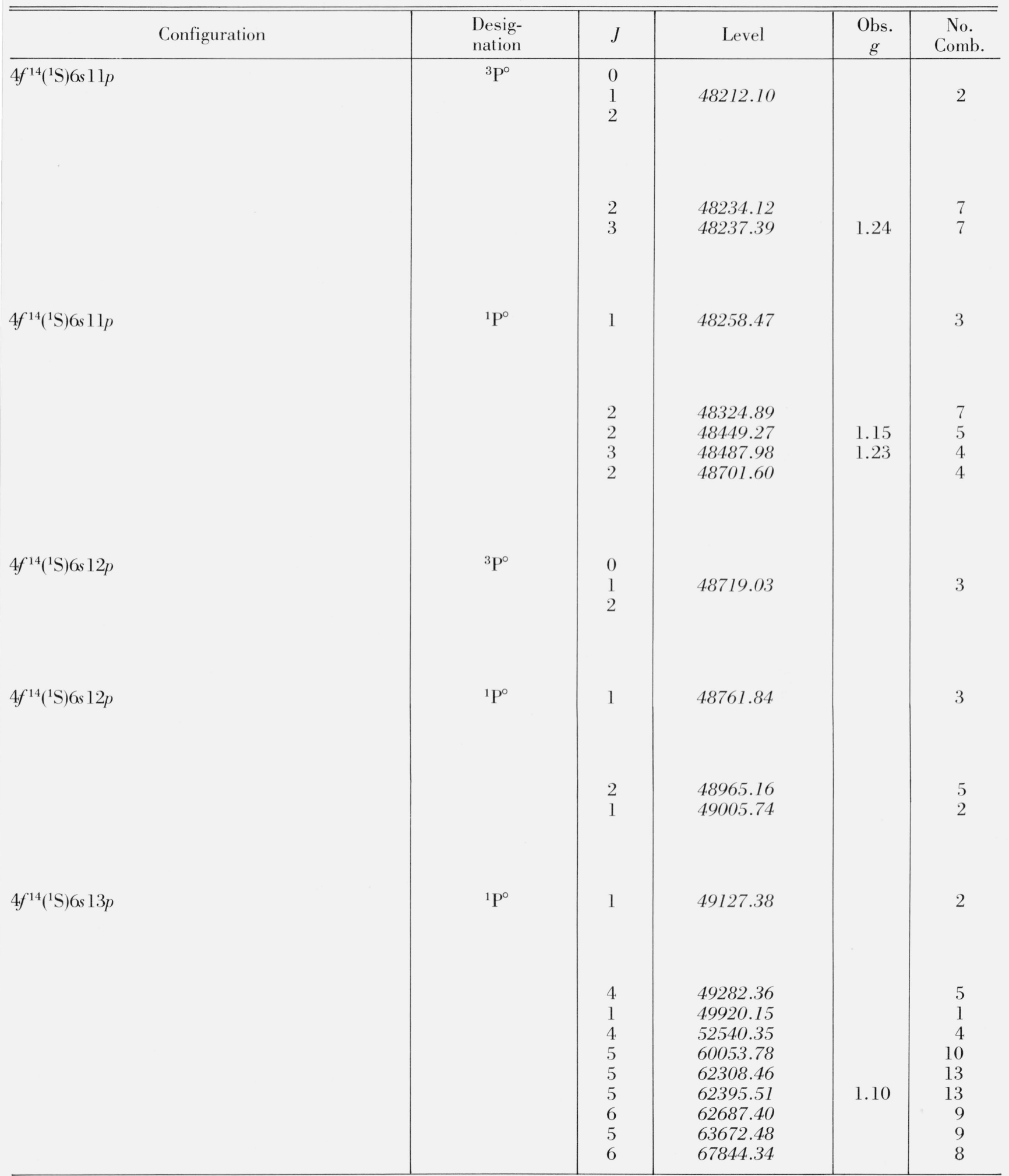




\subsection{Observed Lines and Classifications}

The list of $\mathrm{Yb}$ I lines in table 4 is taken from Meggers and Corliss [1966] in the region 2155 to $11604 \AA$ and from Humphreys and Paul [1959] in the region 12000 to 25000 $\AA$. The three lines at longer wavelengths were reported privately to Meggers by Humphreys and Paul in 1964.

The wavelengths in air appear in the first column of table 4. Estimated intensities as observed in the electrodeless microwave discharge lamp (Meggers lamp) and in the ring discharge (Thomson lamp) appear in the next two columns. Literal symbols in the intensity column have the following meanings:

A - nearly all absorbed in the pulsed arc

$\mathrm{R}$ - wide reversal in the pulsed arc

$\mathrm{r}$ - narrow reversal in the pulsed arc

$\mathrm{d}$-double but not resolved

w - wide

h - hazy

$l$ - shaded to longer wavelengths

$\mathrm{s}$ - shaded to shorter wavelengths

Z - Zeeman pattern observed

The wavenumbers are in column 3 followed by the classification in column 4 . The first entry in this column is the lower energy level and its $J$-value, which is followed by the upper level with its $J$-value. The odd levels are distinguished from the even ones by a superscript degree symbol. The difference between the observed wavenumber of the line and its value calculated from the two energy levels $(\mathrm{O}-\mathrm{C})$ appears in the last column in units of $0.01 \mathrm{~cm}^{-1}$.

We wish to acknowledge with sincere thanks the contribution to this work by Charlotte E. Moore, who furnished the collation of Meggers' original material and completed his derivation of $g$-values. We are also most grateful for the cordial cooperation of Mrs. Z. B. Goldschmidt for providing us some of the results of her theoretical calculations in advance of publication.

Dr. Meggers especially appreciated the early contributions of N. Spector [1971] to the interpretation of this spectrum.

We would like to thank C. H. Corliss for many helpful discussions and for the photographs of ytterbium spectra reproduced here in figure 1.

Finally, we acknowledge the continuing interest of W. C. Martin and R. Zalubas in this spectrum and the illuminating discussions with them on its interpretation. They were also very generous in helping to get the manuscript to press.

\section{References}

Baumann, M., and Wandel, G. [1968], Phys. Lett. 28A, 200.

Budick, B., and Snir, J. [1967], Phys. Lett. 24A, 689.

Camus, P., and Tomkins, F. S. [1969], J. Phys. (Paris) 30, 545.

Corliss, C. H., and Meggers, W. F. [1958], J. Res. Nat. Bur. Stand (U.S.), 61, No. 4, 269-272 (Oct. 1958) RP 2904.

Humphreys, C. J., and Paul, E. [1959], Infrared Atomic Spectra, Quarterly Report Foundational Research Projects NAVORD Report 5970, NOLC Report 473, 57 pp.

Kaufman, V., and Sugar, J. [1973], J. Opt. Soc. Am. 63, 1168.

King, A. S. [1931], Astrophys. J. 74, 328.

Meggers, W. F. (C. E. Moore, Editor), [1967], J. Res Nat. Bur. Stand. (U.S.), 71A, No. 6, 396-544 (Nov.-Dec. 1967).

Meggers, W. F. and Corliss, C. H. [1960], J. Opt. Soc. Am. 50, 1136 A.

Meggers, W. F. and Corliss, C. H. [1966], J. Res. Nat. Bur. Stand. (U.S.), 70A, No. 1, 63-106 (Jan.-Feb. 1966).

Meggers, W. F., Corliss, C. H. and Scribner, B. F. [1975], Tables of Spectral-Line Intensities, NBS Mono. 145, Pt. I (U.S. Gov't Printing Office, Washington, D.C.).

Meggers, W. F., and Scribner, B. F. [1937], J. Res. Nat. Bur. Stand (U.S.), 19, 651-664 (Dec. 1937)RP1053.

Miller, G. E., and Ross, J. S. [1976], J. Opt. Soc. Am. 66, 585.

Nir, S. [1970], J. Opt. Soc. Am. 60, 354.

Parr, A. C. and Elder, F. A. [1968], J. Chem. Phys. 49, 2665.

Reader, J. and Sugar, J. [1966], J. Opt. Soc. Am. 56, 1189.

Spector, N. [1971], J. Opt. Soc. Am. 61, 1350.

Sugar, J. [1962], J. Res. Nat. Bur. Stand. (U.S.), 66A, No. 4, 321-324 (July-Aug. 1962) 
TABLE 4. Observed and classified lines of $\mathrm{Yb}$ I

\begin{tabular}{|c|c|c|c|c|c|}
\hline \multirow{2}{*}{$\begin{array}{c}\text { Wavelength } \\
\text { (air) } \\
\AA\end{array}$} & \multicolumn{2}{|c|}{ Intensity } & \multirow{2}{*}{$\underset{\substack{\text { Wavenumber } \\
\mathrm{cm}^{-1}}}{\text { W }}$} & \multirow[b]{2}{*}{ Classification } & \multirow{2}{*}{$\begin{array}{c}\mathrm{O}-\mathrm{C} \\
0.01 \mathrm{~cm}^{-1}\end{array}$} \\
\hline & $\begin{array}{l}\text { Meggers } \\
\text { Lamp }\end{array}$ & $\begin{array}{l}\text { Thomson } \\
\text { Lamp }\end{array}$ & & & \\
\hline 31308.3 & 3 & & 3193.17 & $44453_{3}^{\circ}-476463$ & +2 \\
\hline 29813.2 & 4 & & 3353.30 & & \\
\hline 28896.7 & 2 & & 3459.66 & & \\
\hline 24552.51 & 10 & & 4071.79 & $34350_{0}-38422_{1}^{\circ}$ & +8 \\
\hline 24448.31 & 45 & & 4089.15 & $28184_{4}^{\circ}-32273_{4}$ & +6 \\
\hline 23117.83 & 12 & & 4324.48 & & \\
\hline 22592.6 & 400 & & 4425.02 & & \\
\hline 21640.70 & 100 & & 4619.66 & $27445_{3}^{\circ}-32065_{3}$ & +2 \\
\hline 21480.74 & 80 & & 4654.06 & $30524 \stackrel{\circ}{-}-351785$ & +0 \\
\hline 21180.98 & 10 & & 4719.93 & $326941-37414_{1}^{\circ}$ & +3 \\
\hline 20920.26 & 85 & & 4778.75 & $19710_{2}^{\circ}-24489_{1}$ & +4 \\
\hline 20706.52 & 38 & & 4828.08 & $27445_{3}^{\circ}-322734$ & +12 \\
\hline 20036.01 & 42 & & 4989.65 & $30207_{3}^{\circ}-35196_{2}$ & +5 \\
\hline 19829.77 & 3600 & & 5041.55 & $19710_{2}^{\circ}-24751_{2}$ & -1 \\
\hline 19776.44 & 20 & & 5055.14 & & \\
\hline 19452.96 & 10 & & 5139.20 & & \\
\hline 18526.88 & 45 & & 5396.09 & $326941-38090_{0}^{\circ}$ & +7 \\
\hline 18244.0 & 1000 & & 5479.76 & $326941-38174_{1}^{\circ}$ & +27 \\
\hline 18196.8 & 200 & & 5493.97 & & \\
\hline 18057.11 & 250 & & 5536.47 & & \\
\hline 17979.09 & 2500 & & 5560.50 & $19710_{2}^{\circ}-25270_{3}$ & -2 \\
\hline 17851.79 & 400 & & 5600.15 & $30207_{3}^{\circ}-35807_{3}$ & +1 \\
\hline 17454.72 & 175 & & 5727.55 & $326941-38422_{1}^{\circ}$ & -12 \\
\hline 17151.32 & 20 & & 5828.86 & $38551_{2}^{\circ}-44380_{3}$ & -3 \\
\hline 17078.60 & 35 & & 5853.68 & $30207_{3}^{\circ}-36060_{4}$ & +8 \\
\hline 17068.36 & 850 & & 5857.19 & $32694_{1}-38551_{2}^{\circ}$ & -4 \\
\hline 16571.55 & 700 & & 6032.79 & & \\
\hline 16089.88 & 185 & & 6213.39 & $34350 \mathrm{o}-40563^{\circ}$ & +7 \\
\hline 15903.98 & 550 & & 6286.02 & $29774_{4}^{\circ}-36060_{4}$ & +0 \\
\hline 15768.68 & 200 & & 6339.95 & $28857_{1}^{\circ}-351962$ & -1 \\
\hline 15586.88 & 3500 & & 6413.90 & $258599^{\circ}-32273_{4}$ & -1 \\
\hline 15387.34 & 1250 & & 6497.07 & $17992_{1}^{\circ}-244891$ & -2 \\
\hline 15308.15 & 35 & & 6530.68 & & \\
\hline 14789.02 & 30000 & & 6759.93 & $17992_{1}^{\circ}-24751_{2}$ & -1 \\
\hline 14293.67 & 200 & & 6994.19 & $28184_{4}^{\circ}-351785$ & -8 \\
\hline 14279.64 & 700 & & 7001.06 & $28195_{2}^{\circ}-351962$ & +4 \\
\hline 13883.85 & 20000 & & 7200.64 & $17288 \circ-24489_{1}$ & -2 \\
\hline 13689.96 & 17 & & 7302.63 & & \\
\hline 13134.21 & 275 & & 7611.62 & $28195_{2}^{\circ}-35807_{3}$ & +6 \\
\hline 13119.05 & 3 & & 7620.42 & & \\
\hline 13114.42 & 35 & & 7623.11 & $28184_{4}^{\circ}-35807_{3}$ & +10 \\
\hline 13108.27 & 42 & & 7626.69 & & \\
\hline 12712.91 & 4500 & & 7863.87 & $27314_{6}^{\circ}-351785$ & +1 \\
\hline 12692.48 & 450 & & 7876.53 & $28184_{4}^{\circ}-36060_{4}$ & +6 \\
\hline 12547.90 & 100 & & 7967.28 & $19710_{2}^{\circ}-27677_{2}$ & +0 \\
\hline
\end{tabular}


TABLE 4. Observed and classified lines of $\mathrm{Yb} \mathrm{I}$-Continued

\begin{tabular}{|c|c|c|c|c|c|}
\hline \multirow{2}{*}{$\begin{array}{c}\text { Wavelength } \\
\text { (air) } \\
\AA\end{array}$} & \multicolumn{2}{|c|}{ Intensity } & \multirow{2}{*}{ 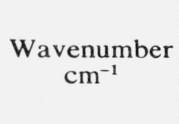 } & \multirow[b]{2}{*}{ Classification } & \multirow{2}{*}{$\begin{array}{c}\mathrm{O}-\mathrm{C} \\
0.01 \mathrm{~cm}^{-1}\end{array}$} \\
\hline & $\begin{array}{c}\text { Meggers } \\
\text { Lamp }\end{array}$ & $\begin{array}{c}\text { Thomson } \\
\text { Lamp }\end{array}$ & & & \\
\hline 11603.94 & 425 & & 8615.40 & $27445_{3}^{\circ}-36060_{4}$ & +6 \\
\hline 11262.27 & 5250 & 10 & 8876.77 & $23188_{2}^{\circ}-32065_{3}$ & +1 \\
\hline 10992.33 & $2 h$ & & 9094.76 & $38551_{2}^{\circ}-476463$ & +7 \\
\hline 10770.10 & 3750 & 400 & 9282.42 & $25068^{\circ}-34350_{0}$ & +0 \\
\hline 10727.72 & 200 & 20 & 9319.09 & $258595-351785$ & -1 \\
\hline 10717.02 & 2 & & 9328.40 & & \\
\hline 10633.24 & 5 & & 9401.90 & & \\
\hline 10567.5 & 1 & & 9460.38 & $38174_{1}^{\circ}-476342$ & +14 \\
\hline 10516.61 & 60 & & 9506.16 & $23188_{2}^{\circ}-32694_{1}$ & -1 \\
\hline 10397.88 & 4 & & 9614.71 & & \\
\hline 10343.85 & 3 & & 9664.93 & & \\
\hline 10341.77 & 7 & & 9666.88 & $34350_{0}-44017_{1}^{\circ}$ & -7 \\
\hline 10321.68 & 500 & 80 & 9685.69 & $17992_{1}^{\circ}-27677_{2}$ & +3 \\
\hline 10267.37 & 200 & 100 & 9736.92 & $27677_{2}-37414_{1}^{\circ}$ & +0 \\
\hline 10212.27 & 1 & & 9789.46 & & \\
\hline 10110.87 & 10 & & 9887.64 & & \\
\hline 9970.44 & 5 & & 10026.90 & & \\
\hline 9894.20 & 8 & & 10104.16 & & \\
\hline 9888.40 & 2 & & 10110.09 & & \\
\hline 9882.33 & 1 & & 10116.30 & & \\
\hline 9870.17 & 200 & 15 & 10128.76 & $25068_{1}^{\circ}-35196_{2}$ & +1 \\
\hline 9831.13 & 7 & & 10168.98 & & \\
\hline 9826.14 & 1 & & 10174.15 & & \\
\hline 9823.76 & 9 & 2 & 10176.61 & & \\
\hline 9817.16 & 1 & & 10183.45 & $38174_{1}^{\circ}-48357_{1}$ & +8 \\
\hline 9799.96 & 400 & 10 & 10201.33 & $25859_{5}^{\circ}-36060_{4}$ & +3 \\
\hline 9775.49 & 1 & & 10226.86 & & \\
\hline 9734.62 & 2 & & 10269.80 & $38090_{0}^{\circ}-48360_{1}$ & +8 \\
\hline 9718.81 & 3 & & 10286.50 & $38551_{2}^{\circ}-488381$ & +10 \\
\hline 9695.71 & 2 & & 10311.01 & & \\
\hline 9688.77 & 25 & & 10318.40 & & \\
\hline 9670.80 & 5 & & 10337.57 & & \\
\hline 9659.43 & 2 & & 10349.74 & & \\
\hline 9656.05 & 20 & & 10353.36 & & \\
\hline 9640.78 & 2 & & 10369.76 & & \\
\hline 9606.08 & 3 & & 10407.22 & $37414_{1}^{\circ}-47821_{2}$ & +3 \\
\hline 9592.23 & 1 & & 10422.25 & & \\
\hline 9589.34 & 1 & & 10425.39 & & \\
\hline 9580.31 & 30 & & 10435.21 & & \\
\hline 9572.65 & 5 & & 10443.56 & $35807_{3}-46251_{3}^{\circ}$ & +2 \\
\hline 9532.64 & 1 & & 10487.40 & & \\
\hline 9524.36 & 300 & 6 & 10496.51 & $27677_{2}-38174_{1}^{\circ}$ & +1 \\
\hline 9512.32 & 2 & & 10509.80 & & \\
\hline 9505.71 & 2 & & 10517.11 & & \\
\hline 9468.54 & 2 & 3 & 10558.39 & & \\
\hline
\end{tabular}


TABLE 4. Observed and classified lines of $\mathrm{Yb} \mathrm{I}$-Continued

\begin{tabular}{|c|c|c|c|c|c|}
\hline \multirow{2}{*}{$\begin{array}{c}\text { Wavelength } \\
\text { (air) } \\
\AA\end{array}$} & \multicolumn{2}{|c|}{ Intensity } & \multirow{2}{*}{$\underset{\substack{\mathrm{cm}^{-1} \\
\text { Wavenumber }}}{ }$} & \multirow[b]{2}{*}{ Classification } & \multirow{2}{*}{$\begin{array}{c}\mathrm{O}-\mathrm{C} \\
0.01 \mathrm{~cm}^{-1}\end{array}$} \\
\hline & $\begin{array}{l}\text { Meggers } \\
\text { Lamp }\end{array}$ & $\begin{array}{l}\text { Thomson } \\
\text { Lamp }\end{array}$ & & & \\
\hline 9382.31 & 2 & & 10655.43 & & \\
\hline 9377.90 & 7 & & 10660.44 & $32065_{3}-42725_{2}^{\circ}$ & -3 \\
\hline 9304.35 & 1000 & 80 & 10744.71 & $27677_{2}-38422_{1}^{\circ}$ & +2 \\
\hline 9298.90 & 3 & & 10751.01 & & \\
\hline 9291.74 & 2 & & 10759.29 & $351962-45956_{2}^{\circ}$ & +1 \\
\hline 9258.76 & 1 & & 10797.62 & $528746-63672 \stackrel{\circ}{\circ}$ & -7 \\
\hline 9230.17 & 7 & & 10831.06 & $34350_{0}-45181_{1}^{\circ}$ & +2 \\
\hline 9224.21 & 6 & & 10838.06 & $326941-43532_{1}^{\circ}$ & -2 \\
\hline 9188.34 & 10 & & 10880.37 & & \\
\hline 9177.47 & 1 & & 10893.26 & & \\
\hline 9176.14 & 1 & & 10894.84 & $37414_{1}^{\circ}-48309_{1}$ & +2 \\
\hline 9155.30 & 40 & & 10919.64 & $326941-43614_{0}^{\circ}$ & +6 \\
\hline 9140.64 & 10 & & 10937.15 & & \\
\hline 9128.47 & 20 & & 10951.73 & $28857_{1}^{\circ}-39808_{1}$ & +3 \\
\hline 9121.11 & 3 & & 10960.57 & & \\
\hline 9117.68 & 100 & 4 & 10964.69 & $32694_{1}-43659_{1}^{\circ}$ & +1 \\
\hline 9104.10 & 200 & 7 & 10981.05 & $28857_{1}^{\circ}-39838_{2}$ & +2 \\
\hline 9097.69 & 2 & & 10988.79 & & \\
\hline 9089.55 & $2 d$ & & 10998.63 & & \\
\hline 9073.18 & 1 & & 11018.47 & & \\
\hline 9069.22 & 20 & & 11023.28 & $28857_{1}^{\circ}-39880_{2}$ & +4 \\
\hline 9045.47 & 1 & & 11052.22 & $48324_{2}^{\circ}-59377_{2}$ & -2 \\
\hline 9043.95 & 5 & & 11054.08 & $351962-46251_{3}^{\circ}$ & +0 \\
\hline 9039.08 & 1 & & 11060.04 & & \\
\hline 9020.90 & 10 & & 11082.33 & & \\
\hline 9016.21 & 2 & & 11088.09 & & \\
\hline 8997.66 & 400 & 10 & 11110.95 & $32694_{1}-43805_{2}^{\circ}$ & -5 \\
\hline 8980.79 & 3 & & 11131.82 & & \\
\hline 8948.02 & 2 & & 11172.59 & & \\
\hline 8946.38 & 1 & & 11174.64 & & \\
\hline 8922.50 & 1000 & 40 & 11204.54 & $28857_{1}^{\circ}-40061_{2}$ & +5 \\
\hline 8902.99 & 2 & & 11229.10 & & \\
\hline 8898.89 & 1 & & 11234.27 & & \\
\hline 8888.66 & 3 & & 11247.20 & $488066-600535$ & -1 \\
\hline 8883.16 & 10 & & 11254.17 & & \\
\hline 8874.68 & 2 & & 11264.92 & & \\
\hline 8864.06 & 2 & & 11278.42 & & \\
\hline 8831.38 & 2 & & 11320.15 & & \\
\hline 8829.26 & 30 & & 11322.87 & $32694_{1}-44017_{1}^{\circ}$ & -4 \\
\hline 8827.46 & 1 & & 11325.18 & & \\
\hline 8806.80 & 6 & & 11351.74 & & \\
\hline 8794.91 & 5 & & 11367.09 & & \\
\hline 8788.45 & 5 & & 11375.45 & & \\
\hline 8783.75 & 4 & & 11381.53 & & \\
\hline 8780.63 & 10 & & 11385.58 & & \\
\hline
\end{tabular}


TABLE 4. Observed and classified lines of $\mathrm{Yb} \mathrm{I}$-Continued

\begin{tabular}{|c|c|c|c|c|c|}
\hline \multirow{2}{*}{$\begin{array}{l}\text { Wavelength } \\
\text { (air) } \\
\AA\end{array}$} & \multicolumn{2}{|c|}{ Intensity } & \multirow{2}{*}{$\underset{\mathrm{cm}^{-1}}{\text { Wavenumber }}$} & \multirow[b]{2}{*}{ Classification } & \multirow{2}{*}{$\begin{array}{c}\mathrm{O}-\mathrm{C} \\
0.01 \mathrm{~cm}^{-1}\end{array}$} \\
\hline & $\begin{array}{c}\text { Meggers } \\
\text { Lamp }\end{array}$ & $\begin{array}{c}\text { Thomson } \\
\text { Lamp }\end{array}$ & & & \\
\hline 8736.45 & 20 & & 11443.15 & & \\
\hline 8736.03 & 4 & & 11443.70 & & \\
\hline 8731.29 & 30 & & 11449.92 & & \\
\hline 8724.18 & 4 & & 11459.25 & & \\
\hline 8717.80 & 1 & & 11467.63 & & \\
\hline 8686.28 & 2 & & 11509.25 & & \\
\hline 8681.93 & 3 & & 11515.01 & & \\
\hline 8679.50 & 2 & & 11518.24 & & \\
\hline 8670.82 & 2 & & 11529.77 & & \\
\hline 8667.69 & 50 & & 11533.93 & $47843_{3}^{\circ}-59377_{2}$ & -7 \\
\hline 8663.92 & 10 & & 11538.95 & & \\
\hline 8661.59 & 5 & & 11542.05 & $32273_{4}-43815_{3}^{\circ}$ & -2 \\
\hline 8655.52 & $15 h$ & & 11550.15 & & \\
\hline 8654.91 & $100 w$ & & 11550.96 & & \\
\hline 8654.42 & 20 & & 11551.62 & & \\
\hline 8650.23 & 2 & & 11557.21 & $326941-44251_{2}^{\circ}$ & +2 \\
\hline 8634.68 & 9 & & 11578.03 & & \\
\hline 8612.57 & 50 & & 11607.75 & & \\
\hline 8603.56 & 80 & & 11619.90 & $30207_{3}^{\circ}-41827_{3}$ & -2 \\
\hline 8601.25 & 1 & & 11623.02 & & \\
\hline 8594.75 & 2 & & 11631.81 & & \\
\hline 8592.00 & 200 & & 11635.54 & & \\
\hline 8591.26 & 20 & & 11636.54 & & \\
\hline 8590.94 & 20 & & 11636.97 & & \\
\hline 8590.08 & 1 & & 11638.14 & & \\
\hline 8585.99 & 20 & & 11643.68 & & \\
\hline 8584.20 & 2 & & 11646.11 & & \\
\hline 8575.25 & 10 & & 11658.27 & & \\
\hline 8574.91 & 2 & & 11658.73 & & \\
\hline 8558.47 & $2 h$ & & 11681.12 & & \\
\hline 8546.26 & 40 & & 11697.81 & & \\
\hline 8535.68 & 20 & & 11712.31 & & \\
\hline 8528.08 & 10 & & 11722.75 & & \\
\hline 8525.66 & 3 & & 11726.07 & & \\
\hline 8524.07 & 3 & & 11728.26 & $34350 \mathrm{o}-46078^{\circ}$ & +0 \\
\hline 8522.92 & 2 & & 11729.84 & & \\
\hline 8520.34 & 50 & & 11733.40 & & \\
\hline 8520.12 & 7 & & 11733.70 & & \\
\hline 8519.66 & 20 & & 11734.33 & & \\
\hline 8518.01 & 20 & & 11736.61 & & \\
\hline 8516.60 & 2 & & 11738.55 & & \\
\hline 8515.78 & 3 & & 11739.68 & & \\
\hline 8515.24 & 2 & & 11740.42 & $32065_{3}-43805_{2}^{\circ}$ & +2 \\
\hline 8508.02 & 10 & & 11750.39 & $320653-43815_{3}^{\circ}$ & +0 \\
\hline 8505.96 & 2 & & 11753.23 & & \\
\hline
\end{tabular}


TABLE 4. Observed and classified lines of $\mathrm{Yb} \mathrm{I}$-Continued

\begin{tabular}{|c|c|c|c|c|c|}
\hline \multirow{2}{*}{$\begin{array}{c}\text { Wavelength } \\
\text { (air) } \\
\AA\end{array}$} & \multicolumn{2}{|c|}{ Intensity } & \multirow{2}{*}{$\underset{\mathrm{cm}^{-1}}{\text { Wavenumber }}$} & \multirow[b]{2}{*}{ Classification } & \multirow{2}{*}{$\begin{array}{c}\mathrm{O}-\mathrm{C} \\
0.01 \mathrm{~cm}^{-1}\end{array}$} \\
\hline & $\begin{array}{l}\text { Meggers } \\
\text { Lamp }\end{array}$ & $\begin{array}{l}\text { Thomson } \\
\text { Lamp }\end{array}$ & & & \\
\hline 8494.37 & 50 & & 11769.27 & & \\
\hline 8493.74 & 4 & & 11770.14 & $28195_{2}^{\circ}-39966_{3}$ & +1 \\
\hline 8487.84 & 1 & & 11778.32 & & \\
\hline 8485.10 & 30 & & 11782.13 & $36060_{4}-47843_{3}^{\circ}$ & -2 \\
\hline 8474.21 & 3 & & 11797.27 & & \\
\hline 8463.32 & 1 & & 11812.45 & & \\
\hline 8462.25 & 4 & & 11813.94 & & \\
\hline 8446.74 & 5 & & 11835.64 & & \\
\hline 8446.35 & 20 & & 11836.18 & & \\
\hline 8443.99 & 5 & & 11839.49 & & \\
\hline 8430.76 & 2 & & 11858.07 & $35196_{2}-47055_{2}^{\circ}$ & +0 \\
\hline 8429.59 & 3 & & 11859.71 & & \\
\hline 8428.24 & 10 & & 11861.61 & & \\
\hline 8423.84 & 1 & & 11867.81 & & \\
\hline 8418.43 & 10 & & 11875.44 & & \\
\hline 8416.53 & 20 & & 11878.12 & $36060_{4}-47939_{4}^{\circ}$ & +0 \\
\hline 8400.65 & 20 & & 11900.57 & & \\
\hline 8400.35 & 5 & & 11900.99 & & \\
\hline 8391.62 & 3 & & 11913.38 & & \\
\hline 8383.90 & 2 & & 11924.35 & & \\
\hline 8378.93 & 6 & & 11931.42 & & \\
\hline 8376.33 & 2 & & 11935.12 & & \\
\hline 8375.94 & 40 & & 11935.68 & & \\
\hline 8360.09 & 2 & & 11958.31 & & \\
\hline 8352.03 & 2 & & 11969.85 & & \\
\hline 8349.52 & $2 h$ & & 11973.45 & & \\
\hline 8346.11 & $3 h$ & & 11978.34 & & \\
\hline 8335.12 & 1 & & 11994.13 & & \\
\hline 8333.30 & 10 & & 11996.75 & & \\
\hline 8327.71 & 3 & & 12004.80 & & \\
\hline 8325.18 & 800 & $5 Z$ & 12008.45 & $23188_{2}^{\circ}-351962$ & -1 \\
\hline 8320.33 & 8 & & 12015.45 & & \\
\hline 8317.43 & 20 & & 12019.64 & $34350_{0}-46370_{1}^{\circ}$ & -1 \\
\hline 8310.61 & 3 & & 12029.51 & & \\
\hline 8309.10 & 2 & & 12031.69 & & \\
\hline 8306.38 & 2 & & 12035.63 & $35807_{3}-47843_{3}^{\circ}$ & +2 \\
\hline 8299.77 & 2 & & 12045.22 & & \\
\hline 8296.83 & 4 & & 12049.48 & & \\
\hline 8294.85 & 150 & & 12052.36 & $29774_{4}^{\circ}-41827_{3}$ & +2 \\
\hline 8267.87 & 10 & & 12091.69 & & \\
\hline 8266.06 & 2 & & 12094.34 & & \\
\hline 8265.64 & 2 & & 12094.95 & & \\
\hline 8259.34 & 1 & & 12104.18 & & \\
\hline 8249.18 & 50 & & 12119.09 & $322734-44392_{4}^{\circ}$ & -2 \\
\hline 8242.95 & 1 & & 12128.25 & $351962-47325^{\circ}$ & +4 \\
\hline
\end{tabular}


TABLE 4. Observed and classified lines of $\mathrm{Yb} \mathrm{I}$-Continued

\begin{tabular}{|c|c|c|c|c|c|}
\hline \multirow{2}{*}{$\begin{array}{c}\text { Wavelength } \\
\text { (air) } \\
\AA\end{array}$} & \multicolumn{2}{|c|}{ Intensity } & \multirow{2}{*}{$\underset{\mathrm{cm}^{-1}}{\text { Wavenumber }}$} & \multirow[b]{2}{*}{ Classification } & \multirow{2}{*}{$\begin{array}{c}\mathrm{O}-\mathrm{C} \\
0.01 \mathrm{~cm}^{-1}\end{array}$} \\
\hline & $\begin{array}{l}\text { Meggers } \\
\text { Lamp }\end{array}$ & $\begin{array}{l}\text { Thomson } \\
\text { Lamp }\end{array}$ & & & \\
\hline 8242.03 & 1 & & 12129.60 & $351962-47326_{2}^{\circ}$ & -7 \\
\hline 8240.67 & 2 & & 12131.60 & $35807_{3}-47939_{4}^{\circ}$ & +2 \\
\hline 8234.61 & 15 & & 12140.53 & & \\
\hline 8225.59 & $1 h$ & & 12153.84 & & \\
\hline 8224.13 & 10 & & 12156.00 & & \\
\hline 8221.74 & 10 & & 12159.53 & & \\
\hline 8221.35 & 10 & & 12160.11 & & \\
\hline 8220.44 & 2 & & 12161.46 & & \\
\hline 8213.96 & 9 & & 12171.05 & & \\
\hline 8213.02 & 30 & & 12172.44 & & \\
\hline 8212.03 & 5 & & 12173.91 & & \\
\hline 8210.33 & 4 & & 12176.43 & $36060_{4}-48237_{3}^{\circ}$ & +2 \\
\hline 8207.98 & 40 & & 12179.92 & $322734-44453_{3}^{\circ}$ & +4 \\
\hline 8200.20 & 1 & & 12191.47 & & \\
\hline 8197.43 & 6 & & 12195.59 & & \\
\hline 8190.31 & 5 & & 12206.19 & & \\
\hline 8180.75 & 10 & & 12220.46 & & \\
\hline 8180.64 & 30 & & 12220.62 & & \\
\hline 8145.56 & 20 & & 12273.25 & & \\
\hline 8145.36 & 5 & & 12273.55 & & \\
\hline 8140.53 & 2 & & 12280.84 & $477724-600535$ & +0 \\
\hline 8136.38 & 9 & & 12287.10 & & \\
\hline 8122.77 & 1 & & 12307.69 & & \\
\hline 8116.08 & 10 & & 12317.83 & & \\
\hline 8114.26 & 9 & & 12320.59 & & \\
\hline 8111.71 & 90 & & 12324.47 & $30207_{3}^{\circ}-425312$ & -2 \\
\hline 8109.79 & 100 & & 12327.39 & $32065_{3}-44392_{4}^{\circ}$ & -3 \\
\hline 8098.66 & 2 & & 12344.33 & & \\
\hline 8095.74 & 1 & & 12348.78 & & \\
\hline 8092.95 & 3 & & 12353.04 & & \\
\hline 8091.73 & 1000 & $10 Z$ & 12354.90 & $19710_{2}^{\circ}-32065_{3}$ & +1 \\
\hline 8089.66 & 7 & & 12358.06 & & \\
\hline 8086.68 & 10 & & 12362.61 & & \\
\hline 8085.56 & 4 & & 12364.33 & & \\
\hline 8084.50 & 1 & & 12365.95 & & \\
\hline 8071.28 & 10 & & 12386.20 & & \\
\hline 8070.00 & 50 & & 12388.17 & $32065_{3}-44453_{3}^{\circ}$ & -2 \\
\hline 8067.21 & 7 & & 12392.45 & $27445_{3}^{\circ}-39838_{2}$ & +5 \\
\hline 8066.43 & 7 & & 12393.65 & & \\
\hline 8058.91 & 80 & & 12405.21 & & \\
\hline 8056.20 & 10 & & 12409.39 & & \\
\hline 8055.12 & 20 & & 12411.05 & $30524_{5}^{\circ}-42935_{5}$ & -1 \\
\hline 8052.35 & 20 & & 12415.32 & & \\
\hline 8051.48 & 20 & & 12416.66 & & \\
\hline 8042.91 & 4 & & 12429.89 & $35807_{3}-48237_{3}^{\circ}$ & +2 \\
\hline
\end{tabular}


TABLE 4. Observed and classified lines of $\mathrm{Yb} \mathrm{I}-$ Continued

\begin{tabular}{|c|c|c|c|c|c|}
\hline \multirow{2}{*}{$\begin{array}{c}\text { Wavelength } \\
\text { (air) } \\
\AA\end{array}$} & \multicolumn{2}{|c|}{ Intensity } & \multirow{2}{*}{$\underset{\mathrm{cm}^{-1}}{\text { Wavenumber }}$} & \multirow{2}{*}{ Classification } & \multirow{2}{*}{$\begin{array}{c}\mathrm{O}-\mathrm{C} \\
0.01 \mathrm{~cm}^{-1}\end{array}$} \\
\hline & $\begin{array}{l}\text { Meggers } \\
\text { Lamp }\end{array}$ & $\begin{array}{l}\text { Thomson } \\
\text { Lamp }\end{array}$ & & & \\
\hline 8039.83 & 200 & \multirow[t]{5}{*}{$2 Z$} & 12434.65 & $27445_{3}^{\circ}-39880_{2}$ & +3 \\
\hline 8023.11 & 3 & & 12460.57 & $32694_{1}-45155_{2}^{\circ}$ & -7 \\
\hline 8018.74 & $3 h$ & & 12467.36 & & \\
\hline 7990.35 & 30 & & 12511.66 & & \\
\hline 7946.26 & 50 & & 12581.08 & & \\
\hline 7933.25 & 2 & \multirow{5}{*}{$100 Z$} & 12601.71 & & \\
\hline 7924.65 & 4 & & 12615.38 & & \\
\hline 7922.40 & 4000 & & 12618.97 & \multirow{3}{*}{$23188_{2}^{\circ}-35807_{3}$} & \multirow{3}{*}{-4} \\
\hline 7910.08 & 10 & & 12638.62 & & \\
\hline 7896.38 & 100 & & 12660.55 & & \\
\hline 7895.08 & 1000 & \multirow[t]{4}{*}{$50 Z$} & 12662.63 & $24751_{2}-37414_{1}^{\circ}$ & -1 \\
\hline 7883.96 & 2 & & 12680.49 & $35807_{3}-48487_{3}^{\circ}$ & \multirow{4}{*}{+3} \\
\hline 7877.06 & 60 & & 12691.60 & & \\
\hline 7875.15 & 10 & & 12694.68 & & \\
\hline 7844.11 & 20 & \multirow[t]{9}{*}{2} & 12744.91 & & \\
\hline 7834.65 & 15 & & 12760.30 & $351785-47939_{4}^{\circ}$ & \multirow[t]{8}{*}{-2} \\
\hline 7821.37 & 2 & & 12781.97 & & \\
\hline 7820.89 & 50 & & 12782.75 & & \\
\hline 7799.33 & 1 & & 12818.09 & & \\
\hline 7789.80 & 2 & & 12833.77 & & \\
\hline 7771.01 & 4 & & 12864.80 & & \\
\hline 7761.45 & 2 & & 12880.65 & & \\
\hline 7760.49 & 5 & & 12882.24 & & \\
\hline 7758.04 & 500 & \multirow[t]{2}{*}{$20 Z$} & 12886.31 & $27677_{2}-40563_{1}^{\circ}$ & \multirow[t]{2}{*}{+0} \\
\hline 7746.98 & $6 h$ & & 12904.70 & & \\
\hline 7744.97 & 5 & \multirow{4}{*}{$2 Z$} & 12908.05 & & \\
\hline 7734.53 & 200 & & 12925.48 & \multirow[t]{3}{*}{$24489_{1}-37414_{1}^{\circ}$} & \multirow[t]{3}{*}{-1} \\
\hline 7717.58 & 6 & & 12953.86 & & \\
\hline 7706.29 & $2 h$ & & 12972.84 & & \\
\hline 7699.49 & 20000 & $2000 Z$ & 12984.30 & $19710_{2}^{\circ}-326941$ & +0 \\
\hline 7692.75 & $5 h$ & \multirow{15}{*}{$1 Z$} & 12995.68 & & \\
\hline 7679.91 & 200 & & 13017.40 & $30207_{3}^{\circ}-43224_{2}$ & +0 \\
\hline 7662.08 & 2 & & 13047.69 & \multirow[t]{5}{*}{$49260_{4}-62308_{5}^{\circ}$} & \multirow[t]{3}{*}{-1} \\
\hline 7659.90 & 20 & & 13051.41 & & \\
\hline 7659.14 & 50 & & 13052.70 & & \\
\hline 7657.60 & 100 & & 13055.33 & & \\
\hline 7650.02 & 4 & & 13068.26 & & \\
\hline 7641.45 & 3 & & 13082.92 & $28857_{1}^{\circ}-419390$ & +3 \\
\hline 7637.27 & 15 & & 13090.08 & $32065_{3}-45155_{2}^{\circ}$ & +3 \\
\hline 7623.90 & 20 & & 13113.04 & & \\
\hline 7616.34 & 3 & & 13126.05 & $\left\{547185-67844_{6}^{\circ}\right.$ & $\{-4$ \\
\hline & & & 15120.00 & $146251_{3}^{\circ}-59377_{2}$ & -2 \\
\hline 7611.28 & 3 & & 13134.78 & $49260_{4}-62395^{\circ}$ & +2 \\
\hline 7603.42 & 2 & & 13148.36 & & \\
\hline 7598.01 & 2 & & 13157.72 & $35807_{3}-48965_{2}^{\circ}$ & +8 \\
\hline
\end{tabular}


TABLE 4. Observed and classified lines of $\mathrm{Yb} \mathrm{I}-$ Continued

\begin{tabular}{|c|c|c|c|c|c|}
\hline \multirow{2}{*}{$\begin{array}{c}\text { Wavelength } \\
\text { (air) } \\
\AA\end{array}$} & \multicolumn{2}{|c|}{ Intensity } & \multirow{2}{*}{$\underset{\substack{\text { Wavenumber } \\
\mathrm{cm}^{-1}}}{\text { Wat }}$} & \multirow{2}{*}{ Classification } & \multirow{2}{*}{$\begin{array}{c}\mathrm{O}-\mathrm{C} \\
0.01 \mathrm{~cm}^{-1}\end{array}$} \\
\hline & $\begin{array}{c}\text { Meggers } \\
\text { Lamp }\end{array}$ & $\begin{array}{l}\text { Thomson } \\
\text { Lamp }\end{array}$ & & & \\
\hline 7596.22 & 200 & 1 & 13160.82 & $29774_{4}^{\circ}-42935_{5}$ & +0 \\
\hline 7588.43 & 8 & & 13174.33 & & \\
\hline 7582.90 & 30 & & 13183.94 & & \\
\hline 7576.41 & 2 & & 13195.23 & & \\
\hline 7561.42 & 4 & & 13221.39 & $36060_{4}-49282_{4}^{\circ}$ & +1 \\
\hline 7547.08 & 5 & & 13246.51 & & \\
\hline 7527.45 & 10000 & $200 Z$ & 13281.05 & $25270_{3}-38551_{2}^{\circ}$ & +3 \\
\hline 7522.73 & 200 & 2 & 13289.39 & $30524_{5}^{\circ}-438146$ & -1 \\
\hline 7507.59 & 5 & & 13316.19 & $490795-62395_{5}^{\circ}$ & +2 \\
\hline 7499.87 & 30 & & 13329.89 & & \\
\hline 7496.33 & 100 & 2 & 13336.19 & & \\
\hline 7478.77 & 4 & & 13367.50 & & \\
\hline 7472.44 & 2 & & 13378.82 & & \\
\hline 7469.44 & 60 & & 13384.20 & $326941-46078^{\circ}$ & -2 \\
\hline 7467.58 & 40 & 30 & 13387.53 & $326941-46082 \circ$ & +5 \\
\hline 7458.79 & $5 h$ & & 13403.31 & & \\
\hline 7448.28 & 6000 & $100 Z$ & 13422.22 & $24751_{2}-38174_{1}^{\circ}$ & +0 \\
\hline 7438.92 & 25 & 1 & 13439.11 & & \\
\hline 7419.22 & 10 & & 13474.79 & $35807_{3}-49282^{\circ}$ & -5 \\
\hline 7416.64 & 40 & & 13479.48 & $326941-46174{ }_{1}^{\circ}$ & -4 \\
\hline 7414.13 & 5 & & 13484.05 & & \\
\hline 7411.17 & 200 & 2 & 13489.43 & $326941-46184_{2}^{\circ}$ & -3 \\
\hline 7405.94 & 6 & & 13498.96 & $465546-60053 \stackrel{\circ}{5}$ & -1 \\
\hline 7404.35 & 10 & & 13501.86 & $488066-623085$ & -3 \\
\hline 7400.67 & 7 & & 13508.57 & $34350_{0}-47859_{1}^{\circ}$ & -9 \\
\hline 7387.69 & 2 & & 13532.30 & & \\
\hline 7382.17 & 3 & & 13542.42 & & \\
\hline 7361.90 & 200 & 1 & 13579.71 & $28857_{1}^{\circ}-424361$ & +2 \\
\hline 7356.92 & 3 & & 13588.90 & $488066-62395_{5}^{\circ}$ & -4 \\
\hline 7350.04 & 6000 & $100 Z$ & 13601.62 & $24489_{1}-38090_{0}^{\circ}$ & +1 \\
\hline 7346.60 & 3 & & 13607.99 & $490795-62687_{6}^{\circ}$ & -7 \\
\hline 7339.99 & 10 & & 13620.24 & $486886-623085$ & -4 \\
\hline 7334.01 & 50 & & 13631.35 & $28195_{2}^{\circ}-41827_{3}$ & +1 \\
\hline 7328.57 & 8 & & 13641.47 & & \\
\hline 7327.87 & 500 & $2 Z$ & 13642.77 & $28184_{4}^{\circ}-418273$ & -2 \\
\hline 7323.32 & 4 & & 13651.25 & & \\
\hline 7313.05 & 3000 & $50 Z$ & 13670.42 & $24751_{2}-38422_{1}^{\circ}$ & +1 \\
\hline 7310.70 & 400 & 1 & 13674.81 & $28857_{1}^{\circ}-42531_{2}$ & -4 \\
\hline 7305.23 & 2500 & $50 Z$ & 13685.05 & $24489_{1}-38174_{1}^{\circ}$ & -2 \\
\hline 7298.05 & $8 h$ & & 13698.52 & & \\
\hline 7256.62 & 20 & & 13776.72 & & \\
\hline 7253.76 & 40 & & 13782.16 & & \\
\hline 7244.41 & 2000 & $40 Z$ & 13799.94 & $24751_{2}-38551_{2}^{\circ}$ & -4 \\
\hline 7227.21 & 5 & & 13832.79 & & \\
\hline 7205.93 & 4 & & 13873.64 & & \\
\hline
\end{tabular}


TABLE 4. Observed and classified lines of $\mathrm{Yb} \mathrm{I}-$ Continued

\begin{tabular}{|c|c|c|c|c|c|}
\hline \multirow{2}{*}{$\begin{array}{l}\text { Wavelength } \\
\text { (air) } \\
\AA\end{array}$} & \multicolumn{2}{|c|}{ Intensity } & \multirow{2}{*}{$\underset{\mathrm{cm}^{-1}}{\text { Wavenumber }}$} & \multirow[b]{2}{*}{ Classification } & \multirow{2}{*}{$\begin{array}{c}\mathrm{O}-\mathrm{C} \\
0.01 \mathrm{~cm}^{-1}\end{array}$} \\
\hline & $\begin{array}{l}\text { Meggers } \\
\text { Lamp }\end{array}$ & $\begin{array}{c}\text { Thomson } \\
\text { Lamp }\end{array}$ & & & \\
\hline 7202.20 & 100 & 1 & 13880.82 & $488066-62687_{6}^{\circ}$ & -1 \\
\hline 7198.08 & $10 h$ & & 13888.77 & & \\
\hline 7196.92 & 3 & & 13891.00 & $32065_{3}-45956_{2}^{\circ}$ & +2 \\
\hline 7190.33 & 6 & & 13903.73 & & \\
\hline 7187.07 & 200 & $2 Z$ & 13910.04 & & \\
\hline 7175.10 & 1000 & 10 & 13933.25 & $244891-38422_{1}^{\circ}$ & -1 \\
\hline 7173.96 & 10 & & 13935.46 & & \\
\hline 7169.25 & 10 & & 13944.62 & & \\
\hline 7160.31 & 2 & & 13962.03 & & \\
\hline 7156.48 & 3 & & 13969.50 & & \\
\hline 7153.51 & 3 & & 13975.30 & & \\
\hline 7152.40 & 10 & & 13977.47 & $322734-46251_{3}^{\circ}$ & +1 \\
\hline 7141.28 & 6 & & 13999.23 & $486886-62687_{6}^{\circ}$ & +1 \\
\hline 7136.82 & 5 & & 14007.98 & & \\
\hline 7135.61 & 10 & & 14010.36 & & \\
\hline 7134.85 & 5 & & 14011.85 & & \\
\hline 7108.98 & 200 & 2 & 14062.84 & $24489_{1}-38551_{2}^{\circ}$ & +1 \\
\hline 7032.91 & 2 & & 14214.94 & & \\
\hline 7031.21 & 3 & & 14218.38 & & \\
\hline 7020.18 & 500 & 4 & 14240.72 & $28195_{2}^{\circ}-424361$ & -2 \\
\hline 7001.79 & 3 & & 14278.12 & $45775_{4}-60053 \stackrel{\circ}{\circ}$ & +2 \\
\hline 6990.51 & 100 & & 14301.16 & & \\
\hline 6976.89 & $3 h$ & & 14329.08 & & \\
\hline 6973.58 & 10 & & 14335.88 & $28195_{2}^{\circ}-42531_{2}$ & -3 \\
\hline 6965.46 & 1 & & 14352.59 & & \\
\hline 6958.11 & 300 & 3 & 14367.76 & $28857_{1}^{\circ}-43224_{2}$ & -1 \\
\hline 6953.29 & 1 & & 14377.71 & & \\
\hline 6951.38 & 50 & & 14381.67 & $27445_{3}^{\circ}-41827_{3}$ & +0 \\
\hline 6943.98 & 4 & & 14396.99 & $47911_{5}-62308^{\circ}$ & +1 \\
\hline 6914.84 & 2 & & 14457.66 & $29774_{4}^{\circ}-442324$ & -4 \\
\hline 6913.73 & 50 & 1 & 14459.98 & $305245-449844$ & -5 \\
\hline 6902.28 & 2 & & 14483.97 & $479115-62395_{5}^{\circ}$ & -6 \\
\hline 6892.61 & 5 & & 14504.29 & & \\
\hline 6891.94 & 20 & & 14505.70 & $30207_{3}^{\circ}-44713_{3}$ & -4 \\
\hline 6869.56 & 200 & 1 & 14552.96 & $30207_{3}^{\circ}-44760_{2}$ & -3 \\
\hline 6862.08 & $2 h$ & & 14568.82 & $491034-63672 \circ$ & -2 \\
\hline 6850.64 & 4 & & 14593.15 & $490795-63672_{5}^{\circ}$ & +1 \\
\hline 6833.56 & 4 & & 14629.62 & $48057_{5}-62687_{6}^{\circ}$ & -1 \\
\hline 6831.17 & 7 & & 14634.74 & $476734-623085$ & -1 \\
\hline 6817.16 & 100 & & 14664.82 & & \\
\hline 6813.65 & $5 h$ & & 14672.37 & $476365-623085$ & +2 \\
\hline 6806.67 & $10 h$ & & 14687.42 & & \\
\hline 6799.60 & $60000 R$ & $6000 Z$ & 14702.69 & $17992_{1}^{\circ}-326941$ & +1 \\
\hline 6782.17 & 1000 & $20 Z$ & 14740.47 & $25068_{1}^{\circ}-39808_{1}$ & -2 \\
\hline 6779.74 & 2 & & 14745.76 & & \\
\hline
\end{tabular}


TABLE 4. Observed and classified lines of $\mathrm{Yb} \mathrm{I}-$ Continued

\begin{tabular}{|c|c|c|c|c|c|}
\hline \multirow{2}{*}{$\begin{array}{c}\text { Wavelength } \\
\text { (air) } \\
\AA\end{array}$} & \multicolumn{2}{|c|}{ Intensity } & \multirow{2}{*}{$\underset{\substack{\text { Wavenumber } \\
\mathrm{cm}^{-1}}}{ }$} & \multirow{2}{*}{ Classification } & \multirow{2}{*}{$\begin{array}{c}\mathrm{O}-\mathrm{C} \\
0.01 \mathrm{~cm}^{-1}\end{array}$} \\
\hline & $\begin{array}{c}\text { Meggers } \\
\text { Lamp }\end{array}$ & $\begin{array}{l}\text { Thomson } \\
\text {. Lamp }\end{array}$ & & & \\
\hline 6777.22 & 600 & 10 & 14751.24 & $28184_{4}^{\circ}-42935_{5}$ & -3 \\
\hline 6773.47 & 2 & & 14759.41 & $476365-62395_{5}^{\circ}$ & +1 \\
\hline 6772.26 & 20 & & 14762.05 & & \\
\hline 6768.70 & 6000 & $1000 Z$ & 14769.81 & $25068_{1}^{\circ}-39838_{2}$ & +0 \\
\hline 6765.67 & 40 & 1 & 14776.42 & $326941-47471_{2}^{\circ}$ & +2 \\
\hline 6765.24 & 200 & $1 Z$ & 14777.36 & $30207_{3}^{\circ}-44984_{4}$ & -1 \\
\hline 6761.64 & $3 h$ & & 14785.23 & & \\
\hline 6757.29 & 1 & & 14794.75 & & \\
\hline 6753.01 & 2 & & 14804.12 & $326941-474981$ & -1 \\
\hline 6749.40 & 1000 & $20 Z$ & 14812.04 & $25068_{1}^{\circ}-39880_{2}$ & +1 \\
\hline 6732.17 & 2 & & 14849.95 & & \\
\hline 6731.02 & $5 h$ & & 14852.49 & & \\
\hline 6724.92 & 1 & & 14865.96 & $488066-63672 \circ$ & +5 \\
\hline 6720.85 & 2 & & 14874.96 & & \\
\hline 6719.87 & $6 h$ & & 14877.13 & & \\
\hline 6717.05 & 30 & & 14883.38 & & \\
\hline 6715.79 & 1000 & $20 Z$ & 14886.17 & $30524_{5}^{\circ}-45410_{5}$ & -2 \\
\hline 6707.91 & 3 & & 14903.66 & & \\
\hline 6707.76 & 5 & & 14903.99 & & \\
\hline 6702.48 & 2 & & 14915.73 & & \\
\hline 6701.26 & 15 & & 14918.45 & & \\
\hline 6697.64 & $10 h$ & & 14926.51 & & \\
\hline 6692.42 & 700 & $5 Z$ & 14938.15 & $29774_{4}^{\circ}-447133$ & -1 \\
\hline 6687.82 & 60 & & 14948.43 & $28857_{1}^{\circ}-43805_{1}$ & +2 \\
\hline 6678.17 & 20000 & $1000 Z$ & 14970.03 & $27677_{2}-42647_{2}^{\circ}$ & -3 \\
\hline 6667.82 & 50000 & $2000 Z$ & 14993.27 & $25068_{1}^{\circ}-40061_{2}$ & -2 \\
\hline 6656.70 & 2 & & 15018.31 & & \\
\hline 6643.55 & 10000 & $300 Z$ & 15048.04 & $27677_{2}-42725_{2}^{\circ}$ & -6 \\
\hline 6642.12 & $10 h$ & & 15051.28 & $\left\{\begin{array}{l}476365-62687_{6}^{\circ} \\
49127_{1}^{\circ}-64178_{2}\end{array}\right.$ & $\left\{\begin{array}{l}-1 \\
-4\end{array}\right.$ \\
\hline 6640.79 & 7 & & 15054.29 & & \\
\hline 6639.34 & 150 & 1 & 15057.58 & & \\
\hline 6636.42 & $6 h$ & & 15064.20 & & \\
\hline 6634.28 & 5 & & 15069.06 & $449844-60053 \stackrel{\circ}{\circ}$ & +3 \\
\hline 6630.85 & $2 h$ & & 15076.86 & & \\
\hline 6626.73 & 800 & $2 Z$ & 15086.23 & $27445_{3}^{\circ}-42531_{2}$ & +0 \\
\hline 6625.27 & $4 h$ & & 15089.56 & & \\
\hline 6607.07 & 2000 & $40 Z$ & 15131.12 & $30207_{3}^{\circ}-45338_{2}$ & -3 \\
\hline 6594.65 & $3 h$ & & 15159.62 & & \\
\hline 6592.47 & 40 & & 15164.63 & $32694_{1}-47859_{1}^{\circ}$ & +2 \\
\hline 6572.90 & 300 & 2 & 15209.78 & $29774_{4}^{\circ}-44984_{4}$ & -1 \\
\hline 6568.35 & 60 & 1 & 15220.32 & $470885-623085$ & +2 \\
\hline 6565.72 & 1 & & 15226.42 & & \\
\hline 6561.65 & 3 & & 15235.86 & & \\
\hline 6555.15 & 1500 & $15 Z$ & 15250.97 & $30524_{5}^{\circ}-45775_{4}$ & +0 \\
\hline
\end{tabular}


TABLE 4. Observed and classified lines of $\mathrm{Yb} \mathrm{I}-$ Continued

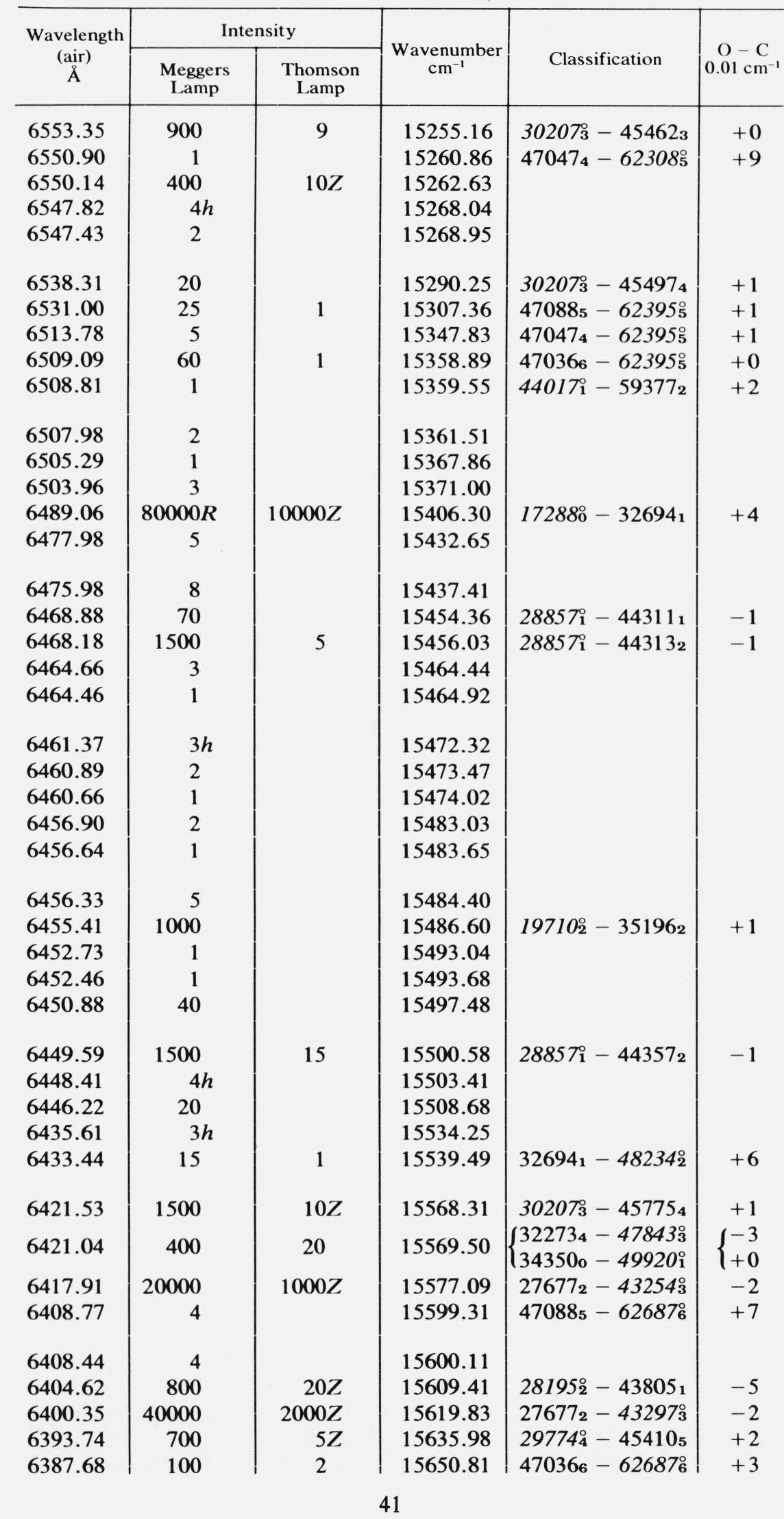


TABLE 4. Observed and classified lines of Yb I-Continued

\begin{tabular}{|c|c|c|c|c|c|}
\hline \multirow{2}{*}{$\begin{array}{l}\text { Wavelength } \\
\text { (air) } \\
\AA\end{array}$} & \multicolumn{2}{|c|}{ Intensity } & \multirow{2}{*}{$\underset{\mathrm{cm}^{-1}}{\text { Wavenumber }}$} & \multirow[b]{2}{*}{ Classification } & \multirow{2}{*}{$\begin{array}{c}\mathrm{O}-\mathrm{C} \\
0.01 \mathrm{~cm}^{-1}\end{array}$} \\
\hline & $\begin{array}{c}\text { Meggers } \\
\text { Lamp }\end{array}$ & $\begin{array}{l}\text { Thomson } \\
\text { Lamp }\end{array}$ & & & \\
\hline 6381.68 & 10 & & 15665.52 & $322734-47939_{4}^{\circ}$ & +2 \\
\hline 6372.71 & 1500 & $20 Z$ & 15687.57 & $29774_{4}^{\circ}-454623$ & -1 \\
\hline 6365.05 & 30 & & 15706.45 & $30207_{3}^{\circ}-45913_{2}$ & -3 \\
\hline 6359.22 & $2 h$ & & 15720.85 & & \\
\hline 6358.49 & 20 & & 15722.66 & $29774_{4}^{\circ}-454974$ & +0 \\
\hline 6348.85 & $2 h$ & & 15746.53 & & \\
\hline 6344.97 & 3000 & $100 Z$ & 15756.16 & $27677_{2}-43433_{2}^{\circ}$ & -3 \\
\hline 6343.01 & 8 & & 15761.03 & $479115-63672 \circ$ & +3 \\
\hline 6336.23 & 100 & 1 & 15777.89 & $32065_{3}-47843_{3}^{\circ}$ & +5 \\
\hline 6335.72 & 3000 & $70 Z$ & 15779.16 & $27445_{3}^{\circ}-43224_{2}$ & +2 \\
\hline 6328.19 & 4 & & 15797.94 & & \\
\hline 6322.56 & 400 & 3 & 15812.01 & $24751_{2}-40563_{1}^{\circ}$ & -2 \\
\hline 6318.91 & 20 & 1 & 15821.14 & $442324-600535$ & +2 \\
\hline 6311.10 & 2 & & 15840.72 & $465546-623955$ & +2 \\
\hline 6309.82 & 20 & 1 & 15843.93 & & \\
\hline 6305.37 & 90 & 1 & 15855.11 & $27677_{2}-43532_{1}^{\circ}$ & +1 \\
\hline 6304.79 & 8 & & 15856.57 & & \\
\hline 6299.71 & 2 & & 15869.36 & & \\
\hline 6297.95 & 90 & 1 & 15873.79 & $32065_{3}-47939_{4}^{\circ}$ & -3 \\
\hline 6286.25 & 2000 & $20 Z$ & 15903.34 & $28857_{1}^{\circ}-44760_{2}$ & -2 \\
\hline 6262.43 & 1 & & 15963.83 & $32273_{4}-48237_{3}^{\circ}$ & +3 \\
\hline 6261.50 & 2 & & 15966.20 & & \\
\hline 6257.03 & 20 & & 15977.60 & $28857_{1}^{\circ}-44834_{1}$ & +1 \\
\hline 6255.43 & 200 & 2 & 15981.69 & $27677_{2}-43659_{1}^{\circ}$ & -2 \\
\hline 6247.99 & 1500 & $15 Z$ & 16000.72 & $29774_{4}^{\circ}-45775_{4}$ & +0 \\
\hline 6244.69 & 3 & & 16009.18 & & \\
\hline 6236.55 & 600 & 5 & 16030.07 & $30524 \stackrel{\circ}{-}-465546$ & -2 \\
\hline 6235.25 & 200 & 3 & 16033.41 & & \\
\hline 6234.12 & 25 & & 16036.32 & $476365-63672 \stackrel{\circ}{5}$ & -5 \\
\hline 6228.53 & 1 & & 16050.71 & & \\
\hline 6219.16 & 15 & & 16074.89 & $244891-40563_{1}^{\circ}$ & +3 \\
\hline 6210.59 & 900 & 1 & 16097.08 & $19710_{2}^{\circ}-35807_{3}$ & -6 \\
\hline 6209.04 & 2 & & 16101.09 & & \\
\hline 6198.67 & 20 & & 16128.03 & $27677_{2}-43805_{2}^{\circ}$ & +1 \\
\hline 6196.90 & 3 & & 16132.64 & $465546-62687_{6}^{\circ}$ & +5 \\
\hline 6194.84 & 900 & $4 Z$ & 16138.00 & $27677_{2}-43815_{3}^{\circ}$ & +0 \\
\hline 6192.32 & 60 & 1 & 16144.57 & & \\
\hline 6183.01 & 2 & & 16168.88 & $32065_{3}-48234_{2}^{\circ}$ & +4 \\
\hline 6182.14 & 1 & & 16171.15 & & \\
\hline 6181.78 & 100 & $2 Z$ & 16172.10 & $32065_{3}-48237_{3}^{\circ}$ & -1 \\
\hline 6176.91 & 1 & & 16184.85 & $28195_{2}^{\circ}-44380_{3}$ & -1 \\
\hline 6175.62 & 150 & 50 & 16188.23 & $30207_{3}^{\circ}-46395_{3}$ & +1 \\
\hline 6172.53 & 5 & & 16196.33 & $28184_{4}^{\circ}-44380_{3}$ & +2 \\
\hline 6171.87 & 20 & & 16198.06 & & \\
\hline 6161.97 & 50 & & 16224.09 & $30207_{3}^{\circ}-46431_{2}$ & -2 \\
\hline
\end{tabular}


TABLE 4. Observed and classified lines of $\mathrm{Yb} \mathrm{I}-\mathrm{Continued}$

\begin{tabular}{|c|c|c|c|c|c|}
\hline \multirow{2}{*}{$\begin{array}{l}\text { Wavelength } \\
\text { (air) } \\
\AA\end{array}$} & \multicolumn{2}{|c|}{ Intensity } & \multirow{2}{*}{$\underset{\mathrm{cm}^{-1}}{\text { Wavenumber }}$} & \multirow[b]{2}{*}{ Classification } & \multirow{2}{*}{$\begin{array}{c}\mathrm{O}-\mathrm{C} \\
0.01 \mathrm{~cm}^{-1}\end{array}$} \\
\hline & $\begin{array}{l}\text { Meggers } \\
\text { Lamp }\end{array}$ & $\begin{array}{c}\text { Thomson } \\
\text { Lamp }\end{array}$ & & & \\
\hline 6160.74 & 6 & & 16227.32 & & \\
\hline 6156.02 & 2 & & 16239.77 & $438146-600535$ & +10 \\
\hline 6148.50 & 15 & & 16259.63 & $32065_{3}-48324_{2}^{\circ}$ & +2 \\
\hline 6148.24 & 1 & & 16260.32 & $30207_{3}^{\circ}-46467_{2}$ & +0 \\
\hline 6144.20 & $3 h$ & & 16271.01 & & \\
\hline 6130.67 & 1 & & 16306.92 & & \\
\hline 6118.28 & 3000 & $100 Z$ & 16339.94 & $27677_{2}-44017_{1}^{\circ}$ & +1 \\
\hline 6113.42 & 10 & & 16352.93 & & \\
\hline 6111.27 & 4000 & $60 Z$ & 16358.68 & $17992_{1}^{\circ}-34350 \mathrm{o}$ & +4 \\
\hline 6101.83 & 40 & & 16383.99 & $32065_{3}-48449_{2}^{\circ}$ & +0 \\
\hline 6096.15 & 15 & 2 & 16399.26 & & \\
\hline 6094.49 & 4 & & 16403.72 & & \\
\hline 6087.44 & 20 & & 16422.72 & $32065_{3}-48487_{3}^{\circ}$ & +2 \\
\hline 6086.48 & 2 & & 16425.31 & & \\
\hline 6073.17 & 7 & & 16461.31 & & \\
\hline 6066.51 & 15 & & 16479.38 & $36060_{4}-52540_{4}^{\circ}$ & +1 \\
\hline 6065.72 & 300 & $3 Z$ & 16481.53 & $28857_{1}^{\circ}-45338_{2}$ & +1 \\
\hline 6059.22 & 6000 & 80 & 16499.21 & $27314_{6}^{\circ}-438146$ & +2 \\
\hline 6054.57 & 4000 & $50 Z$ & 16511.88 & $30524^{\circ}-470366$ & -3 \\
\hline 6052.63 & 150 & & 16517.17 & $28195_{2}^{\circ}-447133$ & +1 \\
\hline 6050.48 & 2 & & 16523.04 & $30524^{\circ}-470474$ & +6 \\
\hline 6048.44 & 4000 & $50 Z$ & 16528.61 & $28184_{4}^{\circ}-44713_{3}$ & +0 \\
\hline 6046.91 & 9 & & 16532.79 & $45775_{4}-623085$ & +1 \\
\hline 6045.95 & $6 h$ & & 16535.42 & & \\
\hline 6044.96 & $3 h$ & & 16538.13 & & \\
\hline 6041.79 & 100 & 1 & 16546.80 & $25068_{1}^{\circ}-416151$ & -1 \\
\hline 6035.72 & 400 & $4 Z$ & 16563.44 & $30524_{5}^{\circ}-47088_{5}$ & +0 \\
\hline 6035.37 & 3 & & 16564.41 & $28195_{2}^{\circ}-44760_{2}$ & +0 \\
\hline 6034.26 & 1 & & 16567.45 & & \\
\hline 6031.80 & 3000 & $40 Z$ & 16574.21 & $27677 \mathbf{2}-44251_{2}^{\circ}$ & -1 \\
\hline 6029.05 & $7 h$ & & 16581.77 & & \\
\hline 6027.50 & $1 h$ & & 16586.03 & & \\
\hline 6023.82 & $2 h l$ & & 16596.17 & & \\
\hline 6018.16 & $5 h$ & & 16611.77 & & \\
\hline 6014.95 & 1500 & $10 Z$ & 16620.64 & $29774_{4}^{\circ}-46395_{3}$ & +0 \\
\hline 6014.16 & 10 & & 16622.82 & & \\
\hline 6011.18 & 1 & & 16631.06 & & \\
\hline 6008.44 & 200 & 1 & 16638.65 & $28195_{2}^{\circ}-44834_{1}$ & +0 \\
\hline 6004.52 & 2000 & $20 Z$ & 16649.51 & $23188_{2}^{\circ}-39838_{2}$ & -1 \\
\hline 6003.62 & 400 & $10 Z$ & 16652.00 & & \\
\hline 6003.33 & $2 h l$ & & 16652.81 & & \\
\hline 6000.28 & 20 & & 16661.27 & & \\
\hline 5989.33 & 5000 & $100 Z$ & 16691.73 & $23188_{2}^{\circ}-39880_{2}$ & -1 \\
\hline 5987.58 & 20 & & 16696.61 & & \\
\hline 5984.98 & $3 h l$ & & 16703.87 & & \\
\hline
\end{tabular}


TABLE 4. Observed and classified lines of $\mathrm{Yb} \mathrm{I}-$ Continued

\begin{tabular}{|c|c|c|c|c|c|}
\hline \multirow{2}{*}{$\begin{array}{l}\text { Wavelength } \\
\text { (air) } \\
\AA\end{array}$} & \multicolumn{2}{|c|}{ Intensity } & \multirow{2}{*}{$\underset{\mathrm{cm}^{-1}}{\text { Wavenumber }}$} & \multirow[b]{2}{*}{ Classification } & \multirow{2}{*}{$\begin{array}{c}O-C \\
0.01 \mathrm{~cm}^{-1}\end{array}$} \\
\hline & $\begin{array}{l}\text { Meggers } \\
\text { Lamp }\end{array}$ & $\begin{array}{l}\text { Thomson } \\
\text { Lamp }\end{array}$ & & & \\
\hline 5983.12 & 2 & & 16709.06 & & \\
\hline 5980.83 & 2 & & 16715.46 & & \\
\hline 5978.35 & 2 & & 16722.39 & & \\
\hline 5976.21 & 20 & & 16728.38 & & \\
\hline 5975.70 & 2 & & 16729.81 & & \\
\hline 5975.29 & 2 & & 16730.96 & & \\
\hline 5974.63 & 2 & & 16732.80 & $35807_{3}-52540_{4}^{\circ}$ & -3 \\
\hline 5972.73 & 150 & 1 & 16738.13 & $28857_{1}^{\circ}-45595_{1}$ & +0 \\
\hline 5969.95 & 3 & & 16745.92 & & \\
\hline 5969.84 & 2 & & 16746.23 & & \\
\hline 5968.23 & $2 h l$ & & 16750.75 & & \\
\hline 5966.86 & 2 & & 16754.59 & & \\
\hline 5966.43 & 20 & & 16755.80 & & \\
\hline 5965.48 & 1 & & 16758.47 & & \\
\hline 5963.60 & 2 & & 16763.75 & & \\
\hline 5960.20 & 1 & & 16773.31 & & \\
\hline 5960.04 & 10 & & 16773.76 & & \\
\hline 5959.33 & 1000 & $10 Z$ & 16775.76 & $27677_{2}-44453_{3}^{\circ}$ & -4 \\
\hline 5958.70 & 4000 & $100 Z$ & 16777.54 & $23188_{2}^{\circ}-399663$ & -4 \\
\hline 5955.34 & 1500 & $8 Z$ & 16787.00 & $27445_{3}^{\circ}-442324$ & -2 \\
\hline 5954.19 & $2 h$ & & 16790.24 & & \\
\hline 5950.66 & 1500 & $10 Z$ & 16800.20 & $28184_{4}^{\circ}-449844$ & -3 \\
\hline 5947.71 & 2 & & 16808.54 & & \\
\hline 5947.54 & 1 & & 16809.02 & & \\
\hline 5946.79 & $4 h l$ & & 16811.14 & & \\
\hline 5946.23 & 1 & & 16812.72 & & \\
\hline 5945.44 & 8 & & 16814.95 & & \\
\hline 5945.33 & 3 & & 16815.26 & & \\
\hline 5945.27 & 3 & & 16815.44 & & \\
\hline 5945.10 & 4 & & 16815.92 & & \\
\hline 5944.93 & 2 & & 16816.40 & & \\
\hline 5944.73 & 4 & & 16816.96 & & \\
\hline 5944.41 & 3 & & 16817.87 & & \\
\hline 5944.24 & 2 & & 16818.35 & & \\
\hline 5943.63 & 9 & & 16820.07 & & \\
\hline 5942.89 & 3 & & 16822.17 & & \\
\hline 5942.73 & 3 & & 16822.62 & & \\
\hline 5942.02 & 4 & & 16824.63 & & \\
\hline 5941.72 & 2 & & 16825.48 & & \\
\hline 5941.04 & 1 & & 16827.41 & & \\
\hline 5940.68 & 20 & & 16828.43 & & \\
\hline 5936.49 & 900 & $10 Z$ & 16840.30 & $30207_{3}^{\circ}-47047_{4}$ & +0 \\
\hline 5935.88 & 2 & & 16842.03 & & \\
\hline 5935.56 & 2 & & 16842.94 & & \\
\hline 5934.27 & 3 & & 16846.60 & & \\
\hline
\end{tabular}


TABLE 4. Observed and classified lines of $\mathrm{Yb} \mathrm{I}$-Continued

\begin{tabular}{|c|c|c|c|c|c|}
\hline \multirow{2}{*}{$\begin{array}{c}\text { Wavelength } \\
\text { (air) } \\
\AA\end{array}$} & \multicolumn{2}{|c|}{ Intensity } & \multirow{2}{*}{$\underset{\mathrm{cm}^{-1}}{\text { Wavenumber }}$} & \multirow[b]{2}{*}{ Classification } & \multirow{2}{*}{$\begin{array}{c}\mathrm{O}-\mathrm{C} \\
0.01 \mathrm{~cm}^{-1}\end{array}$} \\
\hline & $\begin{array}{l}\text { Meggers } \\
\text { Lamp }\end{array}$ & $\begin{array}{l}\text { Thomson } \\
\text { Lamp }\end{array}$ & & & \\
\hline 5933.775 & 2 & & 16848.01 & & \\
\hline 5932.98 & 2 & & 16850.27 & & \\
\hline 5932.562 & 4 & & 16851.46 & & \\
\hline 5931.853 & 3 & & 16853.47 & $47325_{3}^{\circ}-64178_{2}$ & -4 \\
\hline 5931.73 & $3 h$ & & 16853.82 & & \\
\hline 5930.722 & 6 & & 16856.68 & & \\
\hline 5929.82 & 4 & & 16859.25 & & \\
\hline 5928.761 & 5 & & 16862.26 & & \\
\hline 5928.63 & 2 & & 16862.63 & & \\
\hline 5927.69 & 60 & & 16865.30 & & \\
\hline 5926.958 & 10 & & 16867.39 & $27445_{3}^{\circ}-44313_{2}$ & -2 \\
\hline 5926.688 & 5 & & 16868.16 & & \\
\hline 5925.46 & 600 & $5 Z$ & 16871.65 & $25068_{1}^{\circ}-419390$ & -2 \\
\hline 5925.00 & 60 & & 16872.96 & $23188_{2}^{\circ}-40061_{2}$ & -3 \\
\hline 5924.498 & 15 & & 16874.39 & & \\
\hline 5924.165 & 2 & & 16875.34 & & \\
\hline 5922.991 & 5 & & 16878.69 & & \\
\hline 5922.153 & 15 & & 16881.07 & & \\
\hline 5920.48 & 2 & & 16885.84 & & \\
\hline 5919.710 & 8 & & 16888.04 & & \\
\hline 5919.585 & 2 & & 16888.40 & & \\
\hline 5917.826 & 3 & & 16893.42 & & \\
\hline 5917.130 & 6 & & 16895.40 & & \\
\hline 5916.00 & $5 h l$ & & 16898.63 & & \\
\hline 5915.052 & 3 & & 16901.34 & & \\
\hline 5914.415 & 5 & & 16903.16 & & \\
\hline 5912.35 & $5 h l$ & & 16909.06 & & \\
\hline 5912.15 & 2 & & 16909.64 & & \\
\hline 5911.55 & 5 & & 16911.35 & & \\
\hline 5911.35 & 20 & & 16911.92 & $27445^{\circ}-44357_{2}$ & -4 \\
\hline 5910.10 & 4 & & 16915.50 & & \\
\hline 5909.10 & 2 & & 16918.36 & & \\
\hline 5906.45 & 2 & & 16925.95 & & \\
\hline 5905.85 & 2 & & 16927.67 & & \\
\hline 5905.22 & 3 & & 16929.48 & & \\
\hline 5902.71 & 2 & & 16936.68 & & \\
\hline 5899.30 & 2 & & 16946.47 & & \\
\hline 5895.16 & 2 & & 16958.37 & & \\
\hline 5894.98 & 5 & & 16958.89 & & \\
\hline 5886.05 & 5 & & 16984.61 & $454105-623955$ & +2 \\
\hline 5877.68 & 10 & & 17008.80 & $32273_{4}-49282_{4}^{\circ}$ & +4 \\
\hline 5861.11 & 10 & & 17056.89 & $28857_{1}^{\circ}-45913_{2}$ & +4 \\
\hline 5854.510 & 4000 & $200 Z$ & 17076.12 & $258595-429355$ & +2 \\
\hline 5845.50 & $6 h$ & & 17102.44 & & \\
\hline 5842.44 & 80 & 1 & 17111.39 & $305245-476365$ & +0 \\
\hline
\end{tabular}


TABLE 4. Observed and classified lines of $\mathrm{Yb}$ I-Continued

\begin{tabular}{|c|c|c|c|c|c|}
\hline \multirow{2}{*}{$\begin{array}{c}\text { Wavelength } \\
\text { (air) } \\
\AA\end{array}$} & \multicolumn{2}{|c|}{ Intensity } & \multirow{2}{*}{$\underset{\substack{\text { Wavenumber } \\
\mathrm{cm}^{-1}}}{ }$} & \multirow[b]{2}{*}{ Classification } & \multirow{2}{*}{$\begin{array}{c}\mathrm{O}-\mathrm{C} \\
0.01 \mathrm{~cm}^{-1}\end{array}$} \\
\hline & $\begin{array}{l}\text { Meggers } \\
\text { Lamp }\end{array}$ & $\begin{array}{c}\text { Thomson } \\
\text { Lamp }\end{array}$ & & & \\
\hline 5841.96 & 20 & & 17112.80 & & \\
\hline 5839.25 & $2 h$ & & 17120.74 & & \\
\hline 5838.91 & $6 h$ & & 17121.74 & & \\
\hline 5838.27 & 70 & 1 & 17123.61 & $47055_{2}^{\circ}-64178_{2}$ & -4 \\
\hline 5834.58 & 200 & 3 & 17134.44 & $30207_{3}^{\circ}-47341_{2}$ & +0 \\
\hline 5833.62 & 20 & & 17137.26 & & \\
\hline 5831.82 & 300 & $3 Z$ & 17142.55 & $28195_{2}^{\circ}-45338_{2}$ & -2 \\
\hline 5830.82 & 2 & & 17145.49 & & \\
\hline 5829.63 & 200 & 2 & 17148.99 & $30524_{5}^{\circ}-476734$ & +0 \\
\hline 5829.11 & $5 h$ & & 17150.52 & & \\
\hline 5820.17 & 2 & & 17176.87 & & \\
\hline 5812.64 & $3 h$ & & 17199.12 & & \\
\hline 5810.67 & 3000 & $7 Z$ & 17204.95 & $17992_{1}^{\circ}-351962$ & -2 \\
\hline 5806.58 & 7 & & 17217.07 & $32065_{3}-49282^{\circ}$ & -1 \\
\hline 5804.06 & 80 & 1 & 17224.54 & $28857_{1}^{\circ}-46081_{0}$ & +2 \\
\hline 5803.44 & 5000 & $80 Z$ & 17226.38 & $28184_{4}^{\circ}-45410_{5}$ & -1 \\
\hline 5802.36 & 200 & 1 & 17229.59 & & \\
\hline 5798.82 & $2 h$ & & 17240.11 & & \\
\hline 5796.09 & 80 & & 17248.23 & $30524^{\circ}-477724$ & +0 \\
\hline 5789.93 & 1500 & 15 & 17266.58 & $28195_{2}^{\circ}-45462_{3}$ & +0 \\
\hline 5789.63 & 100 & & 17267.47 & $27445_{3}^{\circ}-447133$ & -1 \\
\hline 5787.86 & 500 & 5 & 17272.75 & $29774_{4}^{\circ}-470474$ & +2 \\
\hline 5786.09 & 400 & 3 & 17278.04 & $28184_{4}^{\circ}-454623$ & +1 \\
\hline 5784.43 & $3 h$ & & 17283.00 & & \\
\hline 5784.026 & 10 & & 17284.20 & & \\
\hline 5774.34 & 500 & 5 & 17313.19 & $\left\{\begin{array}{l}28184_{4}^{\circ}-45497_{4} \\
29774_{4}^{\circ}-47088_{5}\end{array}\right.$ & $\left\{\begin{array}{l}+9 \\
-1\end{array}\right.$ \\
\hline 5773.82 & 1000 & 10 & 17314.75 & $27445_{3}^{\circ}-44760_{2}$ & +2 \\
\hline 5772.39 & $3 h l$ & & 17319.04 & & \\
\hline 5765.21 & $5 h$ & & 17340.61 & & \\
\hline 5759.84 & $4 h$ & & 17356.78 & & \\
\hline 5758.25 & 9 & & 17361.57 & $351785-52540_{4}^{\circ}$ & +0 \\
\hline 5755.89 & 3000 & $40 Z$ & 17368.69 & $25068_{1}^{\circ}-424360$ & +1 \\
\hline 5753.20 & 2 & & 17376.81 & $25270_{3}-42647_{2}^{\circ}$ & -1 \\
\hline 5749.91 & 1000 & $400 Z$ & 17386.75 & $30524 \stackrel{\circ}{ }-479115$ & -1 \\
\hline 5745.80 & 2000 & $30 Z$ & 17399.19 & $28195_{2}^{\circ}-45595_{1}$ & +1 \\
\hline 5741.99 & 15 & & 17410.74 & $449844-62395_{5}^{\circ}$ & -2 \\
\hline 5738.53 & 2 & & 17421.23 & & \\
\hline 5733.00 & $2 h l$ & & 17438.04 & & \\
\hline 5732.60 & $4 h l$ & & 17439.25 & $30207_{3}^{\circ}-476463$ & +1 \\
\hline 5731.94 & $6 h l$ & & 17441.26 & & \\
\hline 5731.26 & $6 h l$ & & 17443.33 & & \\
\hline 5730.41 & $7 h l$ & & 17445.92 & & \\
\hline 5729.26 & $5 h l$ & & 17449.42 & & \\
\hline 5728.853 & 150 & $5 Z$ & 17450.66 & & \\
\hline
\end{tabular}


TABLE 4. Observed and classified lines of $\mathrm{Yb} \mathrm{I}-\mathrm{Continued}$

\begin{tabular}{|c|c|c|c|c|c|}
\hline \multirow{2}{*}{$\begin{array}{c}\text { Wavelength } \\
\text { (air) } \\
\AA\end{array}$} & \multicolumn{2}{|c|}{ Intensity } & \multirow{2}{*}{$\begin{array}{l}\text { Wavenumber } \\
\mathrm{cm}^{-1}\end{array}$} & \multirow[b]{2}{*}{ Classification } & \multirow{2}{*}{$\begin{array}{c}\mathrm{O}-\mathrm{C} \\
0.01 \mathrm{~cm}^{-1}\end{array}$} \\
\hline & $\begin{array}{c}\text { Meggers } \\
\text { Lamp }\end{array}$ & $\begin{array}{c}\text { Thomson } \\
\text { Lamp }\end{array}$ & & & \\
\hline 5728.58 & $5 h l$ & & 17451.49 & & \\
\hline 5727.47 & 90 & & 17454.87 & $25270_{3}-42725_{2}^{\circ}$ & +2 \\
\hline 5726.68 & $10 h$ & & 17457.28 & & \\
\hline 5724.58 & 500 & $2 Z$ & 17463.69 & $25068_{1}^{\circ}-42531_{2}$ & +4 \\
\hline 5723.70 & 600 & 7 & 17466.37 & $30207_{3}^{\circ}-476734$ & +4 \\
\hline 5719.99 & 20000 & $1000 Z$ & 17477.70 & $27677_{2}-45155_{2}^{\circ}$ & +3 \\
\hline 5711.38 & 1 & & 17504.05 & $27677_{2}-45181_{1}^{\circ}$ & +2 \\
\hline 5701.922 & 250 & $3 Z$ & 17533.08 & $30524 \stackrel{\circ}{5}-48057_{5}$ & +3 \\
\hline 5699.95 & 3000 & $50 Z$ & 17539.15 & $27445_{3}^{\circ}-44984_{4}$ & +4 \\
\hline 5698.21 & 2 & & 17544.50 & & \\
\hline 5696.93 & 2 & & 17548.45 & & \\
\hline 5689.917 & 1000 & $30 Z$ & 17570.07 & & \\
\hline 5688.48 & 200 & 3 & 17574.51 & $28857_{1}^{\circ}-46431_{2}$ & +4 \\
\hline 5684.13 & 2 & & 17587.96 & $28857_{1}^{\circ}-46444_{1}$ & +2 \\
\hline 5683.09 & 500 & $5 Z$ & 17591.18 & $28184_{4}^{\circ}-45775_{4}$ & +1 \\
\hline 5670.02 & 500 & 2 & 17631.73 & & \\
\hline 5663.21 & 200 & 2 & 17652.93 & $30207_{3}^{\circ}-47860_{3}$ & +3 \\
\hline 5642.446 & 40 & & 17717.89 & $28195_{2}^{\circ}-45913_{2}$ & -1 \\
\hline 5628.958 & 10 & & 17760.35 & & \\
\hline 5625.92 & 20 & & 17769.94 & & \\
\hline 5616.66 & $2 h$ & & 17799.23 & & \\
\hline 5614.68 & 1 & & 17805.51 & & \\
\hline 5614.116 & 3 & & 17807.30 & & \\
\hline 5613.39 & $5 h$ & & 17809.60 & & \\
\hline 5611.50 & 1 & & 17815.60 & & \\
\hline 5610.60 & 2 & & 17818.46 & & \\
\hline 5610.281 & 3 & & 17819.47 & & \\
\hline 5609.44 & 1 & & 17822.14 & & \\
\hline 5608.041 & 6 & & 17826.59 & & \\
\hline 5605.847 & 3 & & 17833.57 & & \\
\hline 5600.45 & 10 & & 17850.75 & & \\
\hline 5598.14 & 2 & & 17858.12 & & \\
\hline 5597.189 & 800 & $10 Z$ & 17861.15 & $29774_{4}^{\circ}-47636_{5}$ & +0 \\
\hline 5595.84 & 5 & & 17865.46 & & \\
\hline 5591.19 & 10 & & 17880.32 & & \\
\hline 5590.10 & 10 & & 17883.80 & & \\
\hline 5587.249 & 200 & 2 & 17892.93 & $27445_{3}^{\circ}-45338_{2}$ & +4 \\
\hline 5586.362 & 2000 & $100 Z$ & 17895.77 & $24751_{2}-42647_{2}^{\circ}$ & +0 \\
\hline 5585.426 & 400 & 5 & 17898.77 & $29774_{4}^{\circ}-47673_{4}$ & +2 \\
\hline 5578.232 & 400 & $20 Z$ & 17921.85 & & \\
\hline 5576.25 & 5 & & 17928.22 & $30207_{3}^{\circ}-48135_{2}$ & +0 \\
\hline 5568.11 & 1000 & $100 Z$ & 17954.43 & $258595-438146$ & +0 \\
\hline 5562.093 & 2000 & $200 Z$ & 17973.85 & $24751_{2}-42725_{2}^{\circ}$ & +4 \\
\hline 5558.98 & 800 & 8 & 17983.92 & $25270_{3}-43254_{3}^{\circ}$ & +4 \\
\hline 5556.466 & $50000 A$ & $10000 Z$ & 17992.05 & $0_{0}-17992_{1}^{\circ}$ & +5 \\
\hline
\end{tabular}


TABLE 4. Observed and classified lines of $\mathrm{Yb} \mathrm{I}-$ Continued

\begin{tabular}{|c|c|c|c|c|c|}
\hline \multirow{2}{*}{$\begin{array}{l}\text { Wavelength } \\
\text { (air) } \\
\AA\end{array}$} & \multicolumn{2}{|c|}{ Intensity } & \multirow{2}{*}{$\underset{\substack{\text { Wavenumber } \\
\mathrm{cm}^{-1}}}{ }$} & \multirow[b]{2}{*}{ Classification } & \multirow{2}{*}{$\begin{array}{r}\mathrm{O}-\mathrm{C} \\
0.01 \mathrm{~cm}\end{array}$} \\
\hline & $\begin{array}{c}\text { Meggers } \\
\text { Lamp }\end{array}$ & $\begin{array}{c}\text { Thomson } \\
\text { Lamp }\end{array}$ & & & \\
\hline 5554.62 & 500 & 5 & 17998.03 & $29774_{4}^{\circ}-47772_{4}$ & +5 \\
\hline 5548.79 & 100 & & 18016.94 & $27445_{3}^{\circ}-454623$ & +4 \\
\hline 5545.814 & 500 & 10 & 18026.61 & $25270_{3}-43297_{3}^{\circ}$ & +0 \\
\hline 5539.053 & 20000 & $1000 Z$ & 18048.61 & & \\
\hline 5538.016 & 50 & & 18051.99 & $27445_{3}^{\circ}-45497_{4}$ & +1 \\
\hline 5535.47 & 7 & & 18060.30 & & \\
\hline 5528.403 & 3 & & 18083.38 & & \\
\hline 5527.81 & 50 & & 18085.32 & $29774_{4}^{\circ}-47860_{3}$ & +0 \\
\hline 5526.37 & 5 & & 18090.03 & & \\
\hline 5524.544 & 3000 & $30 Z$ & 18096.01 & $27314_{6}^{\circ}-45410_{5}$ & +2 \\
\hline 5521.76 & 10 & & 18105.14 & & \\
\hline 5518.352 & 70 & 1 & 18116.32 & & \\
\hline 5514.79 & 1 & & 18128.02 & & \\
\hline 5512.47 & 20 & & 18135.65 & & \\
\hline 5512.19 & 20 & & 18136.57 & $29774_{4}^{\circ}-47911_{5}$ & +5 \\
\hline 5506.11 & 1500 & 5 & 18156.60 & $25068_{1}^{\circ}-432242$ & +5 \\
\hline 5505.49 & 10000 & $300 Z$ & 18158.64 & $24489_{1}-42647_{2}^{\circ}$ & +3 \\
\hline 5504.17 & 60 & & 18163.00 & $25270_{3}-43433_{2}^{\circ}$ & +5 \\
\hline 5504.026 & 300 & 3 & 18163.47 & $30524^{\circ}-48688_{6}$ & +1 \\
\hline 5498.75 & 5000 & $500 Z$ & 18180.90 & & \\
\hline 5493.088 & 700 & $5 Z$ & 18199.64 & $28195_{2}^{\circ}-46395_{3}$ & +0 \\
\hline 5491.285 & 6 & & 18205.62 & $496385-67844_{6}^{\circ}$ & +1 \\
\hline 5489.633 & 80 & & 18211.09 & $28184_{4}^{\circ}-46395_{3}$ & +1 \\
\hline 5481.925 & 20000 & $400 Z$ & 18236.70 & $24489_{1}-42725_{2}^{\circ}$ & +4 \\
\hline 5476.90 & 2 & & 18253.43 & & \\
\hline 5474.565 & 400 & 5 & 18261.22 & & \\
\hline 5474.037 & 1500 & $15 Z$ & 18262.98 & $30524^{\circ}-48787_{4}$ & -2 \\
\hline 5469.353 & 400 & 5 & 18278.62 & $27677_{2}-45956_{2}^{\circ}$ & +1 \\
\hline 5468.387 & 150 & 2 & 18281.85 & $30524^{\circ}-488066$ & -1 \\
\hline 5468.099 & 1 & & 18282.81 & $29774_{4}^{\circ}-48057_{5}$ & +0 \\
\hline 5463.24 & 4 & & 18299.07 & & \\
\hline 5454.007 & 2500 & $25 Z$ & 18330.05 & $27445_{3}^{\circ}-45775_{4}$ & +1 \\
\hline 5453.47 & 60 & 1 & 18331.85 & & \\
\hline 5441.259 & 200 & 1 & 18372.99 & $25859^{\circ}-442324$ & +2 \\
\hline 5438.01 & 2 & & 18383.97 & & \\
\hline 5432.89 & 400 & 6 & 1.29 & $27677_{2}-46078_{1}^{\circ}$ & +5 \\
\hline 5425.445 & 100 & 1 & 18426.55 & $23188_{2}^{\circ}-41615_{1}$ & +2 \\
\hline 5421.361 & 7 & & 18440.43 & $30207_{3}^{\circ}-48647_{3}$ & +3 \\
\hline 5413.195 & 15 & & 18468.24 & $27445_{3}^{\circ}-45913_{2}$ & +2 \\
\hline 5408.348 & 100 & 2 & 18484.80 & $28857_{1}^{\circ}-47341_{2}$ & -1 \\
\hline 5405.541 & 60 & 1 & 18494.39 & $438146-623085$ & +4 \\
\hline 5404.899 & 300 & 5 & 18496.59 & $27677_{2}-46174_{i}^{\circ}$ & +5 \\
\hline 5403.079 & 3000 & $100 Z$ & 18502.82 & $24751_{2}-43254_{3}^{\circ}$ & -1 \\
\hline 5402.00 & 150 & 2 & 18506.52 & $27677_{2}-46184_{2}^{\circ}$ & +3 \\
\hline 5393.757 & 2500 & $90 Z$ & 18534.80 & $25270_{3}-43805_{2}^{\circ}$ & +1 \\
\hline
\end{tabular}


TABLE 4. Observed and classified lines of $\mathrm{Yb} \mathrm{I}-$ Continued

\begin{tabular}{|c|c|c|c|c|c|}
\hline \multirow{2}{*}{$\begin{array}{c}\text { Wavelength } \\
\text { (air) } \\
\AA\end{array}$} & \multicolumn{2}{|c|}{ Intensity } & \multirow{2}{*}{$\begin{array}{c}\text { Wavenumber } \\
\mathrm{cm}^{-1}\end{array}$} & \multirow[b]{2}{*}{ Classification } & \multirow{2}{*}{$\mid \begin{array}{c}O-C \\
0.01 \mathrm{~cm}^{-1}\end{array}$} \\
\hline & $\begin{array}{c}\text { Meggers } \\
\text { Lamp }\end{array}$ & $\begin{array}{l}\text { Thomson } \\
\text { Lamp }\end{array}$ & & & \\
\hline 5390.845 & 2500 & $40 Z$ & 18544.81 & $25270_{3}-43815^{\circ}$ & +4 \\
\hline 5390.622 & 4000 & $150 Z$ & 18545.58 & $24751_{2}-43297^{\circ}$ & +2 \\
\hline 5387.99 & 90 & 8 & 18554.64 & $30524 \stackrel{\circ}{-}-490795$ & +1 \\
\hline 5385.29 & 200 & 2 & 18563.94 & $28857_{1}^{\circ}-47420_{2}$ & -1 \\
\hline 5382.55 & 2 & & 18573.39 & $27677_{2}-46251_{3}^{\circ}$ & +0 \\
\hline 5380.94 & 1 & & 18578.95 & $30524^{\circ}-491034$ & +2 \\
\hline 5380.54 & 500 & $7 Z$ & 18580.33 & $30207_{3}^{\circ}-48787_{4}$ & +0 \\
\hline 5380.24 & 70 & $2 Z$ & 18581.36 & $438146-62395_{5}^{\circ}$ & -4 \\
\hline 5379.15 & 6 & & 18585.13 & & \\
\hline 5363.66 & 10000 & $200 Z$ & 18638.80 & $23188^{\circ}-418273$ & +2 \\
\hline 5351.29 & 5000 & $300 Z$ & 18681.89 & $24751_{2}-43433_{2}^{\circ}$ & -1 \\
\hline 5348.208 & 300 & 3 & 18692.65 & $27677_{2}-46370_{1}^{\circ}$ & +2 \\
\hline 5341.10 & 2 & & 18717.53 & & \\
\hline 5338.31 & 60 & & 18727.31 & $28857_{1}^{\circ}-47584_{1}$ & -1 \\
\hline 5335.82 & 10 & & 18736.05 & $30524^{\circ}-49260_{4}$ & +1 \\
\hline 5335.49 & 50 & & 18737.21 & $25068_{1}^{\circ}-438051$ & +2 \\
\hline 5330.79 & 1 & & 18753.73 & & \\
\hline 5330.348 & 6 & & 18755.28 & & \\
\hline 5327.593 & 4 & & 18764.98 & $490795-67844_{6}^{\circ}$ & -2 \\
\hline 5323.10 & 300 & $3 Z$ & 18780.82 & $24751_{2}-43532{ }^{\circ}$ & +0 \\
\hline 5320.962 & 5 & & 18788.37 & $28857_{1}^{\circ}-47645_{1}$ & -2 \\
\hline 5320.324 & 6 & & 18790.62 & & \\
\hline 5299.852 & 2000 & $10 Z$ & 18863.20 & $28184_{4}^{\circ}-47047_{4}$ & +3 \\
\hline 5297.153 & 150 & 4 & 18872.81 & $29774_{4}^{\circ}-48647_{3}$ & -1 \\
\hline 5297.02 & 50 & 1 & 18873.29 & $438146-62687_{6}^{\circ}$ & +0 \\
\hline 5290.578 & 150 & 3 & 18896.27 & $30207_{3}^{\circ}-49103_{4}$ & +1 \\
\hline 5288.51 & 2000 & $15 Z$ & 18903.66 & $28184_{4}^{\circ}-47088_{5}$ & +1 \\
\hline 5287.45 & 3000 & $70 Z$ & 18907.45 & $24751_{2}-43659_{1}^{\circ}$ & +2 \\
\hline 5277.04 & 30000 & $3000 Z$ & 18944.75 & $24489_{1}-43433_{2}^{\circ}$ & +0 \\
\hline 5275.592 & 1000 & $10 Z$ & 18949.94 & $27445_{3}^{\circ}-46395_{3}$ & -2 \\
\hline 5271.473 & 400 & 20 & 18964.75 & $28857_{1}^{\circ}-47821_{2}$ & -1 \\
\hline 5266.964 & 15 & & 18980.99 & $25270_{3}-44251_{2}^{\circ}$ & +1 \\
\hline 5265.558 & 40 & & 18986.05 & & \\
\hline 5264.238 & 10 & & 18990.82 & & \\
\hline 5258.165 & 800 & 10 & 19012.75 & $29774^{\circ}-48787_{4}$ & +0 \\
\hline 5257.29 & 90 & & 19015.91 & $30207_{3}^{\circ}-49223_{3}$ & -6 \\
\hline 5255.61 & 2 & & 19021.99 & $27445_{3}^{\circ}-46467_{2}$ & -7 \\
\hline 5253.448 & 1 & & 19029.82 & & \\
\hline 5250.804 & 100 & 2 & 19039.40 & $30207_{3}^{\circ}-49246_{2}$ & -1 \\
\hline 5247.94 & 15 & & 19049.79 & & \\
\hline 5246.857 & 400 & 4 & 19053.73 & $24751_{2}-43805_{2}^{\circ}$ & -2 \\
\hline 5244.11 & 10000 & $250 Z$ & 19063.71 & $24751_{2}-43815_{3}^{\circ}$ & -2 \\
\hline 5230.292 & 60 & & 19114.07 & $305245-496385$ & +5 \\
\hline 5228.172 & 1000 & $30 Z$ & 19121.82 & $25270_{3}-44392_{4}^{\circ}$ & +2 \\
\hline 5227.271 & 4000 & $80 Z$ & 19125.12 & $\left\{\begin{array}{l}244891-43614^{\circ} \\
258595-449844\end{array}\right.$ & $\begin{array}{l}-5 \\
+5\end{array}$ \\
\hline
\end{tabular}


TABLE 4. Observed and classified lines of $\mathrm{Yb} \mathrm{I}-$ Continued

\begin{tabular}{|c|c|c|c|c|c|}
\hline \multirow{2}{*}{$\begin{array}{c}\text { Wavelength } \\
\text { (air) } \\
\AA\end{array}$} & \multicolumn{2}{|c|}{ Intensity } & \multirow{2}{*}{$\underset{\mathrm{cm}^{-1}}{\text { Wavenumber }}$} & \multirow[b]{2}{*}{ Classification } & \multirow{2}{*}{$\begin{array}{c}\mathrm{O}-\mathrm{C} \\
0.01 \mathrm{~cm}^{-1}\end{array}$} \\
\hline & $\begin{array}{c}\text { Meggers } \\
\text { Lamp }\end{array}$ & $\begin{array}{c}\text { Thomson } \\
\text { Lamp }\end{array}$ & & & \\
\hline 5224.53 & 1 & & 19135.15 & & \\
\hline 5221.606 & 50 & 1 & 19145.87 & $28195_{2}^{\circ}-47341_{2}$ & +1 \\
\hline 5218.792 & 1 & & 19156.19 & $486886-67844_{6}^{\circ}$ & +3 \\
\hline 5214.95 & 500 & $5 Z$ & 19170.30 & $24489_{1}-43659_{1}^{\circ}$ & +2 \\
\hline 5211.604 & 6000 & $400 Z$ & 19182.61 & $25270_{3}-44453_{3}^{\circ}$ & +4 \\
\hline 5200.10 & 4 & & 19225.05 & $28195_{2}^{\circ}-47420_{2}$ & +5 \\
\hline 5196.81 & 40 & & 19237.22 & $30207_{3}^{\circ}-49444_{3}$ & +8 \\
\hline 5196.085 & 5000 & $200 Z$ & 19239.90 & $27314_{6}^{\circ}-465546$ & +1 \\
\hline 5195.20 & 100 & 1 & 19243.18 & $250681-44311_{1}$ & +3 \\
\hline 5194.752 & 2000 & 80 & 19244.84 & $25068_{1}^{\circ}-44313_{2}$ & +2 \\
\hline 5193.850 & 2000 & $20 Z$ & 19248.18 & $23188_{2}^{\circ}-424361$ & +0 \\
\hline 5189.14 & 200 & 2 & 19265.65 & $24751_{2}-44017_{1}^{\circ}$ & +0 \\
\hline 5182.755 & 2000 & $50 Z$ & 19289.38 & $25068_{1}^{\circ}-44357_{2}$ & +1 \\
\hline 5178.715 & 90 & 20 & 19304.43 & $29774_{4}^{\circ}-49079_{5}$ & +5 \\
\hline 5175.45 & 250 & 2 & 19316.61 & $24489_{1}-43805_{2}^{\circ}$ & +2 \\
\hline 5172.214 & 50 & 1 & 19328.70 & $29774_{4}^{\circ}-49103_{4}$ & +2 \\
\hline 5168.29 & 90 & & 19343.37 & $23188_{2}^{\circ}-42531_{2}$ & +2 \\
\hline 5163.862 & 1 & & 19359.96 & & \\
\hline 5160.47 & 7 & & 19372.68 & $429355-62308_{5}^{\circ}$ & +0 \\
\hline 5156.285 & 100 & 2 & 19388.41 & $28195_{2}^{\circ}-47584_{1}$ & +3 \\
\hline 5148.75 & 1 & & 19416.78 & & \\
\hline 5140.38 & 120 & 2 & 19448.40 & $29774_{4}^{\circ}-49223_{3}$ & +0 \\
\hline 5140.11 & 20 & & 19449.42 & $28195_{2}^{\circ}-47645_{1}$ & -2 \\
\hline 5139.53 & 900 & $10 Z$ & 19451.61 & $28184_{4}^{\circ}-47636_{5}$ & +2 \\
\hline 5139.35 & 40 & & 19452.29 & $28857_{1}^{\circ}-48309_{1}$ & -10 \\
\hline 5137.389 & 6 & & 19459.72 & $42935_{5}-62395_{5}^{\circ}$ & -1 \\
\hline 5136.76 & 2 & & 19462.10 & $28184_{4}^{\circ}-47646_{3}$ & -1 \\
\hline 5132.71 & 90 & 1 & 19477.46 & $28195_{2}^{\circ}-47673_{3}$ & -3 \\
\hline 5130.523 & 100 & 2 & 19485.76 & $29774_{4}^{\circ}-49260_{4}$ & -3 \\
\hline 5130.095 & 7 & & 19487.39 & & \\
\hline 5129.688 & 200 & 2 & 19488.93 & $28184_{4}^{\circ}-47673_{3}$ & +0 \\
\hline 5127.64 & 2 & & 19496.72 & & \\
\hline 5126.80 & 2000 & $20 Z$ & 19499.91 & $24751_{2}-44251_{2}^{\circ}$ & -2 \\
\hline 5119.29 & 100 & 1 & 19528.52 & $244891-44017_{1}^{\circ}$ & +2 \\
\hline 5114.606 & 40 & 1 & 19546.40 & & \\
\hline 5113.34 & 700 & $10 Z$ & 19551.24 & $25859_{5}^{\circ}-45410_{5}$ & +1 \\
\hline 5111.89 & 2 & & 19556.79 & & \\
\hline 5106.95 & 1 & & 19575.70 & & \\
\hline 5105.75 & 600 & $30 h l$ & 19580.30 & & \\
\hline 5104.85 & 2000 & $30 Z$ & 19583.76 & & \\
\hline 5103.724 & 5 & 1 & 19588.08 & & \\
\hline 5103.644 & 5 & & 19588.38 & $28184_{4}^{\circ}-47772_{4}$ & -4 \\
\hline 5100.08 & 100 & 1 & 19602.07 & $27445_{3}^{\circ}-470474$ & +2 \\
\hline 5093.92 & 2 & & 19625.78 & $28195_{2}^{\circ}-47821_{2}$ & -4 \\
\hline
\end{tabular}


TABLE 4. Observed and classified lines of $\mathrm{Yb} \mathrm{I}-$ Continued

\begin{tabular}{|c|c|c|c|c|c|}
\hline \multirow{2}{*}{$\begin{array}{c}\text { Wavelength } \\
\text { (air) } \\
\AA\end{array}$} & \multicolumn{2}{|c|}{ Intensity } & \multirow{2}{*}{$\begin{array}{c}\text { Wavenumber } \\
\mathrm{cm}^{-1}\end{array}$} & \multirow{2}{*}{ Classification } & \multirow{2}{*}{$\mid \begin{array}{c}\mathrm{O}-\mathrm{C} \\
0.01 \mathrm{~cm}^{-1}\end{array}$} \\
\hline & $\begin{array}{c}\text { Meggers } \\
\text { Lamp }\end{array}$ & $\begin{array}{l}\text { Thomson } \\
\text { Lamp }\end{array}$ & & & \\
\hline 5090.77 & 80 & & 19637.92 & $25859^{\circ}-454974$ & -2 \\
\hline 5088.275 & 15 & & 19647.55 & $27677_{2}-47325_{3}^{\circ}$ & +2 \\
\hline 5087.89 & 4 & & 19649.04 & $27677_{2}-47326^{\circ}$ & +5 \\
\hline 5086.45 & 15 & & 19654.60 & $27677_{2}-47332 i$ & +0 \\
\hline 5083.938 & 90 & 1 & 19664.31 & $28195_{2}^{\circ}-47860_{3}$ & -1 \\
\hline 5082.590 & 250 & 9 & 19669.53 & $29774_{4}^{\circ}-49444_{3}$ & -4 \\
\hline 5080.981 & 500 & $9 Z$ & 19675.75 & $28184_{4}^{\circ}-47860_{3}$ & -1 \\
\hline 5076.744 & 5000 & $150 Z$ & 19692.17 & $25068_{1}^{\circ}-44760_{2}$ & +3 \\
\hline 5074.34 & 20000 & $2000 Z$ & 19701.50 & $24751_{2}-44453_{3}^{\circ}$ & -2 \\
\hline 5069.144 & 4000 & $150 Z$ & 19721.70 & $273146-470366$ & +0 \\
\hline 5067.800 & 1500 & $40 Z$ & 19726.93 & $28184_{4}^{\circ}-47911_{5}$ & -4 \\
\hline 5063.605 & 8 & & 19743.27 & $27677_{2}-47420_{1}^{\circ}$ & +1 \\
\hline 5060.722 & 2 & & 19754.52 & & \\
\hline 5058.613 & 900 & $5 Z$ & 19762.75 & $24489_{1}-44251_{2}^{\circ}$ & -2 \\
\hline 5055.933 & 15 & & 19773.23 & $27314_{6}^{\circ}-470885$ & -1 \\
\hline 5055.485 & 2 & & 19774.98 & & \\
\hline 5050.77 & 15 & & 19793.44 & $27677_{2}-47471_{2}^{\circ}$ & +1 \\
\hline 5043.708 & 150 & $4 Z$ & 19821.16 & $27677_{2}-47498^{\circ}$ & -1 \\
\hline 5034.262 & 5 & 1 & 19858.35 & $438146-636725$ & -2 \\
\hline 5032.899 & 2 & & 19863.72 & $29774_{4}^{\circ}-496385$ & -5 \\
\hline 5032.14 & 2 & & 19866.72 & & \\
\hline 5029.03 & 6 & & 19879.01 & & \\
\hline 5027.67 & 2000 & $80 Z$ & 19884.38 & $25270_{3}-45155_{2}^{\circ}$ & -4 \\
\hline 5021.860 & 400 & 4 & 19907.39 & & \\
\hline 5019.691 & 1500 & $15 Z$ & 19915.99 & $25859^{\circ}-45775_{4}$ & -1 \\
\hline 5004.79 & 15 & & 19975.29 & $27445_{3}^{\circ}-47420_{2}$ & -4 \\
\hline 5003.306 & 2 & & 19981.21 & $28857_{1}^{\circ}-48838_{1}$ & -10 \\
\hline 4992.786 & 2 & & 20023.31 & & \\
\hline 4992.102 & 2 & & 20026.05 & $28857_{1}^{\circ}-48883_{2}$ & -4 \\
\hline 4989.924 & 6 & & 20034.80 & & \\
\hline 4989.57 & 100 & 1 & 20036.22 & $23188_{2}^{\circ}-43224_{2}$ & -4 \\
\hline 4986.88 & 6 & 3 & 20047.02 & & \\
\hline 4985.36 & 2 & & 20053.14 & $25068_{1}^{\circ}-45121_{1}$ & -1 \\
\hline 4982.82 & 2 & & 20063.36 & & \\
\hline 4981.34 & 1 & & 20069.32 & & \\
\hline 4974.16 & 2000 & $100 Z$ & 20098.29 & $19710_{2}^{\circ}-398081$ & -4 \\
\hline 4972.42 & 10 & 2 & 20105.32 & & \\
\hline 4970.41 & 40 & 7 & 20113.45 & $28195_{2}^{\circ}-48309_{1}$ & +0 \\
\hline 4966.902 & $10000 R$ & $700 Z$ & 20127.66 & $19710_{2}^{\circ}-39838_{2}$ & +1 \\
\hline 4962.28 & 1 & & 20146.41 & & \\
\hline 4960.28 & 1 & & 20154.53 & & \\
\hline 4957.83 & 8 & & 20164.49 & $28195_{2}^{\circ}-48360_{1}$ & +2 \\
\hline 4956.512 & 500 & $9 Z$ & 20169.85 & $19710_{2}^{\circ}-39880_{2}$ & -2 \\
\hline 4953.72 & 1 & & 20181.22 & & \\
\hline 4951.88 & 2 & & 20188.72 & $27445_{3}^{\circ}-47634_{2}$ & -6 \\
\hline
\end{tabular}


TABLE 4. Observed and classified lines of $\mathrm{Yb} \mathrm{I}-\mathrm{Continued}$

\begin{tabular}{|c|c|c|c|c|c|}
\hline \multirow{2}{*}{$\begin{array}{l}\text { Wavelength } \\
\text { (air) } \\
\AA\end{array}$} & \multicolumn{2}{|c|}{ Intensity } & \multirow{2}{*}{$\underset{\mathrm{cm}^{-1}}{\text { Wavenumber }}$} & \multirow[b]{2}{*}{ Classification } & \multirow{2}{*}{$\begin{array}{c}\mathrm{O}-\mathrm{C} \\
0.01 \mathrm{~cm}^{-1}\end{array}$} \\
\hline & $\begin{array}{c}\text { Meggers } \\
\text { Lamp }\end{array}$ & $\begin{array}{c}\text { Thomson } \\
\text { Lamp }\end{array}$ & & & \\
\hline 4942.254 & 250 & 3 & 20228.04 & $27445_{3}^{\circ}-47673_{4}$ & -3 \\
\hline 4935.500 & $20000 R$ & $2000 Z$ & 20255.72 & $19710_{2}^{\circ}-399663$ & +2 \\
\hline 4932.81 & 5 & & 20266.76 & $32273_{4}-52540_{4}^{\circ}$ & +1 \\
\hline 4931.953 & 1000 & $40 Z$ & 20270.28 & $25068_{1}^{\circ}-45338_{2}$ & -2 \\
\hline 4931.16 & 5 & & 20273.54 & & \\
\hline 4919.595 & 100 & 2 & 20321.20 & $27314_{6}^{\circ}-476365$ & +1 \\
\hline 4918.118 & 150 & $2 Z$ & 20327.31 & $27445_{3}^{\circ}-47772_{4}$ & +0 \\
\hline 4912.365 & 3000 & $100 Z$ & 20351.11 & $19710_{2}^{\circ}-40061_{2}$ & -1 \\
\hline 4906.335 & 50 & & 20376.12 & $27445_{3}^{\circ}-47821_{2}$ & -2 \\
\hline 4904.93 & 1 & & 20381.96 & & \\
\hline 4899.78 & 500 & $5 Z$ & 20403.38 & $24751_{2}-45155_{2}^{\circ}$ & +0 \\
\hline 4897.08 & 5 & & 20414.63 & $27445_{3}^{\circ}-47860_{3}$ & -1 \\
\hline 4895.606 & 150 & 5 & 20420.78 & & \\
\hline 4894.596 & 3000 & 400 & 20424.99 & & \\
\hline 4894.296 & 50 & & 20426.24 & & \\
\hline 4893.465 & 500 & $5 Z$ & 20429.71 & $24751_{2}-45181_{1}^{\circ}$ & -3 \\
\hline 4891.992 & 200 & $4 Z$ & 20435.86 & & \\
\hline 4888.18 & 25 & 1 & 20451.80 & $28195_{2}^{\circ}-486473$ & -2 \\
\hline 4885.45 & 90 & 3 & 20463.23 & $28184_{4}^{\circ}-48647_{3}$ & -4 \\
\hline 4882.15 & 2 & & 20477.06 & $27677_{2}-48154_{3}^{\circ}$ & +2 \\
\hline 4870.288 & 30 & & 20526.93 & $25068_{1}^{\circ}-455951$ & +2 \\
\hline 4868.511 & 8 & & 20534.43 & $27677_{2}-48212_{1}^{\circ}$ & -1 \\
\hline 4863.295 & 40 & & 20556.45 & $27677_{2}-48234_{2}^{\circ}$ & -1 \\
\hline 4862.524 & 2 & & 20559.71 & $27677_{2}-48237_{3}^{\circ}$ & -2 \\
\hline 4857.542 & 50 & 1 & 20580.79 & $27677_{2}-48258_{1}^{\circ}$ & -1 \\
\hline 4856.50 & 1 & & 20585.21 & & \\
\hline 4855.922 & 15 & & 20587.66 & & \\
\hline 4854.92 & 1 & & 20591.91 & & \\
\hline 4853.826 & 500 & $7 Z$ & 20596.55 & $27314 \%-479115$ & -1 \\
\hline 4849.034 & 100 & 1 & 20616.90 & $23188_{2}^{\circ}-43805_{1}$ & +0 \\
\hline 4845.612 & 3 & & 20631.46 & & \\
\hline 4841.918 & 50 & 2 & 20647.20 & $27677_{2}-48324_{2}^{\circ}$ & -2 \\
\hline 4839.462 & 2 & & 20657.68 & & \\
\hline 4838.657 & 7 & & 20661.12 & & \\
\hline 4837.46 & 4000 & $100 Z$ & 20666.23 & $24489_{1}-45155_{2}^{\circ}$ & +0 \\
\hline 4832.989 & 200 & 5 & 20685.35 & $25270_{3}-45956_{2}^{\circ}$ & -2 \\
\hline 4831.912 & 400 & 20 & 20689.96 & $27445_{3}^{\circ}-48135_{2}$ & +0 \\
\hline 4831.30 & 600 & $10 Z$ & 20692.58 & $24489_{1}-45181_{1}^{\circ}$ & -1 \\
\hline 4830.704 & 500 & 10 & 20695.13 & $258595-465546$ & +1 \\
\hline 4828.29 & 3 & & 20705.48 & & \\
\hline 4825.655 & 1 & & 20716.79 & & \\
\hline 4824.28 & 1 & & 20722.69 & & \\
\hline 4819.59 & 20 & 1 & 20742.86 & $27314_{6}^{\circ}-480575$ & +1 \\
\hline 4816.43 & 5000 & $600 Z$ & 20756.47 & & \\
\hline 4812.918 & 400 & $40 Z$ & 20771.61 & $27677_{2}-48449_{2}^{\circ}$ & +1 \\
\hline
\end{tabular}


TABLE 4. Observed and classified lines of $\mathrm{Yb} \mathrm{I}-$ Continued

\begin{tabular}{|c|c|c|c|c|c|}
\hline \multirow{2}{*}{$\begin{array}{c}\text { Wavelength } \\
\text { (air) } \\
\AA\end{array}$} & \multicolumn{2}{|c|}{ Intensity } & \multirow{2}{*}{$\underset{\mathrm{cm}^{-1}}{\text { Wavenumber }}$} & \multirow{2}{*}{ Classification } & \multirow{2}{*}{$\begin{array}{c}\mathrm{O}-\mathrm{C} \\
0.01 \mathrm{~cm}^{-1}\end{array}$} \\
\hline & $\begin{array}{l}\text { Meggers } \\
\text { Lamp }\end{array}$ & $\begin{array}{l}\text { Thomson } \\
\text { Lamp }\end{array}$ & & & \\
\hline 4811.89 & 1 & & 20776.05 & & \\
\hline 4810.51 & 40 & 4 & 20782.01 & & \\
\hline 4804.571 & 3 & & 20807.70 & $470366-678446$ & -2 \\
\hline 4800.535 & 2 & & 20825.19 & $38551_{2}^{\circ}-59377_{2}$ & -1 \\
\hline 4795.829 & 80 & 1 & 20845.63 & $25068_{1}^{\circ}-45913_{2}$ & -1 \\
\hline 4794.96 & 1 & & 20849.40 & & \\
\hline 4794.85 & 1 & & 20849.88 & & \\
\hline 4794.57 & 1 & & 20851.10 & & \\
\hline 4784.54 & 70 & 9 & 20894.81 & $28184_{4}^{\circ}-49079_{5}$ & -2 \\
\hline 4781.867 & 10000 & $2000 Z$ & 20906.49 & & \\
\hline 4780.32 & 400 & $15 Z$ & 20913.25 & $25270_{3}-46184_{2}^{\circ}$ & +1 \\
\hline 4778.982 & 200 & $7 Z$ & 20919.11 & $28184_{4}^{\circ}-491034$ & -2 \\
\hline 4770.834 & 150 & 2 & 20954.84 & $38422_{1}^{\circ}-59377_{2}$ & +7 \\
\hline 4765.51 & 1 & & 20978.25 & & \\
\hline 4765.080 & 3 & & 20980.14 & $25270_{3}-46251_{3}^{\circ}$ & -2 \\
\hline 4762.587 & 300 & $5 Z$ & 20991.12 & & \\
\hline 4761.55 & 2 & & 20995.69 & & \\
\hline 4758.320 & 80 & $4 Z$ & 21009.95 & & \\
\hline 4757.559 & 40 & 1 & 21013.31 & $25068^{\circ}-46081_{0}$ & -1 \\
\hline 4755.15 & 4 & & 21023.95 & $27677_{2}-48701_{2}^{\circ}$ & +2 \\
\hline 4754.370 & 5 & & 21027.40 & $28195_{2}^{\circ}-492233$ & +1 \\
\hline 4751.789 & 400 & $10 Z$ & 21038.82 & $28184_{4}^{\circ}-49223_{3}$ & -2 \\
\hline 4751.22 & 3 & & 21041.34 & $27677_{2}-48719_{1}^{\circ}$ & -2 \\
\hline 4745.27 & 2 & & 21067.72 & & \\
\hline 4743.356 & 250 & $7 Z$ & 21076.22 & $28184_{4}^{\circ}-49260_{4}$ & -1 \\
\hline 4741.574 & 8 & & 21084.15 & $27677_{2}-48761_{1}^{\circ}$ & -3 \\
\hline 4739.330 & 1 & & 21094.13 & & \\
\hline 4736.89 & 1 & & 21104.99 & & \\
\hline 4736.525 & 15 & & 21106.62 & & \\
\hline 4732.505 & 18 & & 21124.55 & $23188_{2}^{\circ}-443132$ & +2 \\
\hline 4732.14 & 2 & & 21126.18 & & \\
\hline 4731.04 & 2 & & 21131.09 & & \\
\hline 4723.85 & 1 & & 21163.25 & & \\
\hline 4722.53 & 2 & & 21169.17 & $23188_{2}^{\circ}-44357_{2}$ & +9 \\
\hline 4722.148 & 10 & & 21170.88 & & \\
\hline 4720.79 & 2000 & $50 Z$ & 21176.97 & $258595-470366$ & +3 \\
\hline 4718.56 & 3000 & $500 Z$ & 21186.98 & & \\
\hline 4718.34 & 200 & 2 & 21187.97 & $25859_{5}^{\circ}-47047_{4}$ & -4 \\
\hline 4717.375 & 30 & & 21192.30 & $23188_{2}^{\circ}-44380_{3}$ & +0 \\
\hline 4715.16 & 6 & & 21202.26 & $27445_{3}^{\circ}-48647_{3}$ & +11 \\
\hline 4714.700 & 700 & 40 & 21204.32 & $24751_{2}-45956_{2}^{\circ}$ & +0 \\
\hline 4712.73 & 2 & & 21213.19 & & \\
\hline 4709.96 & 15 & 1 & 21225.66 & & \\
\hline 4709.330 & 40 & 1 & 21228.50 & $258595-47088_{5}$ & +3 \\
\hline 4706.81 & 2 & & 21239.87 & & \\
\hline
\end{tabular}


TABLE 4. Observed and classified lines of $\mathrm{Yb} \mathrm{I}-$ Continued

\begin{tabular}{|c|c|c|c|c|c|}
\hline \multirow{2}{*}{$\begin{array}{l}\text { Wavelength } \\
\text { (air) } \\
\AA\end{array}$} & \multicolumn{2}{|c|}{ Intensity } & \multirow{2}{*}{$\underset{\mathrm{cm}^{-1}}{\text { Wavenumber }}$} & \multirow{2}{*}{ Classification } & \multirow{2}{*}{$\begin{array}{c}\mathrm{O}-\mathrm{C} \\
0.01 \mathrm{~cm}^{-1}\end{array}$} \\
\hline & $\begin{array}{c}\text { Meggers } \\
\text { Lamp }\end{array}$ & $\begin{array}{c}\text { Thomson } \\
\text { Lamp }\end{array}$ & & & \\
\hline 4706.700 & 2 & & 21240.36 & & \\
\hline 4704.886 & 300 & 20 & 21248.55 & $28195_{2}^{\circ}-49444_{3}$ & -1 \\
\hline 4702.982 & 10 & & 21257.16 & & \\
\hline 4702.355 & 200 & 10 & 21259.99 & $28184_{4}^{\circ}-49444_{3}$ & -2 \\
\hline 4696.286 & 40 & 2 & 21287.46 & $27677_{2}-48965_{2}^{\circ}$ & -3 \\
\hline 4695.832 & 7 & & 21289.52 & $465546-67844_{6}^{\circ}$ & -1 \\
\hline 4695.70 & 2 & & 21290.12 & & \\
\hline 4693.06 & 1 & & 21302.10 & & \\
\hline 4689.069 & 15 & & 21320.23 & & \\
\hline 4687.593 & 1000 & $50 Z$ & 21326.94 & $24751_{2}-460781$ & -2 \\
\hline 4685.28 & 4 & & 21337.47 & & \\
\hline 4684.268 & 1500 & $80 Z$ & 21342.08 & $27445_{3}^{\circ}-48787_{4}$ & +1 \\
\hline 4681.666 & 10 & 1 & 21353.94 & & \\
\hline 4681.514 & 3 & & 21354.63 & & \\
\hline 4680.136 & 6 & 1 & 21360.92 & & \\
\hline 4679.625 & 15 & & 21363.25 & $250681-46431_{2}$ & -1 \\
\hline 4677.432 & 90 & 1 & 21373.27 & $27314 \stackrel{\circ}{-}-486886$ & +1 \\
\hline 4676.67 & 6 & & 21376.75 & $25068_{1}^{\circ}-46444_{1}$ & +2 \\
\hline 4675.20 & 1 & & 21383.47 & & \\
\hline 4673.62 & 1 & & 21390.70 & & \\
\hline 4672.125 & 1 & & 21397.55 & & \\
\hline 4671.701 & 30 & 1 & 21399.49 & $250681-464672$ & +2 \\
\hline 4669.510 & 2 & & 21409.53 & & \\
\hline 4666.735 & 200 & $10 Z$ & 21422.26 & $24751_{2}-46174_{1}^{\circ}$ & +0 \\
\hline 4664.565 & 30 & & 21432.23 & $24751_{2}-46184_{2}^{\circ}$ & +2 \\
\hline 4663.873 & 5 & & 21435.41 & & \\
\hline 4663.44 & 1 & & 21437.40 & $27445_{3}^{\circ}-488832$ & -8 \\
\hline 4661.878 & 5 & & 21444.58 & & \\
\hline 4660.057 & 4 & & 21452.96 & $42725_{2}^{\circ}-64178_{2}$ & +2 \\
\hline 4659.780 & 20 & & 21454.23 & $28184_{4}^{\circ}-496385$ & +2 \\
\hline 4658.87 & $4 d$ & & 21458.43 & & \\
\hline 4658.443 & 2 & 2 & 21460.39 & & \\
\hline 4656.971 & 3000 & $500 Z$ & 21467.17 & $24489_{1}-45956_{2}^{\circ}$ & +1 \\
\hline 4654.46 & 4 & & 21478.76 & & \\
\hline 4653.310 & 5 & & 21484.06 & & \\
\hline 4651.67 & 500 & $20 Z$ & 21491.64 & $273149-488066$ & -1 \\
\hline 4650.99 & 3 & & 21494.78 & & \\
\hline 4650.05 & 1500 & $100 Z$ & 21499.13 & $24751_{2}-46251_{3}^{\circ}$ & +1 \\
\hline 4646.91 & 7 & & 21513.65 & & \\
\hline 4644.54 & 3000 & $100 Z$ & 21524.63 & $23188_{2}^{\circ}-447133$ & +3 \\
\hline 4641.945 & 6 & 2 & 21536.66 & & \\
\hline 4638.81 & 3 & & 21551.22 & & \\
\hline 4637.341 & 3 & & 21558.04 & & \\
\hline 4634.38 & 4 & & 21571.82 & $23188_{2}^{\circ}-44760_{2}$ & -3 \\
\hline 4633.196 & 30 & 4 & 21577.33 & & \\
\hline
\end{tabular}


TABLE 4. Observed and classified lines of $\mathrm{Yb} \mathrm{I}$-Continued

\begin{tabular}{|c|c|c|c|c|c|}
\hline \multirow{2}{*}{$\begin{array}{c}\text { Wavelength } \\
\text { (air) } \\
\AA\end{array}$} & \multicolumn{2}{|c|}{ Intensity } & \multirow{2}{*}{$\underset{\mathrm{cm}^{-1}}{\text { Wavenumber }}$} & \multirow[b]{2}{*}{ Classification } & \multirow{2}{*}{$\begin{array}{c}\mathrm{O}-\mathrm{C} \\
0.01 \mathrm{~cm}^{-1}\end{array}$} \\
\hline & $\begin{array}{c}\text { Meggers } \\
\text { Lamp }\end{array}$ & $\begin{array}{l}\text { Thomson } \\
\text { Lamp }\end{array}$ & & & \\
\hline 4631.09 & 2 & & 21587.14 & & \\
\hline 4630.522 & 20 & & 21589.79 & $24489_{1}-46078_{1}^{\circ}$ & -2 \\
\hline 4629.833 & 200 & 3 & 21593.00 & $244891-46082^{\circ}$ & -6 \\
\hline 4628.861 & 2 & & 21597.54 & & \\
\hline 4628.43 & 2 & & 21599.55 & & \\
\hline 4627.556 & 4 & & 21603.63 & & \\
\hline 4627.23 & 5 & & 21605.15 & & \\
\hline 4626.054 & 2 & & 21610.64 & & \\
\hline 4625.47 & 2 & & 21613.37 & & \\
\hline 4624.41 & 1200 & $50 Z$ & 21618.33 & $24751_{2}-46370_{1}^{\circ}$ & -3 \\
\hline 4621.80 & 3 & & 21630.53 & & \\
\hline 4618.487 & 15 & & 21646.05 & $23188_{2}^{\circ}-44834_{1}$ & -4 \\
\hline 4617.900 & 5 & & 21648.80 & & \\
\hline 4617.46 & 1 & & 21650.86 & & \\
\hline 4615.947 & 300 & $15 Z$ & 21657.96 & $27445_{3}^{\circ}-491034$ & -4 \\
\hline 4615.427 & 3 & & 21660.40 & & \\
\hline 4610.172 & 600 & $30 Z$ & 21685.09 & $24489_{1}-46174_{1}^{\circ}$ & -2 \\
\hline 4608.061 & 3 & & 21695.02 & $24489_{1}-46184_{2}^{\circ}$ & -2 \\
\hline 4606.854 & 1 & & 21700.71 & & \\
\hline 4602.354 & 2 & & 21721.93 & & \\
\hline 4594.033 & 10 & & 21761.27 & & \\
\hline 4593.375 & 30 & 5 & 21764.39 & $27314_{6}^{\circ}-49079_{5}$ & -3 \\
\hline 4591.780 & 5 & & 21771.95 & & \\
\hline 4590.834 & 2000 & $200 Z$ & 21776.43 & $258595-476365$ & +1 \\
\hline 4590.578 & 10 & & 21777.65 & $27445_{3}^{\circ}-49223_{3}$ & -6 \\
\hline 4589.211 & 1000 & $150 Z$ & 21784.13 & $25270_{3}-47055_{2}^{\circ}$ & -1 \\
\hline 4585.916 & 40 & $4 Z$ & 21799.79 & & \\
\hline 4585.634 & 15 & & 21801.13 & $27445_{3}^{\circ}-49246_{2}$ & -3 \\
\hline 4582.924 & 60 & 6 & 21814.02 & $258595-476734$ & -1 \\
\hline 4582.695 & 50 & 6 & 21815.11 & $27445_{3}^{\circ}-49260_{4}$ & +0 \\
\hline 4582.355 & $6000 R$ & $600 Z$ & 21816.73 & $17992_{1}^{\circ}-39808_{1}$ & +1 \\
\hline 4581.222 & 7 & & 21822.12 & & \\
\hline 4580.724 & 9 & $1 Z$ & 21824.49 & & \\
\hline 4580.649 & 40 & 6 & 21824.85 & & \\
\hline 4577.722 & 10 & & 21838.81 & & \\
\hline 4576.209 & $10000 R$ & $1000 Z$ & 21846.03 & $17992_{1}^{\circ}-39838_{2}$ & -1 \\
\hline 4568.853 & 200 & $50 Z$ & 21881.20 & $24489_{1}-46370_{1}^{\circ}$ & +0 \\
\hline 4567.368 & 700 & $60 Z$ & 21888.31 & $17992_{1}^{\circ}-39880_{2}$ & +6 \\
\hline 4563.95 & 2000 & $200 Z$ & 21904.70 & $19710_{2}^{\circ}-41615_{1}$ & +5 \\
\hline 4558.095 & 2 & & 21932.84 & $23188_{2}^{\circ}-45121_{1}$ & -1 \\
\hline 4555.47 & 3 & & 21945.48 & & \\
\hline 4552.351 & 6 & & 21960.51 & & \\
\hline 4547.788 & 10 & & 21982.55 & & \\
\hline 4546.866 & 30 & 1 & 21987.01 & & \\
\hline 4545.770 & 8 & 1 & 21992.31 & & \\
\hline
\end{tabular}


TABLE 4. Observed and classified lines of $\mathrm{Yb} \mathrm{I}-\mathrm{Continued}$

\begin{tabular}{|c|c|c|c|c|c|}
\hline \multirow{2}{*}{$\begin{array}{l}\text { Wavelength } \\
\text { (air) } \\
\AA\end{array}$} & \multicolumn{2}{|c|}{ Intensity } & \multirow{2}{*}{$\underset{\mathrm{cm}^{-1}}{\text { Wavenumber }}$} & \multirow{2}{*}{ Classification } & \multirow{2}{*}{$\begin{array}{c}\mathrm{O}-\mathrm{C} \\
0.01 \mathrm{~cm}^{-1}\end{array}$} \\
\hline & $\begin{array}{l}\text { Meggers } \\
\text { Lamp }\end{array}$ & $\begin{array}{c}\text { Thomson } \\
\text { Lamp }\end{array}$ & & & \\
\hline 4544.420 & 30 & 1 & 21998.84 & $27445_{3}^{\circ}-494443$ & -4 \\
\hline 4543.680 & 2 & & 22002.42 & & \\
\hline 4541.452 & 1 & & 22013.22 & & \\
\hline 4540.046 & 8 & 2 & 22020.03 & & \\
\hline 4538.678 & 4 & & 22026.67 & & \\
\hline 4536.313 & 2 & & 22038.16 & & \\
\hline 4533.506 & 1000 & $50 Z$ & 22051.80 & $25859_{5}^{\circ}-47911_{5}$ & +0 \\
\hline 4533.006 & 150 & 8 & 22054.23 & $25270_{3}-47325_{3}^{\circ}$ & -6 \\
\hline 4532.702 & 50 & 2 & 22055.71 & $25270_{3}-47326_{2}^{\circ}$ & -4 \\
\hline 4531.333 & 1500 & $200 Z$ & 22062.37 & & \\
\hline 4529.87 & 3000 & $400 Z$ & 22069.50 & $17992_{1}^{\circ}-40061_{2}$ & +0 \\
\hline 4520.918 & 2 & & 22113.20 & & \\
\hline 4520.510 & 5 & 2 & 22115.20 & & \\
\hline 4520.161 & 200 & 2 & 22116.90 & $19710_{2}^{\circ}-41827_{3}$ & -1 \\
\hline 4513.408 & 1000 & $80 Z$ & 22149.99 & $23188_{2}^{\circ}-45338_{2}$ & -2 \\
\hline 4503.636 & 600 & $50 Z$ & 22198.05 & $25859_{5}^{\circ}-48057_{5}$ & -3 \\
\hline 4503.21 & 150 & 5 & 22200.15 & $25270_{3}-47471_{2}^{\circ}$ & -4 \\
\hline 4498.098 & 1 & & 22225.38 & & \\
\hline 4495.840 & 1 & & 22236.55 & & \\
\hline 4492.120 & 4 & & 22254.96 & & \\
\hline 4490.161 & 2 & & 22264.67 & & \\
\hline 4488.282 & 1000 & $60 Z$ & 22273.99 & $23188_{2}^{\circ}-454623$ & -3 \\
\hline 4483.152 & 8 & 1 & 22299.48 & & \\
\hline 4482.422 & 1500 & $150 Z$ & 22303.11 & $24751_{2}-47055_{2}^{\circ}$ & +1 \\
\hline 4478.271 & 30 & 1 & 22323.78 & $27314_{6}^{\circ}-496385$ & -3 \\
\hline 4473.005 & 20 & 2 & 22350.06 & $30524_{5}^{\circ}-528746$ & -1 \\
\hline 4472.470 & 1500 & $300 Z$ & 22352.74 & $25068_{1}^{\circ}-47420_{2}$ & +0 \\
\hline 4468.520 & 4 & & 22372.50 & & \\
\hline 4468.18 & 10 & 1 & 22374.20 & & \\
\hline 4461.72 & 7 & & 22406.59 & $23188_{2}^{\circ}-455951$ & -3 \\
\hline 4455.651 & 4 & 1 & 22437.11 & & \\
\hline 4441.031 & 9 & 2 & 22510.97 & & \\
\hline 4440.011 & 4 & & 22516.15 & $25068_{1}^{\circ}-47584_{1}$ & +3 \\
\hline 4439.19 & $7000 R$ & $500 Z$ & 22520.31 & $17288^{\circ}-398081$ & +3 \\
\hline 4436.366 & 4 & & 22534.65 & & \\
\hline 4434.795 & 20 & 1 & 22542.63 & & \\
\hline 4432.041 & 1 & & 22556.64 & & \\
\hline 4430.208 & 900 & $150 Z$ & 22565.97 & $24489_{1}-47055_{2}^{\circ}$ & +2 \\
\hline 4428.980 & 30 & 1 & 22572.22 & $25270_{3}-47843_{3}^{\circ}$ & +0 \\
\hline 4428.782 & 700 & 100 & 22573.23 & $24751_{2}-47325_{3}^{\circ}$ & -1 \\
\hline 4428.02 & 3 & & 22577.12 & $250681-47645_{1}$ & -6 \\
\hline 4427.387 & 600 & 200 & 22580.35 & $24751_{2}-47332_{1}^{\circ}$ & +3 \\
\hline 4422.070 & 1 & & 22607.50 & & \\
\hline 4419.616 & 3 & & 22620.05 & & \\
\hline 4415.202 & 1 & & 22642.66 & & \\
\hline
\end{tabular}


TABLE 4. Observed and classified lines of $\mathrm{Yb} \mathrm{I}$-Continued

\begin{tabular}{|c|c|c|c|c|c|}
\hline \multirow{2}{*}{$\begin{array}{l}\text { Wavelength } \\
\text { (air) } \\
\AA\end{array}$} & \multicolumn{2}{|c|}{ Intensity } & \multirow{2}{*}{$\underset{\mathrm{cm}^{-1}}{\text { Wavenumber }}$} & \multirow{2}{*}{ Classification } & \multirow{2}{*}{$\mid \begin{array}{c}\mathrm{O}-\mathrm{C} \\
0.01 \mathrm{~cm}^{-1}\end{array}$} \\
\hline & $\begin{array}{c}\text { Meggers } \\
\text { Lamp }\end{array}$ & $\begin{array}{c}\text { Thomson } \\
\text { Lamp }\end{array}$ & & & \\
\hline 4411.095 & 500 & $30 Z$ & 22663.74 & & \\
\hline 4410.230 & 200 & 8 & 22668.19 & $25270_{3}-47939_{4}^{\circ}$ & -1 \\
\hline 4407.081 & 5 & 1 & 22684.39 & & \\
\hline 4402.605 & 40 & $2 Z$ & 22707.45 & & \\
\hline 4400.332 & 50 & 2 & 22719.18 & $24751_{2}-47471_{2}^{\circ}$ & +2 \\
\hline 4398.96 & 2000 & $100 Z$ & 22726.26 & $19710_{2}^{\circ}-424361$ & -5 \\
\hline 4396.254 & 100 & $5 Z$ & 22740.25 & & \\
\hline 4395.52 & 1 & & 22744.05 & & \\
\hline 4394.973 & 70 & 3 & 22746.88 & $24751_{2}-47498_{1}^{\circ}$ & +0 \\
\hline 4394.177 & 25 & 2 & 22751.00 & & \\
\hline 4393.688 & 2000 & $400 h Z$ & 22753.53 & $25068_{1}^{\circ}-47821_{2}$ & -2 \\
\hline 4387.32 & 30 & 2 & 22786.56 & & \\
\hline 4386.50 & 200 & 5 & 22790.81 & & \\
\hline 4385.14 & 100 & 2 & 22797.88 & & \\
\hline 4384.29 & 3 & & 22802.30 & & \\
\hline 4381.87 & 2 & 1 & 22814.90 & & \\
\hline 4379.452 & 200 & 20 & 22827.49 & & \\
\hline 4379.268 & 60 & 3 & 22828.45 & $25859_{5}^{\circ}-486886$ & -5 \\
\hline 4377.527 & 3000 & $300 h l$ & 22837.53 & $24489_{1}-47326_{2}^{\circ}$ & -2 \\
\hline 4376.456 & 2000 & $200 Z$ & 22843.12 & $24489_{1}-47332^{\circ}$ & -4 \\
\hline 4373.116 & 20 & 1 & 22860.57 & & \\
\hline 4368.679 & 100 & $5 h$ & 22883.78 & $25270_{3}-48154_{3}^{\circ}$ & -2 \\
\hline 4368.23 & 10 & & 22886.14 & & \\
\hline 4366.284 & 1000 & $200 h l$ & 22896.34 & & \\
\hline 4363.71 & 3 & & 22909.84 & & \\
\hline 4361.639 & 100 & $7 h$ & 22920.72 & $244891-47409_{0}^{\circ}$ & +0 \\
\hline 4359.528 & 1000 & $150 h Z$ & 22931.82 & $24489_{1}-47420_{1}^{\circ}$ & -1 \\
\hline 4359.165 & 3 & 2 & 22933.73 & & \\
\hline 4356.676 & 500 & 40 & 22946.83 & $258595-488066$ & -6 \\
\hline 4354.037 & 2 & 1 & 22960.74 & & \\
\hline 4353.570 & 500 & $90 h l$ & 22963.20 & $25270_{3}-48234_{2}^{\circ}$ & -2 \\
\hline 4352.948 & 2000 & $400 Z$ & 22966.48 & $25270_{3}-48237_{3}^{\circ}$ & -1 \\
\hline 4351.67 & 1 & & 22973.23 & & \\
\hline 4350.006 & 40 & 1 & 22982.01 & $24489_{1}-47471_{2}^{\circ}$ & +2 \\
\hline 4349.470 & 2 & 1 & 22984.85 & & \\
\hline 4347.66 & 3 & & 22994.42 & & \\
\hline 4344.762 & 300 & 20 & 23009.75 & $24489_{1}-47498_{1}^{\circ}$ & +2 \\
\hline 4343.632 & 3 & & 23015.74 & & \\
\hline 4343.110 & 2 & & 23018.50 & & \\
\hline 4339.71 & 4 & & 23036.54 & & \\
\hline 4339.38 & 30 & & 23038.29 & & \\
\hline 4337.599 & 80 & 20 & 23047.75 & & \\
\hline 4336.430 & 1000 & $150 h l$ & 23053.96 & $25270_{3}-48324_{2}^{\circ}$ & -3 \\
\hline 4335.360 & 20 & 1 & 23059.65 & & \\
\hline 4333.909 & 400 & 40 & 23067.37 & $25068_{1}^{\circ}-48135_{2}$ & +0 \\
\hline
\end{tabular}


TABLE 4. Observed and classified lines of $\mathrm{Yb} \mathrm{I}-$ Continued

\begin{tabular}{|c|c|c|c|c|c|}
\hline \multirow{2}{*}{$\begin{array}{c}\text { Wavelength } \\
\text { (air) } \\
\AA\end{array}$} & \multicolumn{2}{|c|}{ Intensity } & \multirow{2}{*}{$\underset{\substack{\text { Wavenumber } \\
\mathrm{cm}^{-1}}}{\text { Wat }}$} & \multirow[b]{2}{*}{ Classification } & \multirow{2}{*}{$\begin{array}{c}\mathrm{O}-\mathrm{C} \\
0.01 \mathrm{~cm}^{-1}\end{array}$} \\
\hline & $\begin{array}{l}\text { Meggers } \\
\text { Lamp }\end{array}$ & $\begin{array}{l}\text { Thomson } \\
\text { Lamp }\end{array}$ & & & \\
\hline 4330.488 & 20 & 1 & 23085.59 & & \\
\hline 4329.718 & 70 & $4 Z$ & 23089.70 & & \\
\hline 4326.404 & 2000 & $200 Z$ & 23107.39 & $24751_{2}-47859_{1}^{\circ}$ & +2 \\
\hline 4313.567 & 30 & 1 & 23176.15 & & \\
\hline 4313.156 & 70 & 4 & 23178.36 & $25270_{3}-48449_{2}^{\circ}$ & -1 \\
\hline 4312.991 & 60 & 3 & 23179.25 & & \\
\hline 4312.356 & 600 & 40 & 23182.66 & & \\
\hline 4311.86 & 2 & & 23185.33 & & \\
\hline 4309.823 & 3000 & $300 Z$ & 23196.28 & & \\
\hline 4307.820 & 300 & 10 & 23207.07 & $23188_{2}^{\circ}-46395_{3}$ & -1 \\
\hline 4305.966 & 5000 & $2000 Z$ & 23217.06 & $25270_{3}-48487_{3}^{\circ}$ & -2 \\
\hline 4305.484 & 150 & 20 & 23219.66 & $25859_{5}^{\circ}-49079_{5}$ & +0 \\
\hline 4300.984 & 1000 & $80 Z$ & 23243.95 & $258595-491034$ & +0 \\
\hline 4295.88 & 3 & & 23271.57 & & \\
\hline 4295.026 & 500 & $50 h s$ & 23276.20 & & \\
\hline 4294.473 & 30 & 2 & 23279.19 & $23188_{2}^{\circ}-46467_{2}$ & +1 \\
\hline 4292.99 & 3 & & 23287.24 & & \\
\hline 4292.615 & 10 & 1 & 23289.27 & $25068_{1}^{\circ}-48357_{1}$ & -4 \\
\hline 4292.083 & 70 & 2 & 23292.16 & $\left\{\begin{array}{l}25068_{1}^{\circ}-48360_{1} \\
23188_{2}^{\circ}-46480_{3}\end{array}\right.$ & $\left\{\begin{array}{l}-5 \\
+3\end{array}\right.$ \\
\hline 4291.954 & 3 & 2 & 23292.86 & & \\
\hline 4289.605 & 1 & & 23305.61 & & \\
\hline 4288.840 & 80 & 7 & 23309.77 & & \\
\hline 4284.170 & 2000 & $500 h Z$ & 23335.18 & & \\
\hline 4282.098 & 40 & 2 & 23346.47 & & \\
\hline 4281.850 & 20 & 1 & 23347.82 & & \\
\hline 4277.738 & 4000 & $1000 Z$ & 23370.26 & $24489_{1}-47859_{1}^{\circ}$ & +6 \\
\hline 4275.66 & 5 & & 23381.62 & & \\
\hline 4275.48 & 2 & & 23382.61 & & \\
\hline 4273.74 & 10 & 1 & 23392.13 & & \\
\hline 4272.647 & 400 & $40 h l$ & 23398.11 & & \\
\hline 4272.103 & 1000 & 40 & 23401.09 & $25859_{5}^{\circ}-49260_{4}$ & +2 \\
\hline 4271.798 & 600 & $70 h l$ & 23402.76 & $24751_{2}-48154_{3}^{\circ}$ & +0 \\
\hline 4268.39 & 1 & & 23421.45 & & \\
\hline 4267.132 & 500 & $30 h l$ & 23428.35 & $48761_{1}^{\circ}-72190_{2}$ & -1 \\
\hline 4266.983 & 300 & 30 & 23429.17 & & \\
\hline 4266.70 & 20 & 1 & 23430.72 & $25270_{3}-48701_{2}^{\circ}$ & +2 \\
\hline 4266.135 & $3 d$ & & 23433.82 & & \\
\hline 4261.346 & 20 & 1 & 23460.16 & $24751_{2}-48212_{1}^{\circ}$ & +1 \\
\hline 4258.743 & 200 & $20 h l$ & 23474.50 & & \\
\hline 4257.550 & 40 & 10 & 23481.08 & & \\
\hline 4257.36 & 60 & 3 & 23482.12 & $24751_{2}-48234_{2}^{\circ}$ & -5 \\
\hline 4256.756 & 1000 & 100 & 23485.46 & $24751_{2}-48237_{3}^{\circ}$ & +2 \\
\hline 4253.363 & 3 & & 23504.19 & & \\
\hline 4253.292 & 1 & & 23504.58 & & \\
\hline
\end{tabular}


TABLE 4. Observed and classified lines of $\mathrm{Yb} \mathrm{I}$-Continued

\begin{tabular}{|c|c|c|c|c|c|}
\hline \multirow{2}{*}{$\begin{array}{c}\text { Wavelength } \\
\text { (air) } \\
\AA\end{array}$} & \multicolumn{2}{|c|}{ Intensity } & \multirow{2}{*}{$\underset{\mathrm{cm}^{-1}}{\text { Wavenumber }}$} & \multirow[b]{2}{*}{ Classification } & \multirow{2}{*}{$\mid \begin{array}{c}\mathrm{O}-\mathrm{C} \\
0.01 \mathrm{~cm}^{-1}\end{array}$} \\
\hline & $\begin{array}{l}\text { Meggers } \\
\text { Lamp }\end{array}$ & $\begin{array}{c}\text { Thomson } \\
\text { Lamp }\end{array}$ & & & \\
\hline 4252.941 & 20 & 1 & 23506.52 & $24751_{2}-48258_{1}^{\circ}$ & +0 \\
\hline 4251.521 & 2000 & $200 Z$ & 23514.37 & $19710_{2}^{\circ}-43224_{2}$ & -2 \\
\hline 4246.88 & 4 & & 23540.07 & & \\
\hline 4245.245 & 10 & 1 & 23549.14 & & \\
\hline 4243.92 & 3 & & 23556.49 & & \\
\hline 4242.86 & 4 & & 23562.37 & & \\
\hline 4240.95 & 6 & & 23572.98 & $24751_{2}-48324_{2}^{\circ}$ & +4 \\
\hline 4238.63 & 2 & 1 & 23585.89 & & \\
\hline 4237.00 & 5 & & 23594.96 & & \\
\hline 4235.69 & 7 & & 23602.26 & & \\
\hline 4235.49 & 2 & & 23603.37 & & \\
\hline 4233.445 & 150 & $8 Z$ & 23614.77 & $40563_{1}^{\circ}-64178_{2}$ & +4 \\
\hline 4231.972 & 10000 & $1000 Z$ & 23622.99 & $17992^{\circ}-41615_{1}$ & -4 \\
\hline 4229.657 & 4 & & 23635.92 & & \\
\hline 4226.152 & 300 & $10 h$ & 23655.52 & & \\
\hline 4223.621 & 50 & 2 & 23669.70 & & \\
\hline 4222.32 & 5 & & 23676.99 & & \\
\hline 4220.507 & 7 & 1 & 23687.16 & & \\
\hline 4219.706 & 500 & $20 h$ & 23691.66 & & \\
\hline 4219.247 & 1000 & 200 & 23694.24 & $252703-48965_{2}^{\circ}$ & -2 \\
\hline 4218.693 & 3000 & $600 Z$ & 23697.35 & $24751_{2}-48449_{2}^{\circ}$ & +3 \\
\hline 4216.766 & 20 & & 23708.18 & & \\
\hline 4215.485 & 2 & & 23715.38 & & \\
\hline 4214.29 & 3 & & 23722.11 & & \\
\hline 4213.962 & $50 d$ & $20 h$ & 23723.95 & & \\
\hline 4213.106 & 10 & 1 & 23728.77 & & \\
\hline 4211.82 & 2000 & $300 Z$ & 23736.02 & $24751_{2}-48487_{3}^{\circ}$ & -1 \\
\hline 4210.48 & 5 & & 23743.57 & & \\
\hline 4210.299 & 200 & $20 Z$ & 23744.59 & & \\
\hline 4210.225 & 15 & & 23745.01 & $24489_{1}-48234_{2}^{\circ}$ & -1 \\
\hline 4209.342 & 8 & 1 & 23749.99 & & \\
\hline 4207.32 & 6 & 1 & 23761.41 & & \\
\hline 4205.907 & 2 & & 23769.39 & $24489_{1}-482581$ & +2 \\
\hline 4205.785 & 80 & $6 h$ & 23770.08 & $25068_{1}^{\circ}-488381$ & -3 \\
\hline 4204.195 & 300 & 5 & 23779.07 & $258595-496385$ & +2 \\
\hline 4202.907 & 6 & 2 & 23786.35 & & \\
\hline 4201.864 & 3 & 1 & 23792.26 & & \\
\hline 4201.291 & 200 & $8 h$ & 23795.50 & & \\
\hline 4200.72 & 60 & 1 & 23798.74 & & \\
\hline 4198.18 & 2 & & 23813.14 & & \\
\hline 4197.876 & 400 & $100 h$ & 23814.86 & $25068_{1}^{\circ}-48883_{2}$ & -2 \\
\hline 4196.54 & 1 & & 23822.44 & & \\
\hline 4195.056 & 2 & & 23830.87 & & \\
\hline 4194.39 & 2 & & 23834.65 & & \\
\hline 4194.21 & $2 d$ & & 23835.68 & $24489_{1}-48324_{2}^{\circ}$ & -11 \\
\hline
\end{tabular}


TABLE 4. Observed and classified lines of $\mathrm{Yb} \mathrm{I}-\mathrm{Continued}$

\begin{tabular}{|c|c|c|c|c|c|}
\hline \multirow{2}{*}{$\begin{array}{c}\text { Wavelength } \\
\text { (air) } \\
\AA\end{array}$} & \multicolumn{2}{|c|}{ Intensity } & \multirow{2}{*}{$\underset{\mathrm{cm}^{-1}}{\text { Wavenumber }}$} & \multirow[b]{2}{*}{ Classification } & \multirow{2}{*}{$\begin{array}{c}\mathrm{O}-\mathrm{C} \\
0.01 \mathrm{~cm}^{-1}\end{array}$} \\
\hline & $\begin{array}{l}\text { Meggers } \\
\text { Lamp }\end{array}$ & $\begin{array}{c}\text { Thomson } \\
\text { Lamp }\end{array}$ & & & \\
\hline 4191.36 & 1 & & 23851.88 & & \\
\hline 4189.15 & 3 & & 23864.47 & & \\
\hline 4188.993 & 70 & $5 h$ & 23865.36 & $48324_{2}^{\circ}-72190_{2}$ & +5 \\
\hline 4187.73 & 1 & & 23872.56 & & \\
\hline 4186.75 & 1 & & 23878.15 & & \\
\hline 4184.289 & 2 & 1 & 23892.19 & & \\
\hline 4183.129 & 5 & & 23898.81 & & \\
\hline 4182.07 & 9 & 1 & 23904.87 & & \\
\hline 4179.95 & 20 & & 23916.99 & & \\
\hline 4175.868 & 100 & 10 & 23940.37 & & \\
\hline 4174.56 & 3000 & $300 Z$ & 23947.87 & $17992_{1}^{\circ}-419390$ & -2 \\
\hline 4174.259 & 80 & $4 h$ & 23949.60 & $24751_{2}-48701_{2}^{\circ}$ & -5 \\
\hline 4173.134 & 9 & 1 & 23956.05 & $48234_{2}^{\circ}-72190_{2}$ & -3 \\
\hline 4172.422 & 200 & 20 & 23960.14 & $24489_{1}-48449_{2}^{\circ}$ & -3 \\
\hline 4171.222 & 20 & 1 & 23967.03 & $24751_{2}-48719_{1}^{\circ}$ & -5 \\
\hline 4169.260 & 50 & $5 h$ & 23978.31 & & \\
\hline 4169.141 & 50 & 5 & 23979.00 & & \\
\hline 4166.743 & 40 & 3 & 23992.80 & $36060_{4}-600535$ & +0 \\
\hline 4163.770 & 3 & & 24009.93 & $24751_{2}-48761_{1}^{\circ}$ & +4 \\
\hline 4163.502 & 10 & 2 & 24011.47 & $25270_{3}-49282_{4}^{\circ}$ & +2 \\
\hline 4160.243 & 4 & & 24030.28 & $438146-67844_{6}^{\circ}$ & +5 \\
\hline 4159.008 & 6 & 1 & 24037.42 & & \\
\hline 4155.385 & 8 & 1 & 24058.37 & & \\
\hline 4155.123 & 50 & $2 h$ & 24059.89 & & \\
\hline 4154.83 & 4 & & 24061.59 & & \\
\hline 4152.290 & 2 & & 24076.31 & & \\
\hline 4152.058 & 3 & & 24077.65 & & \\
\hline 4151.19 & 9 & 1 & 24082.69 & & \\
\hline 4149.066 & $20000 R$ & $2000 Z$ & 24095.02 & $19710_{2}^{\circ}-43805_{1}$ & -2 \\
\hline 4146.870 & 80 & 5 & 24107.77 & & \\
\hline 4143.498 & 500 & $30 h$ & 24127.39 & & \\
\hline 4140.307 & 8 & 1 & 24145.99 & & \\
\hline 4139.397 & 2 & & 24151.30 & & \\
\hline 4139.051 & 500 & 20 & 24153.32 & $23188_{2}^{\circ}-47341_{2}$ & +1 \\
\hline 4134.730 & 20 & 1 & 24178.56 & $25068_{1}^{\circ}-492462$ & -1 \\
\hline 4132.165 & 15 & 2 & 24193.56 & $30524_{5}^{\circ}-547185$ & +3 \\
\hline 4131.255 & 2 & & 24198.89 & & \\
\hline 4130.645 & 2 & & 24202.47 & & \\
\hline 4128.934 & 100 & $4 h$ & 24212.50 & $24489_{1}-48701_{2}^{\circ}$ & +0 \\
\hline 4128.815 & 30 & 2 & 24213.19 & $24751_{2}-48965_{2}^{\circ}$ & -2 \\
\hline 4128.115 & 4 & & 24217.30 & & \\
\hline 4127.170 & 1 & & 24222.84 & & \\
\hline 4125.955 & 3 & & 24229.98 & $24489_{1}-48719_{1}^{\circ}$ & +5 \\
\hline 4125.538 & 150 & $5 h$ & 24232.43 & $23188_{2}^{\circ}-47420_{2}$ & -1 \\
\hline 4125.185 & 20 & 1 & 24234.50 & & \\
\hline
\end{tabular}


TABLE 4. Observed and classified lines of Y b I-Continued

\begin{tabular}{|c|c|c|c|c|c|}
\hline \multirow{2}{*}{$\begin{array}{c}\text { Wavelength } \\
\text { (air) } \\
\AA\end{array}$} & \multicolumn{2}{|c|}{ Intensity } & \multirow{2}{*}{$\underset{\mathrm{cm}^{-1}}{\text { Wavenumber }}$} & \multirow[b]{2}{*}{ Classification } & \multirow{2}{*}{$\begin{array}{c}\mathrm{O}-\mathrm{C} \\
0.01 \mathrm{~cm}^{-1}\end{array}$} \\
\hline & $\begin{array}{l}\text { Meggers } \\
\text { Lamp }\end{array}$ & $\begin{array}{l}\text { Thomson } \\
\text { Lamp }\end{array}$ & & & \\
\hline 4123.75 & 6 & 1 & 24242.93 & & \\
\hline 4122.274 & 4 & 1 & 24251.61 & & \\
\hline 4122.143 & 8 & 1 & 24252.38 & & \\
\hline 4121.902 & 200 & 20 & 24253.80 & $24751_{2}-49005_{1}^{\circ}$ & +1 \\
\hline 4119.25 & 30 & 20 & 24269.42 & & \\
\hline 4119.087 & 30 & 3 & 24270.38 & & \\
\hline 4118.180 & 8 & 2 & 24275.72 & & \\
\hline 4118.07 & 10 & 1 & 24276.37 & & \\
\hline 4116.89 & 1 & & 24283.33 & & \\
\hline 4116.000 & 4 & & 24288.58 & & \\
\hline 4115.70 & 1 & & 24290.35 & & \\
\hline 4114.694 & 1 & & 24296.29 & & \\
\hline 4113.805 & 2 & & 24301.54 & & \\
\hline 4113.389 & 40 & $2 h$ & 24304.00 & & \\
\hline 4111.07 & 8 & & 24317.71 & & \\
\hline 4109.79 & 40 & 2 & 24325.28 & & \\
\hline 4109.574 & 3000 & $400 Z$ & 24326.56 & $17288^{\circ}-41615_{1}$ & -4 \\
\hline 4108.542 & 8 & 1 & 24332.67 & & \\
\hline 4107.222 & 20 & $3 h$ & 24340.49 & & \\
\hline 4104.60 & 2 & & 24356.04 & & \\
\hline 4104.05 & 4 & & 24359.30 & & \\
\hline 4101.335 & 30 & 3 & 24375.43 & $24751_{2}-49127_{1}^{\circ}$ & -1 \\
\hline 4099.338 & 20 & 1 & 24387.30 & & \\
\hline 4098.920 & 4 & & 24389.79 & & \\
\hline 4097.080 & 6 & 2 & 24400.74 & & \\
\hline 4089.908 & 20 & 4 & 24443.53 & & \\
\hline 4089.68 & $20000 R$ & $3000 Z$ & 24444.89 & $17992{ }^{\circ}-424360$ & -1 \\
\hline 4087.67 & 5 & 1 & 24456.91 & $23188_{2}^{\circ}-47645_{1}$ & +3 \\
\hline 4087.480 & $30 d$ & $5 d$ & 24458.05 & $23188_{2}^{\circ}-47646_{3}$ & -5 \\
\hline 4087.343 & 20 & 3 & 24458.87 & & \\
\hline 4084.47 & 4 & & 24476.07 & $24489_{1}-48965_{2}^{\circ}$ & +1 \\
\hline 4083.648 & 15 & $4 h$ & 24481.00 & & \\
\hline 4083.346 & 4 & & 24482.81 & & \\
\hline 4082.992 & 500 & 20 & 24484.93 & $23188_{2}^{\circ}-47673_{3}$ & +0 \\
\hline 4082.586 & 2 & & 24487.37 & & \\
\hline 4078.000 & $10 h$ & & 24514.90 & & \\
\hline 4077.713 & 30 & 10 & 24516.63 & $24489_{1}-49005_{1}^{\circ}$ & -1 \\
\hline 4074.359 & 3 & & 24536.81 & & \\
\hline 4073.846 & 8 & & 24539.90 & $17992{ }_{1}^{\circ}-42531_{2}$ & +4 \\
\hline 4073.288 & 1 & & 24543.26 & & \\
\hline 4072.923 & 2 & & 24545.46 & & \\
\hline 4071.115 & 2 & & 24556.36 & & \\
\hline 4067.461 & 4 & & 24578.42 & & \\
\hline 4067.115 & 30 & 2 & 24580.51 & & \\
\hline 4067.00 & 1 & & 24581.21 & & \\
\hline
\end{tabular}


TABLE 4. Observed and classified lines of $\mathrm{Yb} \mathrm{I}$-Continued

\begin{tabular}{|c|c|c|c|c|c|}
\hline \multirow{2}{*}{$\begin{array}{c}\text { Wavelength } \\
\text { (air) } \\
\AA\end{array}$} & \multicolumn{2}{|c|}{ Intensity } & \multirow{2}{*}{$\underset{\mathrm{cm}^{-1}}{\text { Wavenumber }}$} & \multirow[b]{2}{*}{ Classification } & \multirow{2}{*}{$\begin{array}{c}\mathrm{O}-\mathrm{C} \\
0.01 \mathrm{~cm}^{-1}\end{array}$} \\
\hline & $\begin{array}{l}\text { Meggers } \\
\text { Lamp }\end{array}$ & $\begin{array}{l}\text { Thomson } \\
\text { Lamp }\end{array}$ & & & \\
\hline 4065.963 & $20 h$ & 1 & 24587.48 & & \\
\hline 4063.726 & 1000 & 150 & 24601.01 & $19710_{2}^{\circ}-44311_{1}$ & +2 \\
\hline 4063.455 & 3000 & 500 & 24602.65 & $19710_{2}^{\circ}-44313_{2}$ & -1 \\
\hline 4061.382 & 10 & 1 & 24615.21 & & \\
\hline 4059.470 & 40 & $7 Z$ & 24626.80 & & \\
\hline 4058.625 & 60 & 6 & 24631.93 & & \\
\hline 4058.404 & 150 & 15 & 24633.27 & $231882-478212$ & +1 \\
\hline 4055.24 & 2 & & 24652.49 & & \\
\hline 4052.283 & 5000 & $1000 Z$ & 24670.48 & $19710_{2}^{\circ}-44380_{3}$ & +5 \\
\hline 4052.072 & 1000 & $60 Z$ & 24671.76 & $23188_{2}^{\circ}-47860_{3}$ & +0 \\
\hline 4051.935 & 30 & 3 & 24672.60 & & \\
\hline 4046.37 & 1 & & 24706.53 & & \\
\hline 4044.464 & 3 & 1 & 24718.17 & & \\
\hline 4039.938 & 15 & 1 & 24745.86 & & \\
\hline 4038.98 & 2 & 1 & 24751.73 & & \\
\hline 4036.772 & 20 & 15 & 24765.27 & & \\
\hline 4035.855 & 5 & 3 & 24770.90 & & \\
\hline 4035.51 & 5 & 1 & 24773.01 & & \\
\hline 4021.56 & 2 & 1 & 24858.95 & & \\
\hline 4018.966 & 30 & 3 & 24874.99 & $351785-60053_{5}^{\circ}$ & -1 \\
\hline 4010.825 & 3 & & 24925.48 & & \\
\hline 4007.962 & 10 & 5 & 24943.28 & $297744-547185$ & -1 \\
\hline 4007.356 & 2000 & $500 Z$ & 24947.06 & $23188_{2}^{\circ}-48135_{2}$ & -2 \\
\hline 4006.828 & 15 & 2 & 24950.34 & & \\
\hline 4005.65 & $2 h$ & & 24957.68 & & \\
\hline 4002.04 & $3 h$ & & 24980.19 & & \\
\hline 4000.46 & 10 & & 24990.06 & & \\
\hline 4000.212 & 4 & 1 & 24991.61 & & \\
\hline 3998.879 & 2 & 1 & 24999.94 & & \\
\hline 3998.133 & 3 & & 25004.60 & & \\
\hline 3993.753 & 100 & $10 Z$ & 25032.03 & & \\
\hline 3990.885 & $10000 R$ & $1000 Z$ & 25050.01 & $19710_{2}^{\circ}-44760_{2}$ & +3 \\
\hline 3987.99 & $50000 A$ & $30000 Z$ & 25068.20 & $0 o-25068_{1}^{\circ}$ & -3 \\
\hline 3979.710 & 10 & 2 & 25120.35 & & \\
\hline 3979.095 & 60 & 4 & 25124.24 & $19710_{2}^{\circ}-44834_{1}$ & +1 \\
\hline 3975.283 & 2000 & $100 Z$ & 25148.33 & $17288{ }^{\circ}-424361$ & +7 \\
\hline 3973.87 & 10 & 3 & 25157.27 & & \\
\hline 3972.18 & 3 & & 25167.97 & & \\
\hline 3972.01 & 7 & & 25169.05 & $23188_{2}^{\circ}-48357_{1}$ & +3 \\
\hline 3969.89 & 4 & 1 & 25182.49 & & \\
\hline 3963.07 & 5 & & 25225.83 & & \\
\hline 3961.98 & 70 & $6 Z$ & 25232.77 & $17992{ }_{1}^{\circ}-432242$ & -1 \\
\hline 3961.731 & 5 & 1 & 25234.35 & & \\
\hline 3961.503 & 5 & 1 & 25235.80 & & \\
\hline 3957.270 & 6 & 2 & 25262.80 & & \\
\hline
\end{tabular}


TABLE 4. Observed and classified lines of $\mathrm{Yb} \mathrm{I}-\mathrm{Continued}$

\begin{tabular}{|c|c|c|c|c|c|}
\hline \multirow{2}{*}{$\begin{array}{c}\text { Wavelength } \\
\text { (air) } \\
\AA\end{array}$} & \multicolumn{2}{|c|}{ Intensity } & \multirow{2}{*}{$\begin{array}{c}\text { Wavenumber } \\
\mathrm{cm}^{-1}\end{array}$} & \multirow[b]{2}{*}{ Classification } & \multirow{2}{*}{$\begin{array}{c}\mathrm{O}-\mathrm{C} \\
0.01 \mathrm{~cm}^{-1}\end{array}$} \\
\hline & $\begin{array}{c}\text { Meggers } \\
\text { Ĺamp }\end{array}$ & $\begin{array}{c}\text { Thomson } \\
\text { Lamp }\end{array}$ & & & \\
\hline 3957.05 & 5 & & 25264.20 & & \\
\hline 3943.29 & 4 & 1 & 25352.36 & & \\
\hline 3939.780 & 10 & 1 & 25374.95 & & \\
\hline 3934.182 & $3000 R$ & 200 & 25411.05 & $19710_{2}^{\circ}-45121_{1}$ & +7 \\
\hline 3931.273 & 4 & & 25429.85 & & \\
\hline 3929.30 & 1 & & 25442.62 & & \\
\hline 3926.724 & 30 & 3 & 25459.31 & $23188_{2}^{\circ}-48647_{3}$ & +5 \\
\hline 3924.527 & 10 & 2 & 25473.56 & & \\
\hline 3911.272 & 600 & $60 Z$ & 25559.89 & $27314_{6}^{\circ}-52874_{6}$ & +2 \\
\hline 3910.653 & 20 & 4 & 25563.94 & & \\
\hline 3905.51 & 5 & 1 & 25597.60 & & \\
\hline 3902.386 & $10 h$ & 4 & 25618.09 & & \\
\hline 3901.06 & 10 & 1 & 25626.80 & $38551_{2}^{\circ}-64178_{2}$ & +3 \\
\hline 3900.85 & $4000 R$ & $500 Z$ & 25628.18 & $19710_{2}^{\circ}-45338_{2}$ & +4 \\
\hline 3897.555 & 10 & 2 & 25649.84 & $23188_{2}^{\circ}-48838_{1}$ & +3 \\
\hline 3896.742 & 20 & 3 & 25655.20 & & \\
\hline 3890.767 & 20 & 3 & 25694.59 & $23188_{2}^{\circ}-48883_{2}$ & +0 \\
\hline 3882.068 & 40 & 4 & 25752.17 & $19710_{2}^{\circ}-454623$ & +2 \\
\hline 3875.76 & 1 & & 25794.08 & & \\
\hline 3873.246 & 5 & 1 & 25810.82 & & \\
\hline 3872.852 & $5000 R$ & $1000 Z$ & 25813.45 & $17992_{1}^{\circ}-43805_{1}$ & +4 \\
\hline 3868.907 & 4 & 1 & 25839.77 & & \\
\hline 3865.95 & $3 h$ & & 25859.53 & & \\
\hline 3865.43 & 1 & & 25863.01 & & \\
\hline 3865.14 & 1 & & 25864.95 & & \\
\hline 3864.33 & $20 h$ & & 25870.37 & & \\
\hline 3862.59 & 2 & & 25882.03 & & \\
\hline 3862.46 & 1 & & 25882.90 & & \\
\hline 3862.18 & 10 & 1 & 25884.77 & $19710_{2}^{\circ}-45595_{1}$ & +2 \\
\hline 3860.975 & 2 & & 25892.85 & & \\
\hline 3860.815 & 10 & & 25893.93 & & \\
\hline 3860.64 & 3 & 1 & 25895.10 & & \\
\hline $3856: 00$ & 3 & & 25926.26 & & \\
\hline 3855.883 & 50 & 8 & 25927.04 & & \\
\hline 3854.108 & 2 & & 25938.99 & & \\
\hline 3851.65 & 1 & & 25955.54 & & \\
\hline 3851.36 & 5 & & 25957.49 & & \\
\hline 3850.98 & 7 & & 25960.05 & & \\
\hline 3849.54 & 1 & & 25969.76 & & \\
\hline 3848.792 & 5 & 1 & 25974.81 & & \\
\hline 3845.79 & 1 & & 25995.09 & & \\
\hline 3845.64 & 3 & & 25996.10 & & \\
\hline 3845.35 & 2 & & 25998.06 & & \\
\hline 3843.30 & 2 & & 26011.93 & & \\
\hline 3839.907 & 1500 & $80 Z$ & 26034.91 & $23188_{2}^{\circ}-49223_{3}$ & +8 \\
\hline
\end{tabular}


TABLE 4. Observed and classified lines of $\mathrm{Yb}$ I-Continued

\begin{tabular}{|c|c|c|c|c|c|}
\hline \multirow{2}{*}{$\begin{array}{c}\text { Wavelength } \\
\text { (air) } \\
\AA\end{array}$} & \multicolumn{2}{|c|}{ Intensity } & \multirow{2}{*}{$\underset{\mathrm{cm}^{-1}}{\text { Wavenumber }}$} & \multirow[b]{2}{*}{ Classification } & \multirow{2}{*}{$\begin{array}{c}\mathrm{O}-\mathrm{C} \\
0.01 \mathrm{~cm}^{-1}\end{array}$} \\
\hline & $\begin{array}{l}\text { Meggers } \\
\text { Lamp }\end{array}$ & $\begin{array}{l}\text { Thomson } \\
\text { Lamp }\end{array}$ & & & \\
\hline 3838.287 & 80 & $4 Z$ & 26045.90 & & \\
\hline 3838.02 & 7 & 1 & 26047.71 & & \\
\hline 3837.57 & 5 & 1 & 26050.77 & & \\
\hline 3836.46 & 5 & 1 & 26058.30 & $23188_{2}^{\circ}-49246_{2}$ & +3 \\
\hline 3834.35 & 1 & & 26072.64 & & \\
\hline 3830.905 & 2 & & 26096.09 & & \\
\hline 3828.673 & 20 & 5 & 26111.30 & $46078_{1}^{\circ}-72190_{2}$ & +1 \\
\hline 3826.620 & 5 & 1 & 26125.31 & & \\
\hline 3824.956 & 1 & & 26136.68 & & \\
\hline 3823.241 & 5 & 1 & 26148.40 & & \\
\hline 3817.09 & 1 & & 26190.53 & & \\
\hline 3815.203 & 200 & 10 & 26203.49 & $19710_{2}^{\circ}-45913_{2}$ & +2 \\
\hline 3808.809 & 10 & 1 & 26247.48 & $36060_{4}-623085$ & +0 \\
\hline 3806.73 & 2 & & 26261.81 & & \\
\hline 3801.386 & 2 & & 26298.73 & & \\
\hline 3801.263 & 10 & 1 & 26299.58 & & \\
\hline 3798.402 & 4000 & 400 & 26319.39 & $17992_{1}^{\circ}-44311_{1}$ & +2 \\
\hline 3798.162 & 3000 & 300 & 26321.05 & $17992_{1}^{\circ}-44313_{2}$ & +1 \\
\hline 3796.495 & 2 & & 26332.61 & & \\
\hline 3795.785 & 3 & 1 & 26337.53 & & \\
\hline 3791.741 & 7000 & $600 Z$ & 26365.62 & $17992_{1}^{\circ}-44357_{2}$ & +3 \\
\hline 3782.80 & 1 & & 26427.94 & & \\
\hline 3781.415 & 1 & & 26437.62 & & \\
\hline 3774.323 & 700 & $40 Z$ & 26487.29 & & \\
\hline 3770.095 & $2000 R$ & $500 Z$ & 26517.00 & $17288^{\circ}-43805_{1}$ & +2 \\
\hline 3767.714 & 8 & 2 & 26533.75 & $28184_{4}^{\circ}-547185$ & +2 \\
\hline 3764.59 & 1 & & 26555.77 & & \\
\hline 3762.93 & 1 & & 26567.49 & & \\
\hline 3760.481 & 200 & 6 & 26584.79 & & \\
\hline 3751.62 & 1 & & 26647.58 & & \\
\hline 3751.13 & 1 & & 26651.06 & & \\
\hline 3748.55 & 1 & & 26669.40 & & \\
\hline 3746.33 & 3 & & 26685.20 & $19710_{2}^{\circ}-46395_{3}$ & -1 \\
\hline 3744.943 & 40 & 5 & 26695.09 & & \\
\hline 3744.09 & 2 & & 26701.17 & & \\
\hline 3741.296 & 50 & 6 & 26721.11 & $19710_{2}^{\circ}-46431_{2}$ & +1 \\
\hline 3739.411 & 30 & 3 & 26734.58 & $19710_{2}^{\circ}-46444_{1}$ & +1 \\
\hline 3737.80 & 2 & & 26746.10 & & \\
\hline 3736.901 & 10 & 10 & 26752.53 & & \\
\hline 3736.237 & 400 & $100 Z$ & 26757.29 & $19710_{2}^{\circ}-46467_{2}$ & -2 \\
\hline 3734.694 & $3000 R$ & $300 Z$ & 26768.34 & $17992_{1}^{\circ}-44760_{2}$ & -2 \\
\hline 3734.435 & 1000 & $100 Z$ & 26770.20 & $19710_{2}^{\circ}-46480_{3}$ & -6 \\
\hline 3724.36 & 200 & & 26842.62 & $17992_{1}^{\circ}-448341$ & +1 \\
\hline 3717.28 & 3 & & 26893.74 & & \\
\hline 3717.23 & 1 & & 26894.10 & & \\
\hline
\end{tabular}


TABLE 4. Observed and classified lines of $\mathrm{Yb} \mathrm{I}-$ Continued

\begin{tabular}{|c|c|c|c|c|c|}
\hline \multirow{2}{*}{$\begin{array}{c}\text { Wavelength } \\
\text { (air) } \\
\AA\end{array}$} & \multicolumn{2}{|c|}{ Intensity } & \multirow{2}{*}{$\underset{\mathrm{cm}^{-1}}{\text { Wavenumber }}$} & \multirow[b]{2}{*}{ Classification } & \multirow{2}{*}{$\begin{array}{c}\mathrm{O}-\mathrm{C} \\
0.01 \mathrm{~cm}^{-1}\end{array}$} \\
\hline & $\begin{array}{l}\text { Meggers } \\
\text { Lamp }\end{array}$ & $\begin{array}{c}\text { Thomson } \\
\text { Lamp }\end{array}$ & & & \\
\hline 3715.409 & 50 & 4 & 26907.28 & $30524_{5}^{\circ}-574326$ & -5 \\
\hline 3712.666 & 10 & & 26927.16 & & \\
\hline 3711.95 & 1 & & 26932.36 & & \\
\hline 3706.023 & 10 & $5 Z$ & 26975.43 & & \\
\hline 3705.745 & 1 & & 26977.45 & & \\
\hline 3700.580 & 1000 & $40 Z$ & 27015.10 & $25859_{5}^{\circ}-528746$ & +0 \\
\hline 3699.514 & 3000 & 100 & 27022.89 & $17288^{\circ}-44311_{1}$ & -5 \\
\hline 3698.81 & 2 & & 27028.03 & & \\
\hline 3691.67 & 10 & 5 & 27080.30 & & \\
\hline 3688.81 & 10 & & 27101.30 & & \\
\hline 3688.438 & 10 & 1 & 27104.03 & & \\
\hline 3684.997 & 500 & $40 Z$ & 27129.34 & $17992_{1}^{\circ}-45121_{1}$ & -2 \\
\hline 3679.932 & 200 & 20 & 27166.68 & $19710_{2}^{\circ}-46877_{1}$ & -3 \\
\hline 3677.81 & 2 & & 27182.35 & & \\
\hline 3677.25 & 2 & & 27186.49 & & \\
\hline 3675.720 & 20 & & 27197.81 & & \\
\hline 3666.62 & 200 & $4 Z$ & 27265.31 & & \\
\hline 3659.427 & 4 & & 27318.90 & & \\
\hline 3658.78 & 1 & & 27323.73 & & \\
\hline 3657.233 & $3 h$ & & 27335.29 & & \\
\hline 3656.312 & 10 & 2 & 27342.17 & & \\
\hline 3655.729 & $2000 R$ & $100 Z$ & 27346.53 & $17992_{1}^{\circ}-45338_{2}$ & +1 \\
\hline 3648.150 & 150 & 15 & 27403.34 & $27314_{6}^{\circ}-547185$ & +1 \\
\hline 3647.25 & 1 & & 27410.11 & & \\
\hline 3639.445 & 20 & 8 & 27468.89 & & \\
\hline 3634.525 & 30 & 20 & 27506.07 & & \\
\hline 3630.975 & 4 & & 27532.96 & & \\
\hline 3629.58 & 3 & & 27543.54 & & \\
\hline 3629.233 & 800 & $15 Z$ & 27546.18 & $17288^{\circ}-44834_{1}$ & +1 \\
\hline 3624.109 & 10 & 1 & 27585.12 & & \\
\hline 3621.743 & 400 & 10 & 27603.14 & $17992_{1}^{\circ}-45595_{1}$ & +1 \\
\hline 3620.65 & 2 & & 27611.48 & $360604-63672 \stackrel{\circ}{\circ}$ & -2 \\
\hline 3614.994 & 50 & $7 Z$ & 27654.68 & $29774_{4}^{\circ}-574295$ & -5 \\
\hline 3611.994 & 20 & 1 & 27677.65 & & \\
\hline 3608.356 & 3 & & 27705.55 & $29774_{4}^{\circ}-57480_{5}$ & -3 \\
\hline 3607.703 & 700 & 70 & 27710.56 & $19710_{2}^{\circ}-47420_{2}$ & -1 \\
\hline 3598.662 & 9 & 1 & 27780.18 & $322734-600535$ & +0 \\
\hline 3596.416 & 3 & & 27797.53 & $44392{ }^{\circ}-72190_{2}$ & +3 \\
\hline 3594.93 & 1 & & 27809.02 & & \\
\hline 3591.844 & 150 & 15 & 27832.91 & $17288 \circ-45121_{1}$ & -2 \\
\hline 3580.404 & 100 & 10 & 27921.84 & $17992_{1}^{\circ}-459132$ & -1 \\
\hline 3580.125 & 100 & 20 & 27924.01 & $19710_{2}^{\circ}-476342$ & -1 \\
\hline 3578.710 & 10 & 2 & 27935.06 & $19710_{2}^{\circ}-47645_{1}$ & +4 \\
\hline 3578.561 & 600 & $60 Z$ & 27936.22 & $19710_{2}^{\circ}-47646_{3}$ & -1 \\
\hline 3575.125 & 20 & 2 & 27963.07 & $19710_{2}^{\circ}-47673_{3}$ & +1 \\
\hline
\end{tabular}


TABLE 4. Observed and classified lines of $\mathrm{Yb} \mathrm{I}-\mathrm{Continued}$

\begin{tabular}{|c|c|c|c|c|c|}
\hline \multirow{2}{*}{$\begin{array}{c}\text { Wavelength } \\
\text { (air) } \\
\AA\end{array}$} & \multicolumn{2}{|c|}{ Intensity } & \multirow{2}{*}{$\underset{\mathrm{cm}^{-1}}{\text { Wavenumber }}$} & \multirow[b]{2}{*}{ Classification } & \multirow{2}{*}{$\begin{array}{l}\mathrm{O}-\mathrm{C} \\
0.01 \mathrm{~cm}^{-1}\end{array}$} \\
\hline & $\begin{array}{c}\text { Meggers } \\
\text { Lamp }\end{array}$ & $\begin{array}{c}\text { Thomson } \\
\text { Lamp }\end{array}$ & & & \\
\hline 3573.458 & 3 & 3 & 27976.11 & & \\
\hline 3573.247 & 20 & 2 & 27977.76 & & \\
\hline 3572.448 & 50 & & 27984.02 & & \\
\hline 3569.500 & 2 & & 28007.13 & & \\
\hline 3566.905 & 2 & 1 & 28027.51 & & \\
\hline 3559.570 & 10 & & 28085.26 & & \\
\hline 3559.032 & 1000 & $50 Z$ & 28089.50 & $17992^{\circ}-46081 o$ & -3 \\
\hline 3556.263 & 200 & 15 & 28111.38 & $19710_{2}^{\circ}-47821_{2}$ & -2 \\
\hline 3556.013 & 10 & & 28113.35 & & \\
\hline 3551.405 & 1 & & 28149.83 & $19710_{2}^{\circ}-47860_{3}$ & -6 \\
\hline 3548.18 & 50 & 7 & 28175.41 & $19710_{2}^{\circ}-478851$ & -1 \\
\hline 3547.756 & 5 & 1 & 28178.78 & & \\
\hline 3544.162 & 10 & 1 & 28207.35 & $305245-587324$ & +1 \\
\hline 3541.924 & 8 & 1 & 28225.18 & & \\
\hline 3539.33 & 200 & & 28245.86 & & \\
\hline 3536.815 & $3 h$ & & 28265.95 & & \\
\hline 3531.725 & 20 & 1 & 28306.68 & $17288_{0}^{\circ}-455951$ & -2 \\
\hline 3528.075 & 2 & & 28335.97 & & \\
\hline 3523.148 & 3 & 2 & 28375.59 & & \\
\hline 3521.773 & 5 & & 28386.67 & $305245-589115$ & +0 \\
\hline 3519.82 & 2 & & 28402.42 & & \\
\hline 3518.457 & 300 & 20 & 28413.43 & & \\
\hline 3517.001 & 500 & $60 Z$ & 28425.19 & $19710_{2}^{\circ}-48135_{2}$ & -2 \\
\hline 3515.238 & 20 & 2 & 28439.44 & $17992_{1}^{\circ}-46431_{2}$ & -4 \\
\hline 3513.573 & 400 & $30 Z$ & 28452.92 & $17992^{\circ}-464441$ & -3 \\
\hline 3510.764 & 600 & $50 Z$ & 28475.68 & $17992^{\circ}-46467_{2}$ & -1 \\
\hline 3504.737 & 3 & & 28524.65 & $30207^{\circ}-587324$ & -3 \\
\hline 3503.988 & 20 & 7 & 28530.75 & $43659_{1}^{\circ}-72190_{2}$ & -7 \\
\hline 3500.813 & 10 & 5 & 28556.62 & $30524^{\circ}-590814$ & -2 \\
\hline 3495.623 & 10 & 1 & 28599.02 & $19710_{2}^{\circ}-48309_{1}$ & +0 \\
\hline 3491.133 & 2 & & 28635.80 & & \\
\hline 3489.746 & 60 & 15 & 28647.18 & $19710_{2}^{\circ}-48357_{1}$ & +3 \\
\hline 3489.399 & 8 & 1 & 28650.03 & $19710_{2}^{\circ}-48360_{1}$ & -1 \\
\hline 3488.855 & 200 & 1 & 28654.50 & & \\
\hline 3487.971 & 20 & 5 & 28661.76 & & \\
\hline 3487.603 & 2 & & 28664.78 & & \\
\hline 3484.176 & 7 & & 28692.98 & & \\
\hline 3481.68 & 30 & & 28713.55 & & \\
\hline 3470.106 & 10 & 3 & 28809.31 & $19710_{2}^{\circ}-48519_{1}$ & -1 \\
\hline 3467.26 & 10 & & 28832.96 & & \\
\hline 3464.37 & $30000 \mathrm{~A}$ & $2000 Z$ & 28857.01 & $0 \mathrm{o}-28857^{\circ}$ & +0 \\
\hline 3464.190 & 100 & 20 & 28858.51 & $258595-547185$ & -6 \\
\hline 3462.338 & 20 & 15 & 28873.95 & $30207_{3}^{\circ}-59081_{4}$ & -3 \\
\hline 3461.004 & 100 & 9 & 28885.08 & $17992^{\circ}-46877_{1}$ & -2 \\
\hline 3460.269 & $3000 R$ & $60 Z$ & 28891.21 & & \\
\hline
\end{tabular}


TABLE 4. Observed and classified lines of $\mathrm{Yb} \mathrm{I}$-Continued

\begin{tabular}{|c|c|c|c|c|c|}
\hline \multirow{2}{*}{$\begin{array}{c}\text { Wavelength } \\
\text { (air) } \\
\AA\end{array}$} & \multicolumn{2}{|c|}{ Intensity } & \multirow{2}{*}{$\underset{\mathrm{cm}^{-1}}{\text { Wavenumber }}$} & \multirow[b]{2}{*}{ Classification } & \multirow{2}{*}{$\begin{array}{c}\mathrm{O}-\mathrm{C} \\
0.01 \mathrm{~cm}^{-1}\end{array}$} \\
\hline & $\begin{array}{l}\text { Meggers } \\
\text { Lamp }\end{array}$ & $\begin{array}{c}\text { Thomson } \\
\text { Lamp }\end{array}$ & & & \\
\hline 3459.663 & 100 & $10 Z$ & 28896.27 & & \\
\hline 3459.095 & 60 & 3 & 28901.02 & & \\
\hline 3458.391 & 1000 & 20 & 28906.90 & & \\
\hline 3456.525 & 10 & & 28922.50 & & \\
\hline 3454.75 & 10 & & 28937.36 & $19710_{2}^{\circ}-48647_{3}$ & -3 \\
\hline 3452.398 & 300 & $30 Z$ & 28957.08 & $29774_{4}^{\circ}-587324$ & -2 \\
\hline 3451.82 & 2 & & 28961.93 & & \\
\hline 3451.174 & $300 h$ & $1 h$ & 28967.35 & & \\
\hline 3443.587 & 30 & $20 Z$ & 29031.17 & & \\
\hline 3441.07 & 3 & & 29052.40 & & \\
\hline 3437.17 & 5 & 1 & 29085.36 & & \\
\hline 3433.167 & 100 & 10 & 29119.28 & & \\
\hline 3432.143 & 150 & 15 & 29127.96 & $19710_{2}^{\circ}-48838_{1}$ & +2 \\
\hline 3431.140 & 700 & $20 Z$ & 29136.48 & $29774_{4}^{\circ}-589115$ & +5 \\
\hline 3431.107 & $3000 R$ & $80 Z$ & 29136.76 & & \\
\hline 3428.782 & 700 & 30 & 29156.52 & $17288 \circ-464441$ & +0 \\
\hline 3426.878 & 150 & 30 & 29172.71 & $19710_{2}^{\circ}-48883_{2}$ & -1 \\
\hline 3426.044 & $3000 R$ & $100 Z$ & 29179.82 & & \\
\hline 3424.610 & 1 & & 29192.03 & & \\
\hline 3424.295 & 1 & & 29194.72 & & \\
\hline 3420.345 & 150 & 20 & 29228.43 & & \\
\hline 3419.807 & 3 & & 29233.03 & $19710_{2}^{\circ}-48943_{1}$ & +0 \\
\hline 3418.390 & 400 & $50 Z$ & 29245.15 & $28184_{4}^{\circ}-57429_{5}$ & -2 \\
\hline 3417.044 & 250 & 20 & 29256.67 & & \\
\hline 3412.453 & 80 & $40 Z$ & 29296.03 & $28184_{4}^{\circ}-57480_{5}$ & +0 \\
\hline 3411.240 & 10 & 8 & 29306.45 & $29774_{4}^{\circ}-59081_{4}$ & +4 \\
\hline 3408.95 & 2 & & 29326.13 & & \\
\hline 3407.922 & 2 & & 29334.98 & & \\
\hline 3406.200 & 200 & 5 & 29349.81 & $17992_{1}^{\circ}-47341_{2}$ & +0 \\
\hline 3397.037 & 50 & 2 & 29428.97 & $17992_{1}^{\circ}-47420_{2}$ & +2 \\
\hline 3394.519 & 3 & & 29450.80 & & \\
\hline 3393.972 & 30 & 3 & 29455.55 & & \\
\hline 3389.904 & $8 h$ & & 29490.89 & & \\
\hline 3387.505 & $200 r$ & $50 Z$ & 29511.78 & & \\
\hline 3387.370 & 20 & 2 & 29512.96 & $19710_{2}^{\circ}-492233$ & -1 \\
\hline 3385.329 & 2 & & 29530.75 & . & \\
\hline 3384.682 & 200 & 15 & 29536.39 & $19710_{2}^{\circ}-492462$ & -1 \\
\hline 3382.537 & 20 & 20 & 29555.12 & & \\
\hline 3379.386 & 2 & 2 & 29582.68 & & \\
\hline 3378.698 & 50 & 3 & 29588.70 & $17288 \circ-46877_{1}$ & +4 \\
\hline 3378.286 & 60 & 4 & 29592.31 & $17992_{1}^{\circ}-475841$ & -2 \\
\hline 3372.576 & 500 & 40 & 29642.41 & $17992_{1}^{\circ}-476342$ & +1 \\
\hline 3371.325 & 100 & 10 & 29653.41 & $17992_{1}^{\circ}-47645_{1}$ & +2 \\
\hline 3371.090 & 2 & & 29655.48 & & \\
\hline 3367.324 & 1 & & 29688.64 & & \\
\hline
\end{tabular}


TABLE 4. Observed and classified lines of $\mathrm{Yb} \mathrm{I}-$ Continued

\begin{tabular}{|c|c|c|c|c|c|}
\hline \multirow{2}{*}{$\begin{array}{l}\text { Wavelength } \\
\text { (air) } \\
\AA\end{array}$} & \multicolumn{2}{|c|}{ Intensity } & \multirow{2}{*}{$\begin{array}{c}\text { Wavenumber } \\
\mathrm{cm}^{-1}\end{array}$} & \multirow{2}{*}{ Classification } & \multirow{2}{*}{$\mid \begin{array}{c}O-C \\
0.01 \mathrm{~cm}^{-1}\end{array}$} \\
\hline & $\begin{array}{c}\text { Meggers } \\
\text { Lamp }\end{array}$ & $\begin{array}{c}\text { Thomson } \\
\text { Lamp }\end{array}$ & & & \\
\hline 3366.928 & 10 & & 29692.13 & & \\
\hline 3365.181 & 5 & & 29707.55 & & \\
\hline 3362.770 & 1 & & 29728.85 & & \\
\hline 3362.145 & 1 & & 29734.37 & & \\
\hline 3361.927 & 4 & & 29736.30 & & \\
\hline 3355.618 & 1 & & 29792.21 & & \\
\hline 3353.607 & 1 & & 29810.07 & & \\
\hline 3352.880 & 60 & 4 & 29816.54 & & \\
\hline 3351.382 & 6 & & 29829.86 & $17992_{1}^{\circ}-47821_{2}$ & +9 \\
\hline 3351.265 & 20 & 20 & 29830.90 & & \\
\hline 3350.357 & 1 & & 29838.99 & & \\
\hline 3350.209 & 3 & & 29840.31 & & \\
\hline 3349.713 & 1 & & 29844.73 & & \\
\hline 3347.016 & 2 & & 29868.77 & & \\
\hline 3346.504 & 30 & 30 & 29873.34 & & \\
\hline 3345.958 & 2 & & 29878.22 & & \\
\hline 3345.575 & 5 & & 29881.64 & & \\
\hline 3345.012 & 20 & & 29886.67 & & \\
\hline 3344.507 & 1 & & 29891.18 & & \\
\hline 3344.212 & 30 & 3 & 29893.82 & $17992_{1}^{\circ}-47885_{1}$ & +1 \\
\hline 3339.644 & 2 & & 29934.70 & & \\
\hline 3339.075 & $10 h$ & & 29939.80 & & \\
\hline 3336.68 & 20 & 1 & 29961.29 & & \\
\hline 3332.150 & 9 & & 30002.02 & & \\
\hline 3331.95 & 1 & & 30003.82 & & \\
\hline 3329.93 & 4 & & 30022.03 & & \\
\hline 3328.51 & 2 & & 30034.83 & $32273_{4}-623085$ & -3 \\
\hline 3327.195 & 3 & 3 & 30046.70 & & \\
\hline 3319.674 & 5 & & 30114.77 & $27314 \stackrel{\circ}{-574295}$ & +1 \\
\hline 3319.412 & $700 r$ & $50 Z$ & 30117.15 & $273146-574326$ & +2 \\
\hline 3316.496 & 300 & $30 Z$ & 30143.63 & $179921-481352$ & +4 \\
\hline 3314.533 & 20 & 5 & 30161.48 & & \\
\hline 3314.27 & 1 & & 30163.88 & & \\
\hline 3305.252 & 20 & 20 & 30246.17 & & \\
\hline 3302.978 & 3 & & 30266.99 & & \\
\hline 3302.368 & 7 & & 30272.58 & & \\
\hline 3301.481 & 1 & & 30280.72 & & \\
\hline 3299.828 & 200 & $10 Z$ & 30295.89 & $17288^{\circ}-47584_{1}$ & -1 \\
\hline 3299.647 & 2 & & 30297.55 & & \\
\hline 3297.486 & 8 & & 30317.40 & $17992_{1}^{\circ}-48309_{1}$ & +0 \\
\hline 3293.685 & 7 & & 30352.39 & & \\
\hline 3293.19 & 10 & & 30356.95 & $17288 \circ-476451$ & -1 \\
\hline 3292.26 & 20 & & 30365.53 & $17992_{1}^{\circ}-48357_{1}$ & -1 \\
\hline 3291.946 & 20 & & 30368.42 & $17992_{1}^{\circ}-48360_{1}$ & +0 \\
\hline 3282.33 & 3 & & 30457.39 & & \\
\hline
\end{tabular}


TABLE 4. Observed and classified lines of $\mathrm{Yb} \mathrm{I}-\mathrm{Continued}$

\begin{tabular}{|c|c|c|c|c|c|}
\hline \multirow{2}{*}{$\begin{array}{c}\text { Wavelength } \\
\text { (air) } \\
\AA\end{array}$} & \multicolumn{2}{|c|}{ Intensity } & \multirow{2}{*}{$\underset{\mathrm{cm}^{-1}}{\text { Wavenumber }}$} & \multirow[b]{2}{*}{ Classification } & \multirow{2}{*}{$\begin{array}{c}\mathrm{O}-\mathrm{C} \\
0.01 \mathrm{~cm}^{-1}\end{array}$} \\
\hline & $\begin{array}{l}\text { Meggers } \\
\text { Lamp }\end{array}$ & $\begin{array}{l}\text { Thomson } \\
\text { Lamp }\end{array}$ & & & \\
\hline 3278.614 & 50 & 1 & 30491.91 & & \\
\hline 3278.56 & 5 & & 30492.41 & & \\
\hline 3276.218 & 15 & & 30514.21 & & \\
\hline 3274.770 & 6 & & 30527.70 & $17992_{1}^{\circ}-48519_{1}$ & -1 \\
\hline 3272.644 & 30 & & 30547.53 & $28184_{4}^{\circ}-587324$ & -2 \\
\hline 3268.811 & 2 & & 30583.35 & & \\
\hline 3267.890 & 150 & 2 & 30591.97 & & \\
\hline 3267.313 & 10 & & 30597.37 & $172888^{\circ}-47885_{1}$ & +0 \\
\hline 3265.247 & 2 & & 30616.73 & & \\
\hline 3253.545 & 20 & 1 & 30726.84 & $28184_{4}^{\circ}-58911_{5}$ & -4 \\
\hline 3253.023 & 3 & & 30731.77 & & \\
\hline 3244.46 & 2 & & 30812.88 & & \\
\hline 3241.86 & 30 & 1 & 30837.59 & & \\
\hline 3240.946 & 20 & 1 & 30846.29 & $17992_{1}^{\circ}-488381$ & -4 \\
\hline 3239.580 & 200 & 5 & 30859.29 & $30524 \stackrel{\circ}{-}-61384_{5}$ & +1 \\
\hline 3237.888 & 10 & & 30875.42 & & \\
\hline 3236.24 & 10 & & 30891.14 & $17992_{1}^{\circ}-48883_{2}$ & +4 \\
\hline 3235.635 & 2 & & 30896.92 & $28184_{4}^{\circ}-59081_{4}$ & +7 \\
\hline 3235.520 & 4 & & 30898.01 & & \\
\hline 3229.93 & 2 & & 30951.49 & $17992_{1}^{\circ}-48943_{1}$ & +8 \\
\hline 3227.478 & 100 & 2 & 30975.00 & & \\
\hline 3222.696 & 150 & 3 & 31020.96 & $17288 \circ-48309_{1}$ & -1 \\
\hline 3217.40 & 5 & & 31072.02 & $17288 \circ-48360_{1}$ & +3 \\
\hline 3216.66 & 2 & & 31079.17 & & \\
\hline 3207.870 & 2 & & 31164.33 & & \\
\hline 3207.380 & 6 & & 31169.09 & & \\
\hline 3195.349 & 15 & & 31286.44 & $27445_{3}^{\circ}-587324$ & +2 \\
\hline 3183.90 & 3 & & 31398.94 & $322734-636725$ & +6 \\
\hline 3183.07 & 2 & & 31407.13 & & \\
\hline 3182.09 & 1 & & 31416.80 & & \\
\hline 3169.558 & 10 & & 31541.01 & & \\
\hline 3166.644 & 10 & & 31570.04 & $258595-574295$ & +4 \\
\hline 3166.407 & 10 & & 31572.40 & $258595-574326$ & +3 \\
\hline 3163.985 & 4 & & 31596.57 & $273146-589115$ & +10 \\
\hline 3162.737 & 4 & & 31609.03 & $29774_{4}^{\circ}-613845$ & -1 \\
\hline 3162.293 & 50 & 5 & 31613.47 & & \\
\hline 3161.55 & 2 & & 31620.90 & $25859_{5}^{\circ}-57480_{5}$ & +4 \\
\hline 3158.145 & 1 & & 31654.99 & $17288 \circ-489431$ & +1 \\
\hline 3152.880 & 2 & & 31707.85 & & \\
\hline 3147.450 & 1 & & 31762.55 & & \\
\hline 3130.862 & 2 & & 31930.83 & & \\
\hline 3117.318 & 2 & & 32069.56 & & \\
\hline 3112.742 & 5 & & 32116.70 & & \\
\hline 3100.745 & 5 & & 32240.96 & & \\
\hline 3089.04 & 10 & & 32363.12 & & \\
\hline
\end{tabular}


TABLE 4. Observed and classified lines of $\mathrm{Yb} \mathrm{I}$-Continued

\begin{tabular}{|c|c|c|c|c|c|}
\hline \multirow{2}{*}{$\begin{array}{c}\text { Wavelength } \\
\text { (air) } \\
\AA\end{array}$} & \multicolumn{2}{|c|}{ Intensity } & \multirow{2}{*}{$\underset{\mathrm{cm}^{-1}}{\text { Wavenumber }}$} & \multirow[b]{2}{*}{ Classification } & \multirow{2}{*}{$\begin{array}{c}\mathrm{O}-\mathrm{C} \\
0.01 \mathrm{~cm}^{-1}\end{array}$} \\
\hline & $\begin{array}{l}\text { Meggers } \\
\text { Lamp }\end{array}$ & $\begin{array}{c}\text { Thomson } \\
\text { Lamp }\end{array}$ & & & \\
\hline 3085.21 & 8 & & 32403.29 & & \\
\hline 3081.142 & 2 & & 32446.07 & & \\
\hline 3079.264 & 7 & 1 & 32465.86 & & \\
\hline 3074.63 & 3 & & 32514.79 & & \\
\hline 3074.60 & 2 & & 32515.11 & & \\
\hline 3070.98 & 1 & & 32553.44 & & \\
\hline 3060.440 & 5 & & 32665.54 & $351785-678446$ & -2 \\
\hline 3041.180 & 7 & & 32872.41 & $25859^{\circ}-587324$ & +3 \\
\hline 3027.476 & 7 & & 33021.20 & & \\
\hline 3011.218 & 7 & & 33199.48 & $28184_{4}^{\circ}-61384_{5}$ & -1 \\
\hline 3010.735 & 2 & & 33204.81 & & \\
\hline 3009.21 & 2 & & 33221.63 & $25859 \stackrel{\circ}{5}-59081_{4}$ & -5 \\
\hline 3005.118 & 5 & & 33266.87 & & \\
\hline 3000.865 & 2 & & 33314.01 & & \\
\hline 2952.55 & 4 & & 33859.13 & & \\
\hline 2944.336 & 5 & & 33953.59 & & \\
\hline 2934.360 & 60 & 3 & 34069.02 & $27314_{6}^{\circ}-61384_{5}$ & -6 \\
\hline 2928.62 & 3 & & 34135.79 & & \\
\hline 2927.48 & 3 & & 34149.08 & & \\
\hline 2922.235 & 4 & & 34210.37 & & \\
\hline 2888.24 & 15 & & 34613.01 & & \\
\hline 2883.686 & 10 & 1 & 34667.67 & & \\
\hline 2873.490 & 15 & 1 & 34790.68 & & \\
\hline 2819.24 & 2 & & 35460.11 & & \\
\hline 2814.14 & 2 & & 35524.37 & $25859_{5}^{\circ}-613845$ & +6 \\
\hline 2789.665 & 3 & & 35836.03 & & \\
\hline 2785.903 & 2 & & 35884.42 & & \\
\hline 2784.966 & 4 & & 35896.49 & & \\
\hline 2671.958 & $1000 \mathrm{~A}$ & $10 Z$ & 37414.62 & $0_{0}-37414^{\circ}$ & +3 \\
\hline 2652.28 & 2 & & 37692.19 & & \\
\hline 2651.68 & 2 & & 37700.72 & & \\
\hline 2618.800 & 40 & 1 & 38174.04 & $0_{0}-38174^{\circ}$ & -14 \\
\hline 2601.87 & 20 & 1 & 38422.41 & $0 \mathrm{o}-38422^{\circ}$ & +5 \\
\hline 2580.36 & 3 & & 38742.68 & & \\
\hline 2516.118 & 1 & & 39731.80 & & \\
\hline 2464.50 & $1000 \mathrm{~A}$ & 10 & 40563.91 & $00-40563^{\circ}$ & -6 \\
\hline 2324.44 & 30 & 9 & 43007.91 & & \\
\hline 2289.76 & 4 & & 43659.24 & $0 \mathrm{o}-43659_{\mathrm{i}}$ & -14 \\
\hline 2288.04 & 1 & & 43692.05 & & \\
\hline 2271.11 & 40 & 2 & 44017.73 & $0 o-44017^{\circ}$ & +13 \\
\hline 2212.60 & 3 & & 45181.62 & $0 \mathrm{o}-45181_{1}^{\circ}$ & -7 \\
\hline 2155.88 & 3 & & 46370.19 & $0 \mathrm{o}-46370_{\mathrm{i}}^{\circ}$ & -11 \\
\hline
\end{tabular}

